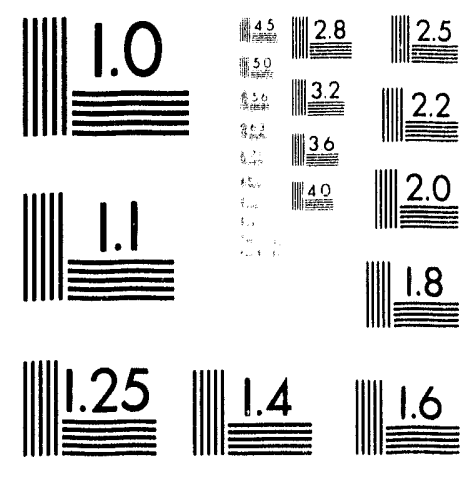



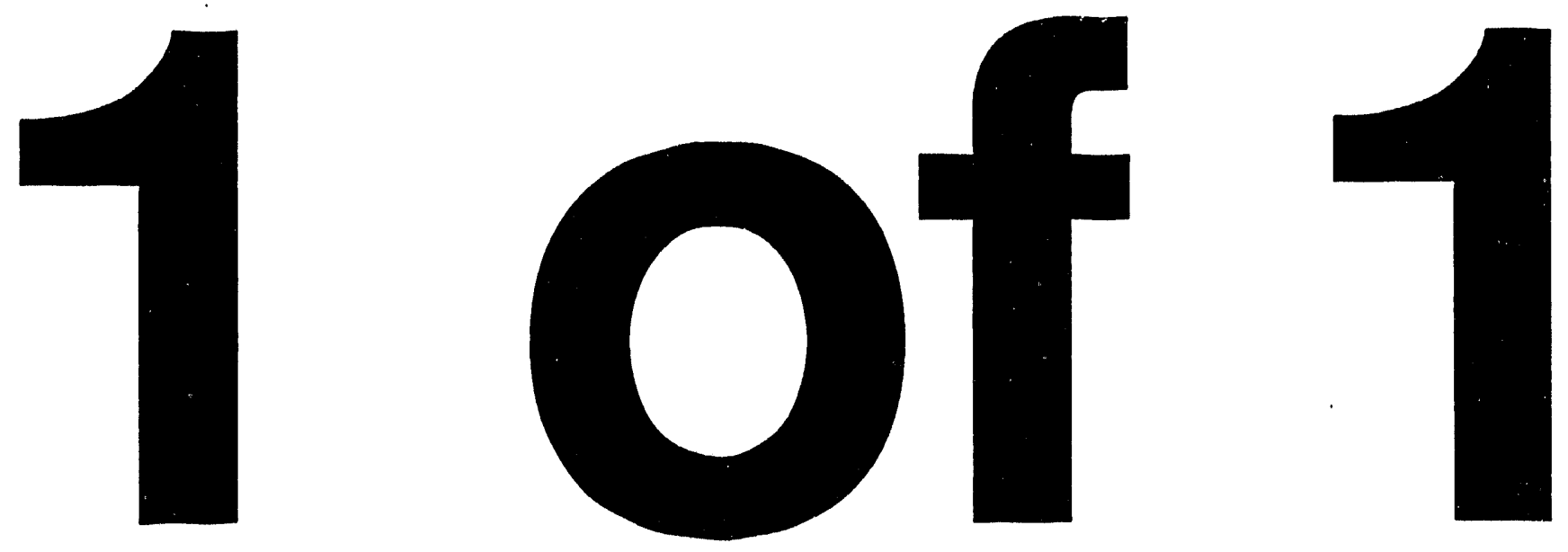


\title{
Superconducting Energy Storage
}

\author{
by R.F. Giese
}

Argonne National Laboratory, 9700 South Cass Avenue, Argonne, Illinois 60439 United States of America

\section{DISCLAIMER}

\begin{abstract}
This report was prepared as an account of work sponsored by an agency of the United States Government. Neither the United States Gnvernment nor any agency thereof, nor any of their employees, makes any warranty, express or implied, or assumes any legal liability or responsibility for the accuracy, completeness, or usefulness of any information, apparatus, product, or process disclosed, or represents that its use would not infringe privately owned rights. Reference herein to any specific commercial product, process, or service by trade name, trademark, manufacturer, or otherwise does not necessarily constitute or imply its endorsement, recommendation, or favoring by the United States Government or any agency thereof. The views and opinions of authors expressed herein do not necessarily state or reflect those of the United States Government or any agency thereof.
\end{abstract}

October 1,1993

Work done for and sponsored by the participating signatories of the International Energy Agency

Implementing Agreement for a Co-Operative Programme for Assessing the Impacts

of High-Temperature Superconductivity on the Electric Power Sector

DISTRIBUTION OF THIS DOCUMENT is IINLIMTE $\mathrm{c}_{2}$ 


\section{Preface}

\section{Content and Purpose}

This report describes the status of energy storage involving superconductors and assesses what impact the recently discovered ceramic superconductors may have on the design of these devices. Our description is intended for R\&D managers in government, electric utilities, firms, and national laboratories who wish an overview of what has been done and what remains to be done. It is assumed that the reader is acquainted with superconductivity, but not an expert on the topics discussed here. Indeed, it is the author's aim to enable the reader to better understand the experts who may ask for the reader's attention, support, or funding. This report may also inform scientists and engineers who, though expert in related areas, wish to have an introduction to our topic.

\section{Sponsorship}

The effort to write this report was sponsored by institutions in Canada, Denmark, Finland, Germany, Israel, Italy, Japan, the Netherlands, Norway, Sweden, Switzerland, Turkey, the United Kingdom, and the United States. These institutions are signatories to an International Energy Agency (IEA) Implementing Agreement, entitled Implementing Agreement for a Co-Operative Programme for Assessing the Impacts of High-Temperature Superconductivity on the Electric Power Sector.

The names and addresses of representative to the sponsors' Executive Committee for the project from participating countries are given on the next pages.

\section{Further Sources of Information}

Further information about ongoing efforts on superconducting energy storage can be gained by contacting the researchers engaged in those efforts. Access to them can be facilitated by referring to the "Directory of Ceramic Supercondurtor Research and Related Research Bearing upon the Electric Power Sector", another report prepared by Argonne National Laboratory for the participating signatories of the International Energy Agency Implementing Agreement for a Co-Operative Programme for Assessing the Impacts of High-Temperature Superconductivity on the Electric Power Sector. Copies of that report can be obtained by requesting it from the member of the sponsors' Executive Committee who represents the country of the requester. The name and address of each member of the Executive Committee is listed on the next pages. 
Dr. F.Y.Chu

Section Head

Science Research Division

Ontario Hydro

800 Kipling Avenue

Toronto, Ontario

M8Z5S4, CANADA

Dr. Raymond Roberge

Hydro-Quebec

1800 Montée Ste. Julie

Varennes, Quebec

J3X151

CANADA

Mr. Frank Rasmussen

Head, Electrical Section of Power System

Evaluation Dept.

Elkraft Power Co., Ltd.

Lautruphoej 5

2750 Ballerup

DENMARK

Prof. Jarl-Thure Eriksson

Professor

Electrotechnical Department

Tampere University of Technology

P.O. Box 527

SF-33101 Tampere 10

FINLAND

Mr. Jeffrey Skeer

Office of Research, Development and

Technology Applications

International Energy Agency

2 rue André-Pascal

F-75775 Paris CEDEX 16

Paris, FRANCE

Prof. Dr. Peter Komarek

Director of Institute for Technical Physics

Nuclear Research Centre

W-7500 Karlsruhe

P.O. Box 3640

GERMANY
Prof. Guy Deutscher

Tel Aviv University

Ramat Aviv 69978

Tel Aviv

ISRAEL

Dr. Gabriele Botta

ENEL-CREL

Via Volta 1

20093 Cologno Monzese

Milano, ITALY

Dr. Toshimichi Ishimaru

Director General

Superconductivity Project Team

New Energy and Industrial Technology

Development Organization (NEDO)

Sunshine 60, 1-1 Higashi-Ikebukuro

3-chrome

Toshima-ku

Tokyo 170

JAPAN

Dr. Fred Steennis

Group Leader, Power Applications

KEMA

P.O. Box 9035

6800 Arnheim

NETHERLANDS

Dr. John Kulsetás

Research Director

Norwegian Electric Power Research Institute (EFI)

7034 Trondheim

NORWAY

Dr. Ingela Agrell

National Energy Administration

Liljeholmsvägam 30

S-11787 Stockholm

SWEDEN 
Geog Vécsey

Head of the Technical Physics Laboratory

Paul Scherrer Institute (PSI)

Wurlenlingen and Villingen

CH-5232 Villigen PSI

SWITZERLAND

Mr. Ömer Kaymakcalan

President

Marmara Research Center

Centre Tübitak

P.O. Box 21

41470 Gebze-Kocaeli

TURKEY
Mr. David Rose

Manufacturing Technology Division

The Department for Enterprise

Department of Trade and Industry

151 Buckingham Palace Road

London SWIW9SS

UNITED KINGDOM

Dr. James G. Daley

U.S. Department of Energy

Conservation and Renewable Energy

CE-142 5E-036

1000 Independence Ave., SW

Washington, DC 20585

UNITED STATES 


\section{Contents}

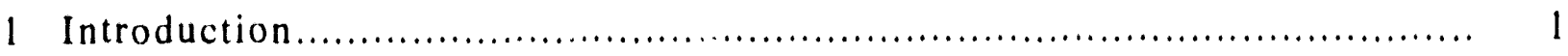

2 Superconducting Energy Storage........................................ 3

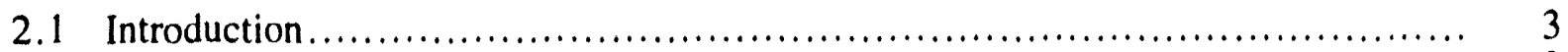

2.2 Superconducting Magnetic Energy Storage................................ 3

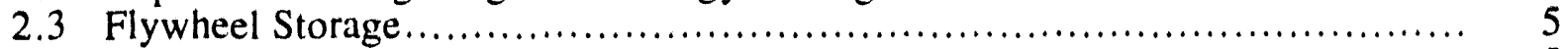

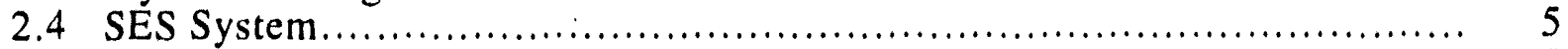

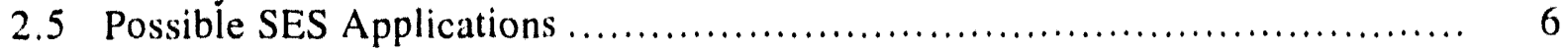

3 Design Tradeoffs for SMES ............................................. 9

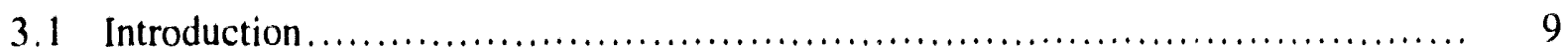

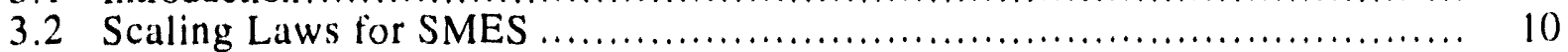

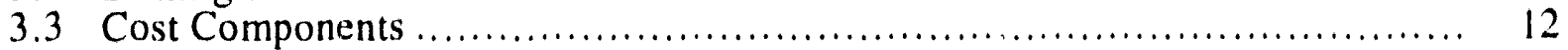

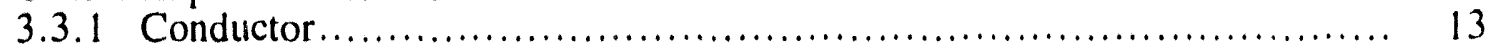

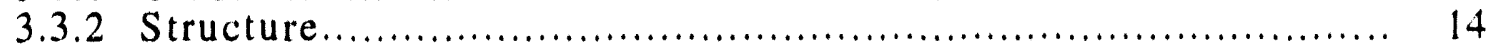

3.3.3 Power Conditioning System .................................... 15

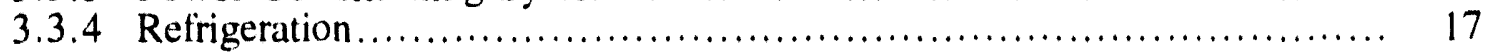

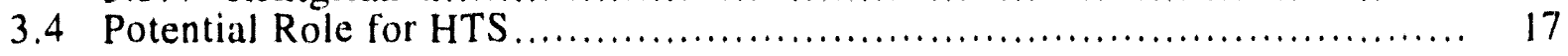

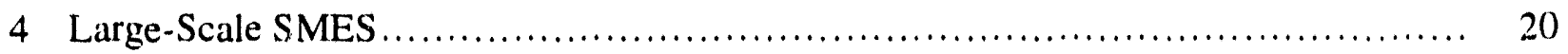

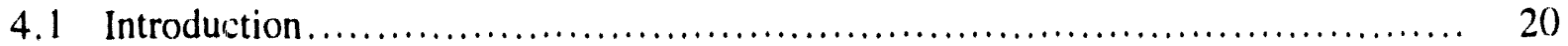

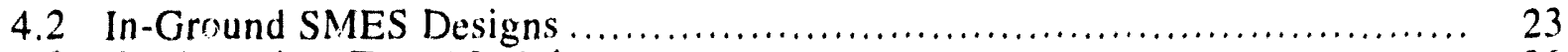

4.3 Engineering Test Model............................................... 26

5 Smail-Scale SMES ..................................................... 30

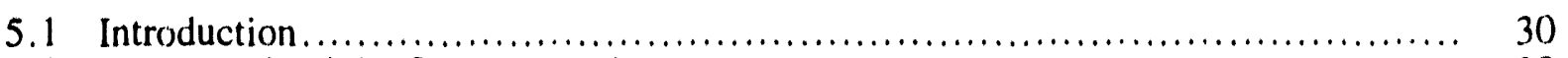

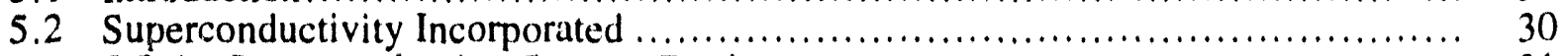

5.2 .1 Superconducting Storage Device .................................... 31

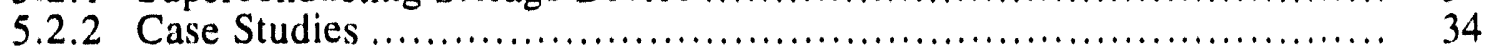

5.2.3 Market Evaluation for SSD ....................................... 36

5.3 Potential Application of HTS to Small-Scale SMES ......................... 38

6 Medium-Scaie SMES .................................................... 40

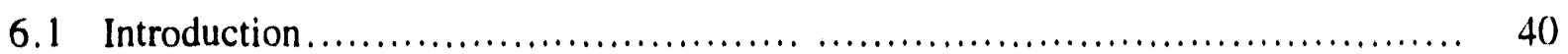

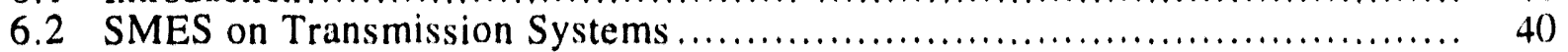

6.3 SMES for Customer Load Leveling ........................................ 41

6.4 SMES for Stationary Transportation Applications ........................... 42

6.5 Potential Impacts of HTS-Based SMES Systems ......................... 43

7 Flywheel Storage with Superconducting Magnetic Bearings ...................... 46

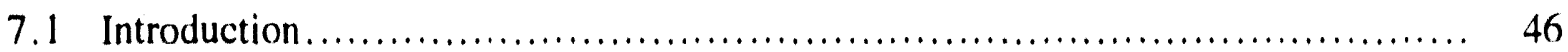

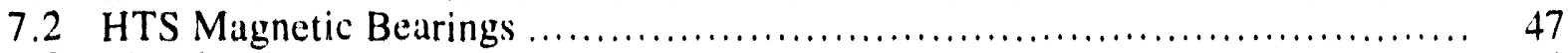

7.3 Flywheel Storage System................................................. 49

7.4 Argonne/Commonwealth Edison Flywheel Project................................. 52

7.5 European Community Flywheel Project ................................... 55 


\section{Contents (Cont.)}

8 Summary and Conclusions........................................................ 56

8.1 Superconducting Magnetic Energy Storage............................... 56

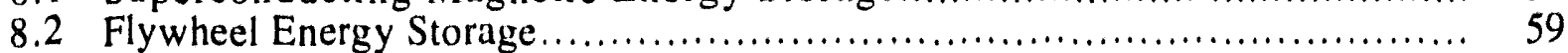

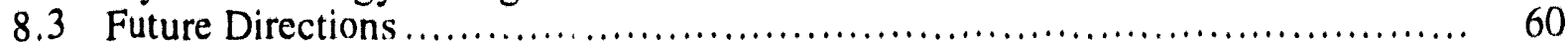

Appendix A: Power Conversion Systems ...................................... A-1

\section{Tables}

3-1 Scaling Dependence of SMES Components Upon Stored Energy ................ 11

4-1 Summary of SMES Units Built ............................................ 23

4-2 Ongoing SMES Design/Cost Studies and SMES Construction .................. 23

4-3 Comparison of Japanese and American Large-Scale SMES Designs .............. 25

4-4 Summary of Bechtel and Ebasco ETM Designs.............................. 27

6-1 Comparison of Radius, Surface Area, and Conductor Requirements

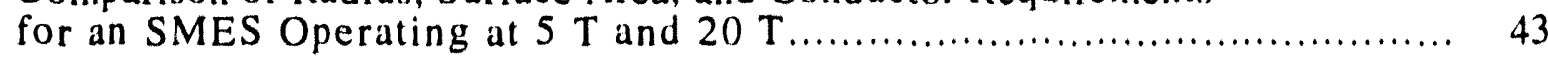

Figures

2-1 Block Diagram for Superconducting Energy Storage System ................... 6

2-2 Plot of SES Power versus Energy Requirements for Potential Applications....... 7

3-1 Cost Components for a Large-Scale SMES ................................ 12

3-2 Relative Superconductor Requirements Versus Aspect Ratio for Several Coil Geometries

3-3 Relative Cost of Cold Versus Warm Support Structure as a Function of SMES Stored Energy.

3-4 Components of Daily Refrigeration Load as a Funct ior of SMES

Stored Energy for SMES Coils Constructed in Competent Rock

4-1 Artist's View of Generic SMES Plant.

4-2 Relative Cost-Effectiveness of Large-Scale SMES for Diurnal Load Leveling as a Function of Daily Discharge Period 


\section{Figures (Cont.)}

4-3 SMES Coil Diameter for Construction in Soil and Rock versus

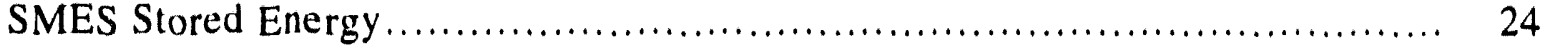

4-4 Artist's View of Trench Construction for In-Soil SMES Unit .................. 25

4-5 Cross Section for Bechtel $200 \mathrm{kA}$ Conductor for ETM...................... 28

4-6 Cross Section for Ebasco $50 \mathrm{kA}$ Conductor for ETM........................... 28

5-1 CBEMA Voltage Standard for Mainframe Computers ......................... 31

5-2 Cutaway Drawing of Magnet and Cryostat Assembly for Super conductivity Incorporates I MJ Superconducting Storage Device

5-3 Block Diagram of the Superconductivity Incorporated I MJ

Superconducting Storage Device...

5-4 Comparison of Specific Power and Energy Density for Several

Proposed Storage Systems.

5-5 Comparison of Costs for a Present and Future LTS-Based and HTS-Based SSD Unit

6-1 Illustration of Design for an HTS-Based SMES Module by W.J. Schafer Associates, Inc.

7-1 Picture of Composite Flywheel and Motor/Generator Unit as Assembled by Oak Ridge National Laboratory............................. 48

7-2 Illustration of Magnetic Bearing Built by Koyo Seiko Company in Japan......... 50

A-1 Schematic of a Six-Pulse, Three-Phase Graetz Bridge ......................... A-3

A-2 Schematic of Single-Phase Graetz Bridge ................................ A-3

A-3 Wave Forms Associated with a Single-Phase Graetz Bridge:

(a) for $\alpha=0^{\circ}$, (b) for $\alpha=45^{\circ}$, (c) for $\alpha=90^{\circ}$, (d) for $\alpha=135^{\circ}$, and (e) for $\alpha=180^{\circ}$.

A-4 Wave Forms (a) for a Six-Pulse Graetz Bridge, and (b) for a Twelve-Pulse Graetz Bridge. 


\title{
Superconducting Energy Storage
}

by

\author{
R.F. Giese
}

\section{Introduction}

Unlike other sources of energy, such as oil, natural gas, and gasoline, electricity is difficult to store. Once generated, electricity travels at nearly the speed of light over wires to the point where it is used. Consequently, as the demand for electricity varies, the amount of energy generated must be constantly adjusted to match the instantaneous demand.

Variations in demand occur over a period of time ranging from less than one second to one year. The daily or diurnal variations have received the most attention because of the magnitude and frequency (once per day) of variation. In North America, average daily power levels of 80-85\% of daily peak demand are common. However, daily minimum loads are typically only $50 \%$ of the daily maximum load. Since the most efficient and lowest cost units (base-load plants such as coal and nuclear) are designed to operate at or near full capacity, less efficient units designed for cycling must be used, thereby increasing the cost of generation.

Were a device available to store excess energy during the night for use during the following day, the total amount of installed capacity could be reduced, and the amount of the most efficient, base-load capacity could be increased. Superconductivity provides the potential for developing two types of efficient energy storage: (1) building large, high-field superconducting magnets to store energy in the form of persistent, circulating currents; and (2) flywheels, incorporating frictionless, superconducting magnetic bearings, to store energy in the form of kinetic energy. 1 In each case, electronic power conversion equipment is used to store and retrieve the electrical energy.

In principle, there are a wide variety of generation and energy storage technologies that could be used to satisfy diurnal variations in demand. Several technologies, such as cycling coal, gas turbines, and hydroelectric, are widely used today. Other technologies, such as pumped hydro, compressed air energy storage, and battery storage, are in limited use. Some proposed technologies, such as underground pumped hydro, superconducting magnetic energy storage (SMES), and flywheels employing magnetic bearings, show promise. Utilities will select the technology that provides the most performance at the lowest cost. Consequently, the cost and performance of superconducting energy storage (SES), such as SMES and flywheels with superconducting, magnetic bearings, is very important.

1 Although the superconducting bearing in a flywheel superconducting energy storage (SES) system is only a smull component in a flywheel system and does not actually store any energy, it may be required to achieve a high efficiency comparable to a SMES unit. 
There are several potential performance advantages of SES. First, the efficiency of SES could be greater than 9()$\%$. In contrast, the efficiency of other storage technologies ranges from 60 to $80 \%$. Secondly, SES operates at electronic speed and can switch from full charging to full discharging mode in une or two cycles (i.e., tens of milliseconds), except, possibly for battery storage, other storage technologies respond much slower (i.e., seconds to minutes). The ability to respond quickly increases the potential value of SES. In addition to diurnal storage, SES can provide spinning reserve, power conditioning, and system stabilization functions as well as compensation of load fluctuations at customer sites. Another advantage of SMES is the ability to perform frequent deep discharges without affecting the long lifetime of an SMES unit. Except for possible concerns relative to large de fringe fields associated with SMES units. SMES should be environmentally ben:gn. The rate at which energy is added to or withdrawn from a SMES unit depends upon the application. In the case of an SES unit used for power quality (Section 5) and power stability (Section 6), full charge and full discharge can occur within a second. For diurnal applications (Section 4), the relevant time-scale is hours, not seconds. The only limitations on how fast energy can be added to and withdrawn from an SES unit are due to (1) ac losses occurring within the coil and (2) the magnitude of voltage developed across the coil and consequently the power conditioning system (PCS). In each case, whether or not SES will be the technology of choice will depend upon its cost and level of performance.

The purpose of this work is to (1) examine possible applications of high-temperature superconductors (HTS) to the storage of electric power, in either SMES or in a flywheel employing magnetic bearings; (2) to establish performance goals for HTS; and (3) to assess the possible impact on design of going from conventional low-temperature superconductors (LTS) to ceramic HTS. A major aim is to identify issues that need further investigation. Detailed technical and economic assessments are beyond the scope of this work.

Section 2 presents a brief technical overview of SES. This overview is followed by a discussion of design trade-offs associated with SMES in Section 3. Sections 4, 5, and 6 summarize large-, small-, and medium-scale SMES applications and devices. The use of HTS magnetic bearings in flywheels is presented in Section 7. Section 8 presents a summary and a set of conclusions. 


\section{Superconducting Energy Storage}

\subsection{Introduction}

In this report, we consider two forms of energy storage based upon superconductivity: (1) SMES, and (2) flywheels incorporating magnetic bearings. Compared to conventional storage options such as pumped hydro, batteries, and compressed air energy storage, the SES systems offer the potential for very high, round-trip efficiency which could improve the economic attractiveness and alter how SES devices are used in an electric utility system.

\subsection{Superconducting Magnetic Energy Storage}

The flow of surrent through a wire creates a magnetic field which can provide a means of storing electrical energy. In principle, any large magnet can be used to store electrical energy. The volumetric energy density, $E$ in $\mathrm{J} / \mathrm{m}^{3}$, associated with a magnetic fielc is given by:

$$
E=\int \boldsymbol{B} \cdot d \boldsymbol{H}=\frac{\boldsymbol{B}^{2}}{2 \mu \mu_{0}}
$$

where:

$B$ is the magnetic field intensity in $\mathrm{T}$,

$\mu \quad$ is the average relative magnetic permeability, and

$\mu_{0} \quad$ is the magnetic permeability of free space.

To achieve a large volumetric energy density, both a small $\mu$ and a large $\mathrm{B}$ are necessary. Using conventional wires, the usual way to achieve a high magnetic field is to use a magnetic material such as iron. ${ }^{2}$ An electromagnet having an iron core can achieve magnetic fields as high as $2 \mathrm{~T}$. However, the $\mu$ for iron is typically about 1,000. Using these values results in an $E$ of only $0.44 \mathrm{Wh} / \mathrm{m}^{3}\left(0.44 \times 10^{-3} \mathrm{Wh} / \mathrm{l}\right)$. In comparison, a conventional, lead-acid car battery has an energy density of approximately $80 \mathrm{Wh} / \mathrm{l}, 200,000$ times greater.

The use of superconducting wire can increase $E$ dramatically by increasing the maximum attainable magnetic field and eliminating the need for a magnetic material. Assuming a $B$ of $6 \mathrm{~T}$ and a $\mu$ of 1 (corresponding to an air-core magnet), yields an energy density of $4 \mathrm{Wh} / \mathrm{l}$. This is

2 Fields as high as $25 \mathrm{~T}$ have been achieved using conventional copper conductors without an iron core. However, such Bitter magnets have large losses due to Joule heating. 
still a factor of 20 less than a conventional, lead-acid battery. However, since the magnetic field can extend well beyond the structure used to generate the magnetic field, and since the total stored energy, $\mathbf{E}$, is obtained by integrating $E$ over all space, $E$ can be substantial. Alternately, $\mathbf{E}$ is given by:

$$
E=\frac{L I^{2}}{2}
$$

where:

$\mathrm{L}$ is the inductance of the coil in henries, and

I is the total current flowing through the coil in amps.

Since there are no electrical losses associated with a circulating constant current in a superconducting magnet, such a device should be $100 \%$ efficient. In practice, there are refrigeration losses associated with keeping the device cold. In addition, the electricity in a grid is alternating current. Thus, the current must be converted from ac to dc to charge the magnet, and from dc to ac to discharge the magnet. The electronic device required to perform these conversions entails some loss, although this is usually quite small. Thus, an SMES device has the potential for storing and retrieving energy quite efficiently.

With the construction of large, superconducting bubble chambers, the ability of a large magnet to store electrical energy was realized by researchers in Europe and the United States during the early 1960s. In 1963, J. Stekly proposed a design for a small SMES for use in aircraft, etc. In 1970, M. Ferrier [1] proposed using a single, large, centrally located SMES unit that would satisfy all of the cyclic power requirements of Electricite de France. He proposed using a toroidal coil. Due in part to the large structure required to contain the large Lorentz forces generated by such a magnet, the initial estimates of capital and development cost for such a magnet were high, and no work was done beyond this simple conceptualization.

The present-day SMES program originated at the University of Wisconsin in 1971 when Professors Boom and Peterson began studying the interaction of an energy storage unit connected to an electric grid through a mutiphase Graetz bridge [2,3]. In 1972, the U.S. Atomic Energy Commission provided funding to Los Alamos National Laboratory to perform a feasibility study and outline a development program. Los Alamos performed an economic analysis of SMES and concluded that a stainless steel structure required to contain the Lorentz forces would be prohibitively expensive [4]. Hassenzahl et al. proposed replacing the traditional, external, stianless steel support structure which was housed within the cryostat and was referred to as a "cold structure" with a warm containment structure [5]. It was found that by building the magnet in situ in rock, significant cost savings could be realized. Warm structure is the preferred method for containing Lorentz forces in all proposed, large-scale SMES designs today. 


\subsection{Flywheel Storage}

Instead of storing energy in the magnetic field associated with a circulating current, energy can be stored mechanically in a rotating flywheel. In this case, $\mathbf{E}$ is given by:

$$
E=\frac{J w^{2}}{2}
$$

where:

$\mathrm{J} \quad$ is the flywheel moment of rotational inertia, and

$\omega \quad$ is the flywheel angular velocity.

To achieve a high stored energy, both a large $J$ and a large $\omega$ are desirable. However, the large centripetal forces present in flywheels provide practical limits to both $J$ and $\omega$. Proposed flywheel designs have a volumetric energy density comparable to or better than conventional, lead-acid batteries. Losses associated with flywheels come from three sources: (1) windage losses due to friction with air, (2) bearing losses due to friction, and (3) conversion losses associated with the motor/generator used to put electrical energy into and to obtain electrical energy from the flywheel. The windage losses are reduced by operating the flywheel in a vacuum. The conversion losses are comparable in magnitude to those of an SMES and are primarily due to the electronic ac-dc-ac conversions required to accept a range of $\omega$ values during charging and discharging. Bearing losses can be reduced through the use of magnetic bearings. However, conventional magnetic bearings are unstable and require the use of an electronic, feedback circuit to ensure mechanical stability. HTS can be used to provide mechanical stability without incurring the energy penalty associated with the electronic feedback circuits. However, some energy must be expended for the refrigeration that maintains the HTS in the superconducting state.

\subsection{SES System}

Figure 2-1 presents a block diagram of a typical SES system. A transformer provides the connection to the electric utility system and reduces the operating voltage to several $\mathrm{kV}$; a power conditioning system (PCS), which is a high-power, solid-state device provides an ac-dc interface between the grid and the SES; a refrigeration system that keeps the SES cold; and a controller coordinates the operation of all components. An external demand signal provides input to the controller.

An SMES is essentially a de device. Consequently, a power-conditioning system is needed to convert ac power to dc power during SMES charging, and from dc power to ac power during SMES discharging. The controller controls the operation of the PCS to provide the desired amount of power during charge and discharge. 


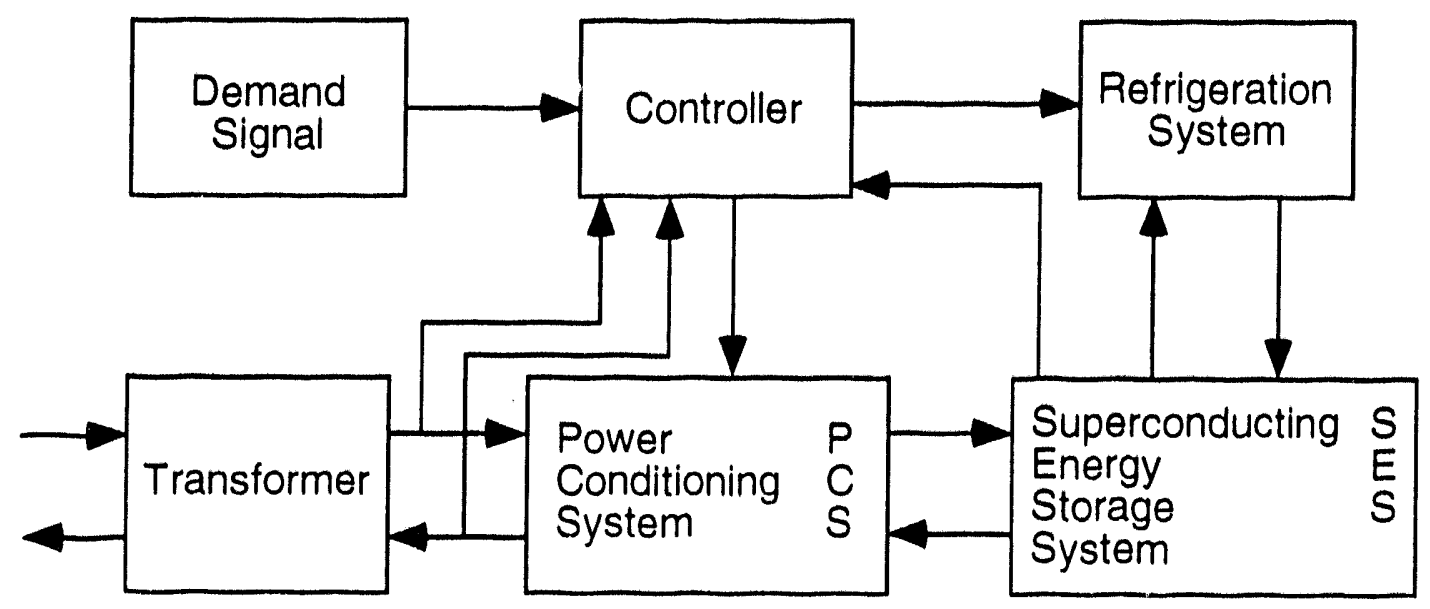

FIGURE 2-1 Block Diagram for Superconducting Energy Storage System

Energy is added (removed) from a flywheel through a motor/generator set which is physically attached to the shaft of the flywheel. Although the flywheel is intrinsically an ac device, the frequency of the flywheel varies during charge and discharge. The PCS acts as an ac dc/dc ac frequency converter to match the flywheel frequency to the grid frequency (either 50 or $60 \mathrm{~Hz}$ ). The converter controls operation of the system to provide the desired amount of power.

Appendix A describes how a PCS operates, some of the tradeoffs involved in design of a PCS, and how PCS technology has evolved over time.

\subsection{Possible SES Applications}

The initial perceived application of SES was to provide diurnal storage for load-leveling on the electric utility grid. The SES would be charged at night using excess, low-cost, base-load power and discharged during the following day to displace the use of high-cost, peaking power. The resulting savings would come from two sources: (1) the substitution of low-cost, base-load fuel for high-cost, peaking fuel, and (2) elimination of some peaking generators (or delaying the purchase of additional generation).

In the first case, savings would accrue when the cost of the base-load fuel required to generate $1 \mathrm{MW}$ divided by SES efficiency is less than the cost of the peaking fuel required to generate $1 \mathrm{MW}$. In many cases, additional savings accrue because the base-load units can operate continuously during periods of low load. Most base-load power plants run best at full power. Running at lower power levels results in additional operating costs. First, unit efficiency decreases at part load, which requires the consumption of additional fuel. Second, maintenance costs increase as a unit is forced to cycle up and down. By charging an SMES unit at night, the baseload unit can operate efficiently at constant power. In many cases, these cost savings are greater than the fuel-substitution benefits. 
In the second case, savings would accrue as long as the SES was not fully discharged during the periods of peak demand, which only occur a few times a year. The capital cost and efficiency of the SES unit are of primary concern for this application. Such an SES device is characterized by both a large power and a large energy rating. Large-scale SMES will be discussed in Section 4.

Subsequently, a number of additional applications have been proposed. The units for these applications have a range of power and energy ratings and are summarized in Figure 2-2. The $x$ axis displays the required energy capacity, while the $y$ axis displays the power rating of the SES. Large-scale, diurnal, load-leveling SES is found at the upper right-hand corner, requiring a storage capacity of 100-10,000 MWh and a power rating of 100-3,000 MW. In general, an SES for diumal load leveling is capable of full charge and discharge for several hours.

In addition to providing load-leveling for generation capacity, large-scale SES can be used to level the load on existing transmission lines, thereby providing for additional transmission capacity during periods of peak transmission load. SES can also be used to provide dynamic

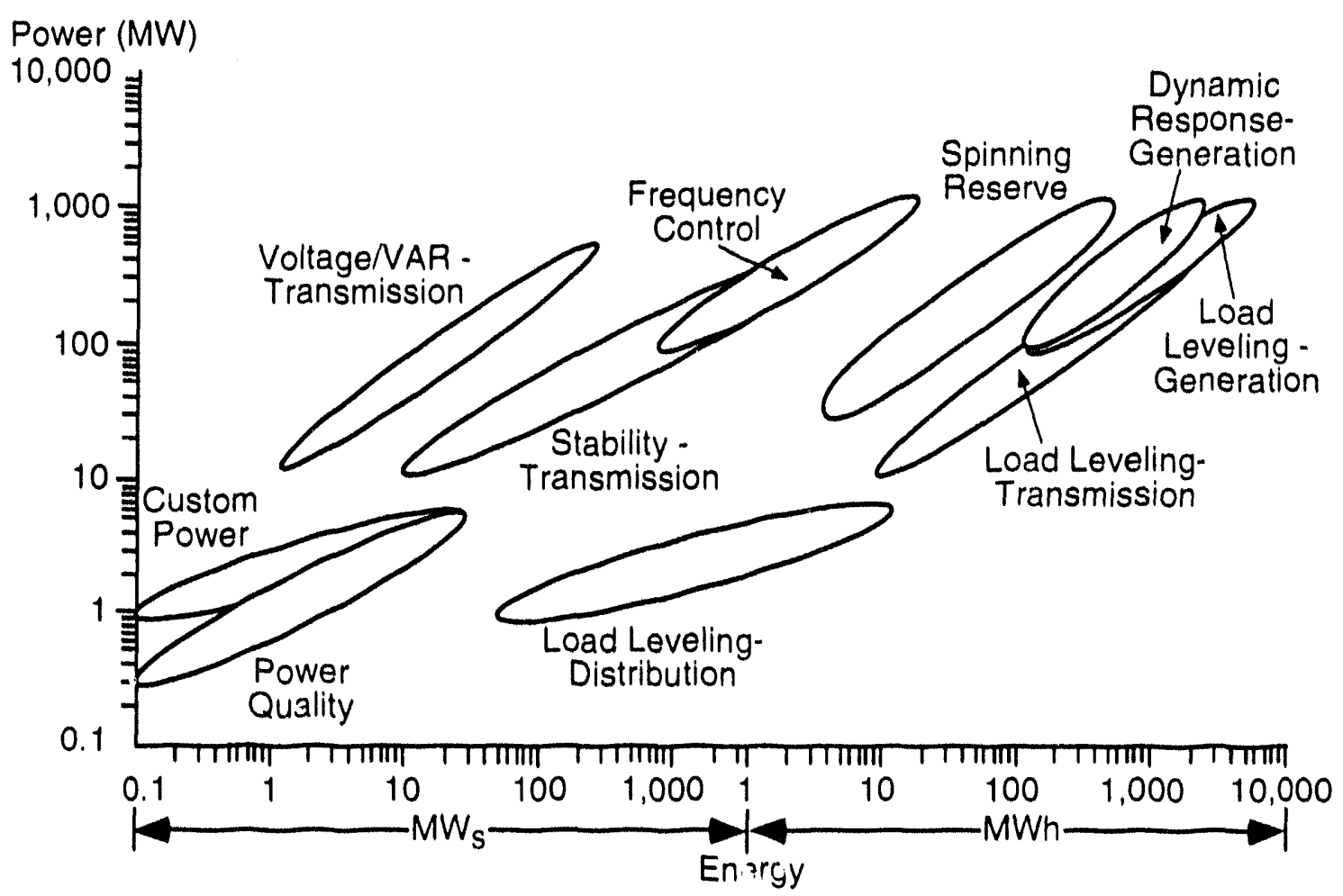

FIGURE 2-2 Plot of SES Power versus Energy Requirements for Potential Applications 
response during periods of rapidly changing load. SES can provide the spinning reserve needed should a large, base-load unit trip. ${ }^{3}$ Although these four applications have similar SES power and energy requirements, it would be difficult for a single SES unit to satisfy all of these applications simultaneously. ${ }^{4}$

At the other end of the spectrum are applications for small-scale SES for custom power and power quality. In these applications, the SES is used to provide short bursts of power, such as during motor start up, to prevent voltage sags and to continue operation of critical loads during momentary power outages. A large amount of power is required for a fairly short period ranging from several cycles up to several seconds. The resulting SES energy capacity and power rating ranges from 0.1 to $30 \mathrm{MWs}$ and 0.3 to $3 \mathrm{MW}$, respectively. Basically, these devices are a power conditioning system with a very small energy storage capacity. The primary requirement for smallscale SES is the ability to respond rapidly to changing load conditions. Cost, mass, and volume are of secondary consideration. With the growth of electronic controls and devices and their sensitivity to voltage fluctuations, the market for custom power and power quality is growing. ${ }^{5}$ Small-scale SMES will be discussed in further detail in Section 5.

A number of medium-scale SES applications fall between these two extremes. These include load leveling at the distribution substations or on the premises of large industrial/commercial customers and enhancements in the ability of the transmission system to carry large amounts of power. These applications require an SES discharge capability ranging from $0.1 \mathrm{~s}$ up to an upper limit of $1 \mathrm{~h}$ SES energy capacity ranges from approximately $1 \mathrm{MWs}$ up to $10 \mathrm{MWh}$ while power levels range from I $\mathrm{MW}$ to 1,0(0) MW. Medium-scale applications of SMES will be discussed in further detail in Section 6.

${ }^{3}$ To prevent the system from becoming unstable should a large generator trip, utilities are forced to maintain sufficient capacity in a standby state to make up for the lost generation. Typically, these units are gas turhines with relatively high fuel costs. SES provides the opportunity to reduce spinning reserve requirements, thereby reducing hot standby of reserve units resulti, ? 1.1 reduced fuel consumption and associated emissions.

4 Either additional power and energy capacity must be provided, the SMES unit must be derated with regard to load leveling, or the SMES can only be used to provide additional uses during certain portions of the charge-discharge cycle. In addition. SES siting requirements may be different for each application.

5 Power quality usually refers to the quality of power being delivered by the utilities and includes facturs such ats harmonics, voltage sags, and voltage spikes. 


\section{Design Tradeoffs for SMES}

\subsection{Introduction}

SMES was initially conceived as a means of storing energy to provide diurnal load leveling. The potential advantages of SMES included the ability to respond to load changes very rapidly (at electronic speeds, within one or two cycles) and with very high efficiency. However, because there were existing alternatives for storing energy (such as pumped hydro and customerside-of-the-meter storage, as well as emerging storage technologies, such as compressed air energy storage [CAES| and batteries), SMES would have to compete on the basis of cost with these alternatives.

A simple, geometric argument indicates that the cost of SMES, in $\$ / \mathrm{kWh}$, should decrease with increasing SMES size. As shown in Section 2, the total energy stored in an SMES should be proportional to the volume of the SMES and, consequently, should scale as the radius cubed, R. ${ }^{3}$. However, the cost of an SMES is mainly related to the amount of superconductor and cryostat material, which should be proportional to the surface area and, thus, scale as $\mathrm{R}^{2}$. Consequently, the cost of SMES, in $\$ / \mathrm{kWh}$, should scale as $1 / \mathrm{R}$ or $1 / \mathrm{E}^{1 / 3}$. Thus, a 5 MWh SMES would have a unit cost that is 10 times as high as a 5,000 MWh unit. Consequently, large-scale SMES units have received the most attention by researchers.

Recently, there has been renewed interest in small-scale SMES units. This has come about for two reasons: (1) the discovery of HTS, and (2) the growing concern associated with power quality issues. The primary advantage of HTS compared with LTS is their reduced refrigeration requirements. Since the refrigeration load is expected to be proportional to the surface area of the coil, refrigeration is expected to represent a larger fraction of total.ivES cost as the size of the SMES unit decreases. Consequently, HTS are likely to have the largest impact on smaller-sciale SMES units. As pointed out in Section 2, power quality only requires a very small SMES unit on the order of a MWs $(-300 \mathrm{Wh})$ of capacity. In addition, the conductor is a relatively small part of the SMES cost; consequently, the present high cost of BSCCO powder-in-silver tube wire will not be detrimental in a small-scale SMES.

Much effort has gone into optimizing the design of a large-scale SMES. Various magnet designs have been considered. Methods for containing the large hoop stress imposed by the Lorentz forces acting upon the magnet have been analyzed. The effect of different aspect ratios. operating magnetic field strength, operating voltage, and current values on SMES cost have been analyzed. The remainder of this section describes how the various components of an SMES unit are expected to scale with size and how alternative magnet designs affect cost. 


\subsection{Scaling Laws for SMES}

To understand how some of the components of SMES affect cost, it is useful to determine how various magnet parameters scale with the magnetic field intensity $(\mathbf{B})$ and stored energy $(\mathbf{E})$. A number of groups have developed scaling relations. The one presented below was developed by the SMES group at the University of Wisconsin [6]. Equation 3-1 below expresses the relationship between SMES radius $(R)$, conductor requirements (IS), and magnet surface area (A) as a function of $\mathbf{B}$ and $\mathbf{E}$.

$$
\begin{gathered}
R[m]=G E^{1 / 3} B^{-2 / 3} \\
I S[A m]=Q_{i s} E^{2 / 3} B^{-1 / 3} \\
A\left[m^{2}\right]=Q_{a} E^{2 / 3} B^{-4 / 3}
\end{gathered}
$$

where $G, Q_{i s}$, and $Q_{a}$, are quality shape factors that depend upon the type of coil and the coil aspect ratio. For a fixed type of coil, aspect ratio, and $\mathbf{E}$, higher $\mathbf{B}$ results in a smaller coil radius $\mathbf{R}$, less IS, ${ }^{6}$ and smaller magnet surface area $A$, all of which may lead to lower SMES cost.

An important consideration in the design of an SMES is handling the large forces that the magnetic field exerts on the conductor. These Lorentz forces are proportional to the cross product of the magnetic field at the conductor and the current flowing through the conductor. In any SMES design, there is always an outward furce due to the magnetic field inside the conductor and an inward force due to the magnetic field outside the conductor. For a large-aspect-ratio solenoid, as typically discussed in physics texts, the inside field is large and the outside field is small, resulting in a net outward force proportional to $\mathbf{B}^{2}$. 7 This force induces a tensile hoop stress within the conductor. In a typical magnet, stainless steel straps are used to contain this outward force. The cross section of the straps is chosen so that the maximum stress in the steel is well below the elastic limit. It turns out that the mount of structural mass required to contain the Lorentz force is related to the total energy stored. The virial theorem defines the amount of structural mass $\left(M_{s t}\right)$ required to store energy $(\mathbf{E})$ as follows:

$$
M_{s t}=\left(1+2 Q_{c}\right) \rho_{s t} E / \sigma_{s t}
$$

where $Q_{c}$ is a compressive quality factor between 0 and $1, \sigma_{s t}$ is the structure density, and $\sigma_{s t}$ is the average design tensile stress. It was sho' ${ }^{\prime \prime l ~ e a r l y ~ o n ~ t h a t ~ a ~ m a n-m a d e ~ s t r u c t u r e ~ w o u l d ~ b e ~ t o o ~}$

6 Although IS (Am) of conductor is required, the $J_{c}$ of the superconductors will decrease with increasing $B$, which could result in the need for more supercondutor $(\mathrm{kg})$, and the cost of SMES could actually go up.

7 One way to reduce the Lorentz force is to increase the magnitude of the magnetic field outside the magent (fringe field). This results in a large compensating inward force and a lower net force on the conductor. 
expensive to make a large-scale SMES economically competitive. To reduce SMES cost, the present large-scale SMES designs rely on the use of "free" earth or rock. These materials however, have a significantly lower maximum working tensile stress than man-made materials. Consequently, the maximum net working radial pressure for these materials determines the maximum magnetic field allowed at a given radius.

For a fixed type of coil, aspect ratio, and $\mathbf{B}$, the coil radius, amount of conductor, and magnet surface area increase more slowly than $\mathbf{E}$. Thus, total SMES cost per unit of stored energy decreases with increasing $\mathbf{E}$. A breakdown of SMES cost components indicates that the total cost of an SMES plant per energy stored CE is given by Equation 3-3.

$$
C E=a / E^{1 / 3}+b+c / E
$$

where $a, b$, and $c$ are constants. Table $3-1$ shows how individual cost components scale with stored energy $\mathbf{E}$.

The cost of most SMES components increases more slowly than E, favoring large-scale SMES units.

TABLE 3-1 Scaling Dependence of SMES Components Upon Stored Energy

\begin{tabular}{lll}
\hline \multicolumn{1}{c}{ Component } & Parameter & $\begin{array}{c}\text { Energy } \\
\text { Dependence }\end{array}$ \\
\hline Conductor requirements (Am) & IS & $E^{2 / 3}$ \\
Hellum system: dewar, dump system, shields, and vacuum & $A$ & $E^{2 / 3}$ \\
External structure: trench, struts & A & $E^{2 / 3}$ \\
Reirigeration & A, I, constant & $E^{2 / 3}, E^{1 / 2}, E^{0}$ \\
Internal axial structure and structure for energy dissipation & E, A & E, $E^{2 / 3}$ \\
Power conditioning & E, constant & E, $E^{0}$
\end{tabular}




\subsection{Cost Components}

Figure 3-1 displays the relative cost components for various sized SMES units based upon the cost developed for the large-scale (5,00) MWh) SMES design proposed and costed by Bechtel under contract to Los Alamos National Laboratory 171. For purposes of this plot, the basc cost has been scaled as a function of the stored energy $(\mathbf{E})$ based on scaling laws developed by the University of Wisconsin |81. For small SMES sizes, refrigeration costs become very important. HTS has the potential to reduce refrigeration costs significantly for small SMES sizes. At large sizes, PCS costs become important. This is because the PCS consists of modules. Going to a larger SMES size increases the number of modules required, but does not reduce the price of a module. Two of the largest cost components are the conductor cost and the construction cost associated with the trench used to house the coil. These two components are largely independent of $\mathbf{E}$.

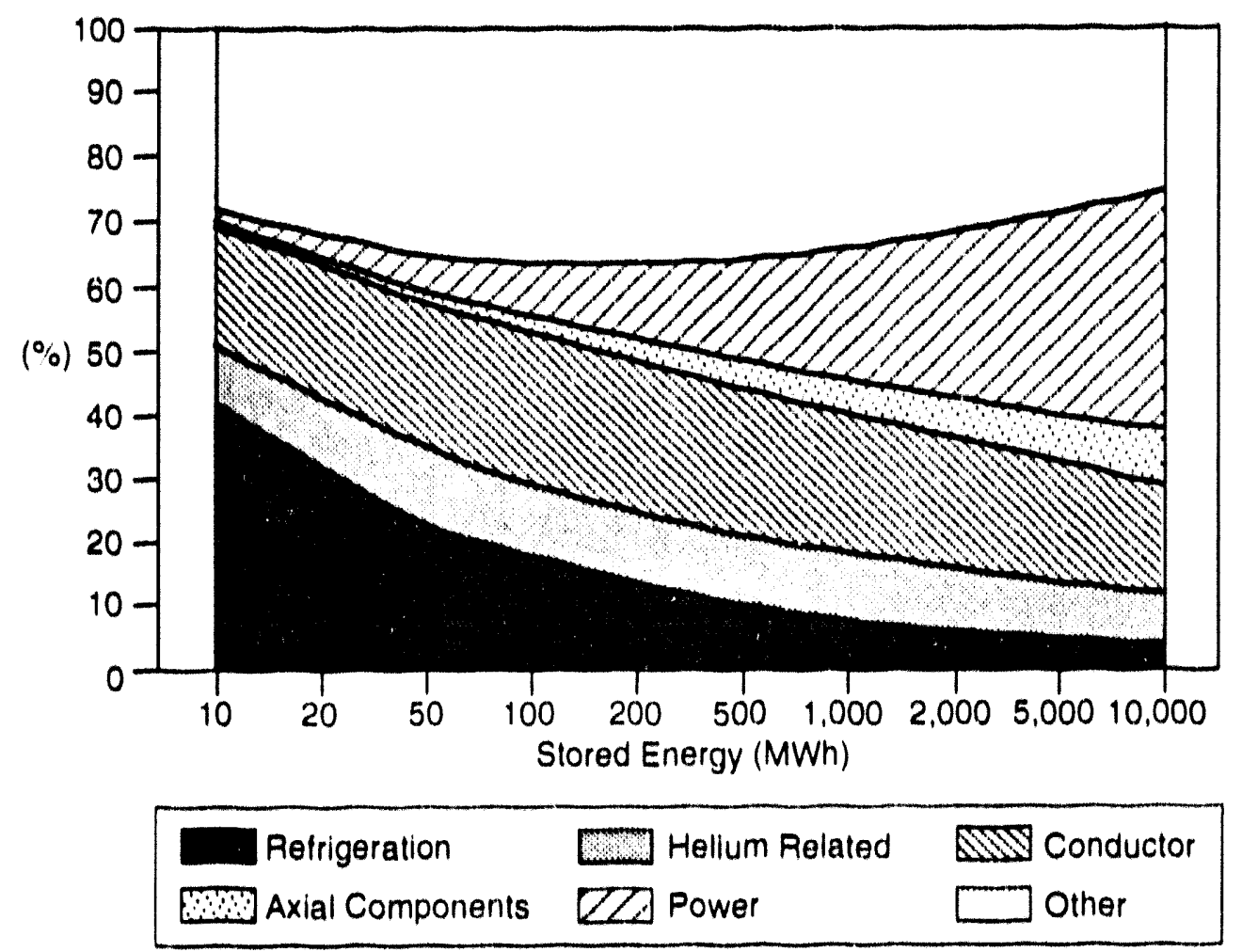

FIGURE 3-1 Cost Components for a Large-Scale SMES 


\subsubsection{Conductor}

The conductor accounts for approximately $20 \%$ of the SMES capital cost and depends primarily on the coil aspect ratio $\beta$ and the critical current density $J_{c}$ under operating conditions. Traditionally, the conductor is priced per unit of conductor assembly mass $C_{\$}(\$ / k g)$. Consequently, the cost of the conductor assembly $\mathrm{C}_{\$}$ is given by Equation 3.4 as:

$$
C_{S}=C M_{S} M_{c}=C M_{S} \rho_{c} I S / J_{C}
$$

where:

M. is the mass of the conductor assembly $(\mathrm{kg})$,

$\rho_{c} \quad$ is the density of the conductor assembly $\left(\mathrm{kg} / \mathrm{m}^{3}\right)$,

IS is the conductor requirement $(\mathrm{A} \mathrm{m})$, and

$\mathrm{J}_{\mathrm{c}} \quad$ is the critical current density of the conductor assembly $\left(\mathrm{A} / \mathrm{m}^{2}\right)$.

Thus, a small IS and a large $J_{c}$ minimize the cost of the conductor assembly. To increase $J_{\text {. }}$ several SMES designs call for operating the conductor at $1.8 \mathrm{~K}$ and lower magnetic field, rather than the traditional $4.2 \mathrm{~K}$.

The only other variable available to the SMES designer is IS. As shown in Equation 3.1. for a fixed B and E. IS is proportional to $Q_{i s}$. As part of the Los Alamos SMES design effort $|9|$. W.V. Hassenzuhl determined the dependence of $Q_{\text {is }}$ on $\beta$ for several types of coil. Figure 3-2 illustrates $Q_{\text {is }}$ for three proposed SMES coil geometries: solenoidal, toroidal, and poloidal. The best geometry is a thin-walled solenoid with a $B$ of about 0.2 with a corresponding $Q_{i s}$ of $5(6)$. For lower values of $\beta$ corresponding to the low-aspect-ratio (LAR) region, coil effectiveness decreases slowly (e.g. $\left.Q_{1 s}(.0 .02)=700\right)$. However, some designs choose to operate in this region to reduce the magnitude of outward pressure (see Section 4). The best toroidal coil requires more than twice as much conductor as the best thin-walled solenoidal coil, and the minimum occurs for much higher $\beta$ value of approximately 0.6. However, since the fringe field associated with a toroidal coil is much smaller than that of a thin-walled solenoid, a toroidal design may be required for applications where minimizing fringe fields is important. An optimum toroidal coil also tends be more rompact and incurs higher hoop stresses, requiring more support mass, than for a thinwalled solenoid. Thus, unless fringe field is a major concem, a thin-walled or L.AR solenoid is best. 


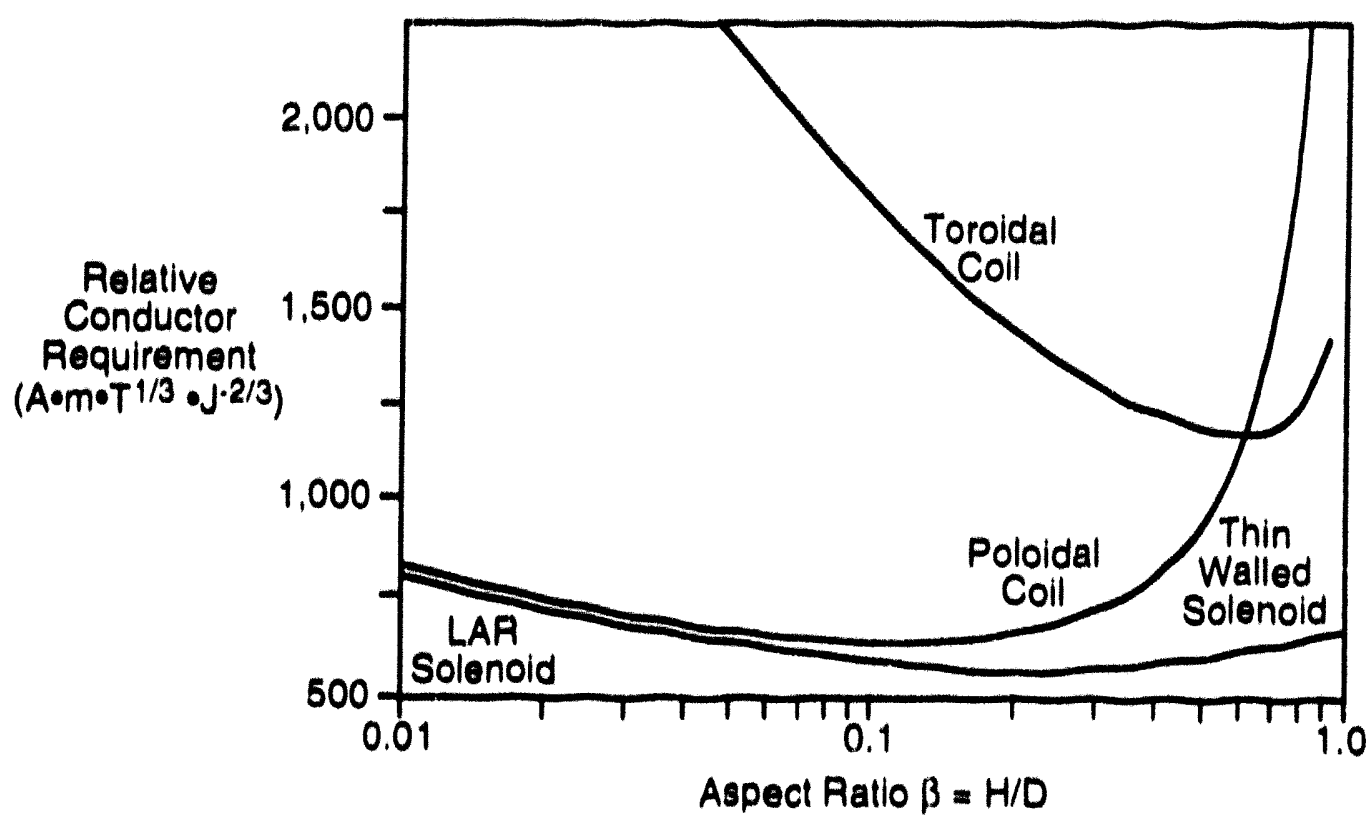

FIGURE 3.2 Relitive Superconductor Requirements Versus Aspect Ratio for Several Coll Geometries

\subsubsection{Struoture}

Large forces build up during the operation of an SMES plant. These forces take two forms. First, during cool down from room temperature to the cryogeni : operuting temperuture, the cable and associuted structures are subjected to a thermal contraction on the order of $0.5 \%$. This exerts a large inward force. Second, as the mugnet is energized, an outward force proportional to $\mathrm{B}^{2}$ develops on the conductor. Under normal operating conditions, this loud cycles once a duy from a low value when the SMES unit is discharged, 10 a maximum when the SMES unit is fully charged. In principle, a single structure could be developed to contain both forces. In practice. different structures, especially in the case of large-scule SMES units, have developed.

Two approaches have been developed to handle stresses developed during cool down. In the first approach, the cable assembly contuins a set of ripples. As the cable assembly cools, the ripples absorb the contraction, and become less rippled. In the second approach, a rigid cable assembly is pre-stressed radially outward (i.e., compressed) at room temperature. As the cable assembly cools, stress is reduced, passes through zero, and finally reverses, reaching a tensile stress at the operating temperature comparable in magnitude to the compressive stress at room temperature. A set of stainless steel cable inserts is used to handle the resulting stress thereby maintaining a relatively low level of stress within the conductor. Calculations indicate that both techniques will work.

The main cost in the "other" category in Figure 3-1 is associated with the structure required to contain the force that develops during the cyclic charging and discharging of the SMES coil. 
Two approaches have been developed to handle these forces. The first approach employs a set of stainless steel bands which surround the coil. Typically, these bands are pre-stressed radially inward when the coil is deenergized. As the coil is energized, the stress is reduced, passes through zero, and increases to a maximum stress at full energization. This technique is commonly referred to as using "cold structure" because the containment structure is located within the dewar and operates at cryogenic temperatures. Cold structure has been shown to be too expensive 10 use in large-scule SMES units.

In large-scale SMES designs, a set of struts has been proposed to transfer the forces to an external structure, typically soil or competent rock. The struts are made from either fiberglass and epoxy, or fiberglass-reinforeed plastic. Since this structure exists outside the dewar, it is commonly referred to as "warm structure." The primary advantage of warm structure is that the rock or soil used to contain the forces is free. However, this scheme requires either digging a trench in the case of soil, or boring a tunnel in the case of rock. Additionally, the maximum working stress for either rock or soil is two to three orders of magnitude less than for stuinless steel. To achieve lower working stress, large-scale SMES designs use LAR coils. Although this increases the amount of conductor mass, it reduces the maximum net magnetic field at the surfice of the coil and thereby reduces the force. A disadvantage of this approach is that fringe fields are increused.

As the size of the SMES unit is decreased, the amount of structural mass also decreases. At some point, it becomes more economical to use cold structure than warm structure, ${ }^{K}$ Calculation of the crossover capacity depends upon the assumptions made for each design. Figure 3-3, taken from reference 10, shows that the crossover for one set of assumptions for a toroidal coil occurs at an SMES capacity of approximately 10,00) MJ (3 MWh). The crossover occurs at a somewhat higher value for a solenoidal coil design.

\subsubsection{Power Conditioning System}

The PCS is an integral part of an SMES unit. As pointed out earlier, for a fixed charge/discharge cycle, the relative cost of the PCS becomes more important as the SMES capacity grows. For a 10,000 MWh unit, the PCS accounts for approximately one third of the total cost. For small-scale SMES units with short charge/discharge cycles the cost of the PCS is even more importunt and represents over half of the total cost.

The choice of the maximum current $I_{\max }$ through the coil and the maximum voltage $V_{\max }$ across the coil determine the cost of the PCS. Typically, a single PCS module can withstand a $V_{\text {max }}$ of 5 to $6 \mathrm{kV}$. By putting two modules in series, a $V_{\max }$ of $101012 \mathrm{kV}$ is ohtainable. Putting more than two units in series tends to reduced the reliability of the PCS, increases the PCS

8 In the case of a toroidal coil, geometsy may make it difficuit to design a suitable warm structure 


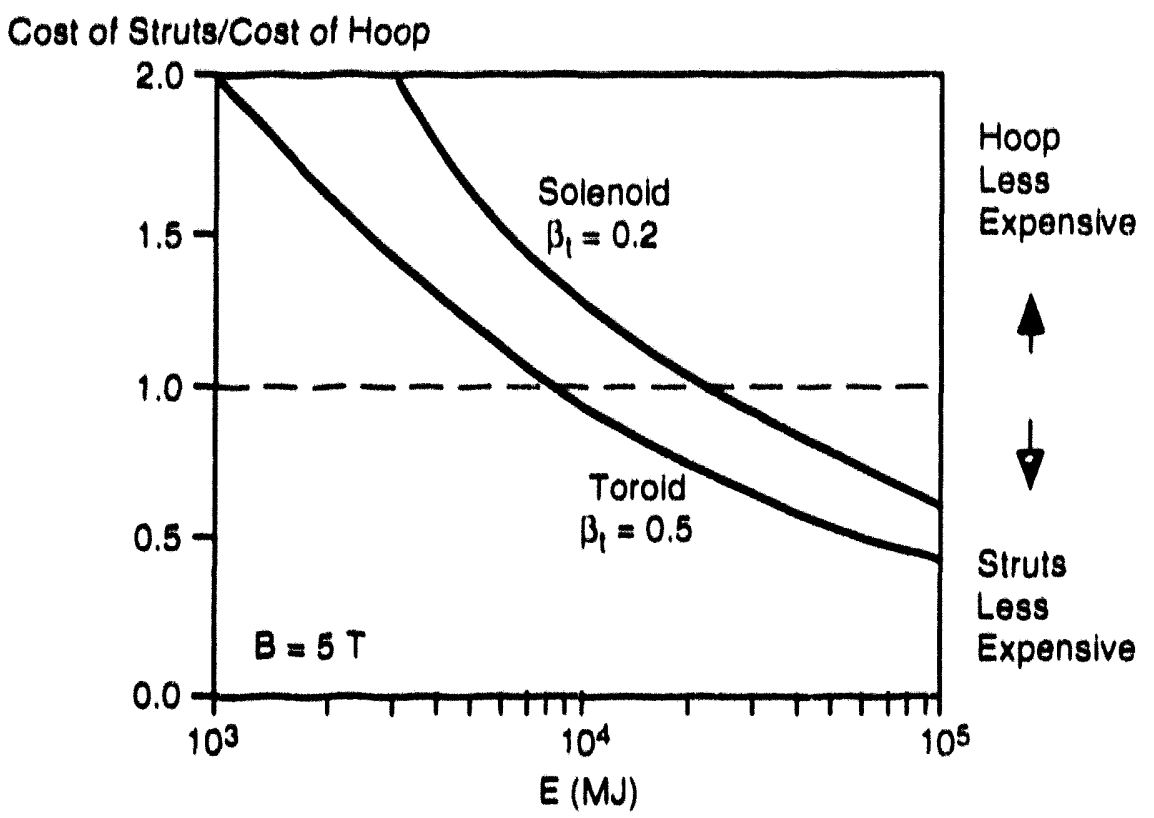

FIQURE 3-3 Relative Cost of Cold Versus Warm Support Structure as a Function of SMES Stored Energy

cost, and increases the risk of turn-to-turn voltage breakdown within the coil. Consequently, most large-scale SMES designs use a $V_{\max }$ of $10-12 \mathrm{kV}$. As the SMES unit is discharged, the current I flowing through the coil decreases. Since the power being delivered by the SMES unit is given by the product of $I$ and $V$, a constant power discharge requires that $V$ increase as I decreases. Consequently, assuming a constant power discharge, the value of $V_{\text {max }}$ determines the amount of energy left in the SMES unit at full discharge." For a fixed discharge power $P$ :

$$
\begin{aligned}
I_{\min } & =P / V_{\max } \\
E_{\min } & =L \frac{I_{\min }^{2}}{2} \\
& =\frac{P^{2}}{V_{\max }^{2} I_{\max }^{2}} E_{\max }
\end{aligned}
$$

9 More energy could be withdrawn from the SMES by maintaining a fixed $V_{\text {max }}$ and decreasing the level of power during discharge. However, this would impose constraints on the SMES operation during discharge and reducing its potential value. 
where:

$E_{\max } \quad$ is the maximum energy stored in the coil,

$E_{\min } \quad$ is the amount of energy remaining in the coil at full discharge,

$L \quad$ is the inductance of the coil, and

$I_{\min } \quad$ is the amount of current flowing in the coil at full discharge.

Selection of the correct $I_{\max }$ involves a tradeoff. For a fixed $E_{\max }$, the larger the value of $I_{\max }$, the larger the fraction of energy that can be extracted from the coil and the fewer number of turns of conductor that is required. However, a larger the value of $I_{\text {max }}$ requires larger current leads and higher associated losses, as well as a higher PCS cost and more current flows through the conductor.

\subsubsection{Refrigeration}

The refrigeration load consists of three components: internal $l^{2} R$ losses within the current lead $(E)$, heat leak along the current lead $\left(E^{1 / 2}\right)$, and conduction/radiation heat leaks through the dewar wall $\left(E^{2 / 3}\right),{ }^{10}$ Figure 3.4 illustrates the components of daily refrigeration load as a percentage of the stored energy $E_{\max }$ as a function of $\mathbf{E}$ for an SMES coil installed in competent rock. For an SMES capacity in excess of 5,000 MWh, the total refrigeration load represents only $2 \%$ of the stored energy. However, for a $100 \mathrm{MWh}$ unit. $13 \%$ of the stored energy is consumed in refrigeration. This greatly reduces the effective efticiency of the SMES unit.

\subsection{Potential Role for HTS}

The use of HTS could greatly reduce both the capital and operating costs associated with refrigeration of an SMES unit. As shown in Figures 3-1 and 3-4, the relative importance of refrigeration grows as the SMES capacity decreases. In principle, the use of an HTS conductor in an SMES unit might greatly reduce or even eliminate the helium-related and refrigeration costs. This could reduce the capital cost of a 5,000 MWh SMES unit by up to $10 \%$ and the capital cost of

10 In principle, there is also an ac loss within the superconductor during charging and discharging of the SME:S unit. However, the relatively long charge and discharge periods (many hours) associated with load leveling make this loss quite small. However, ac losses can be quite large in some small-scale SMES applicattions isee Section 5). 


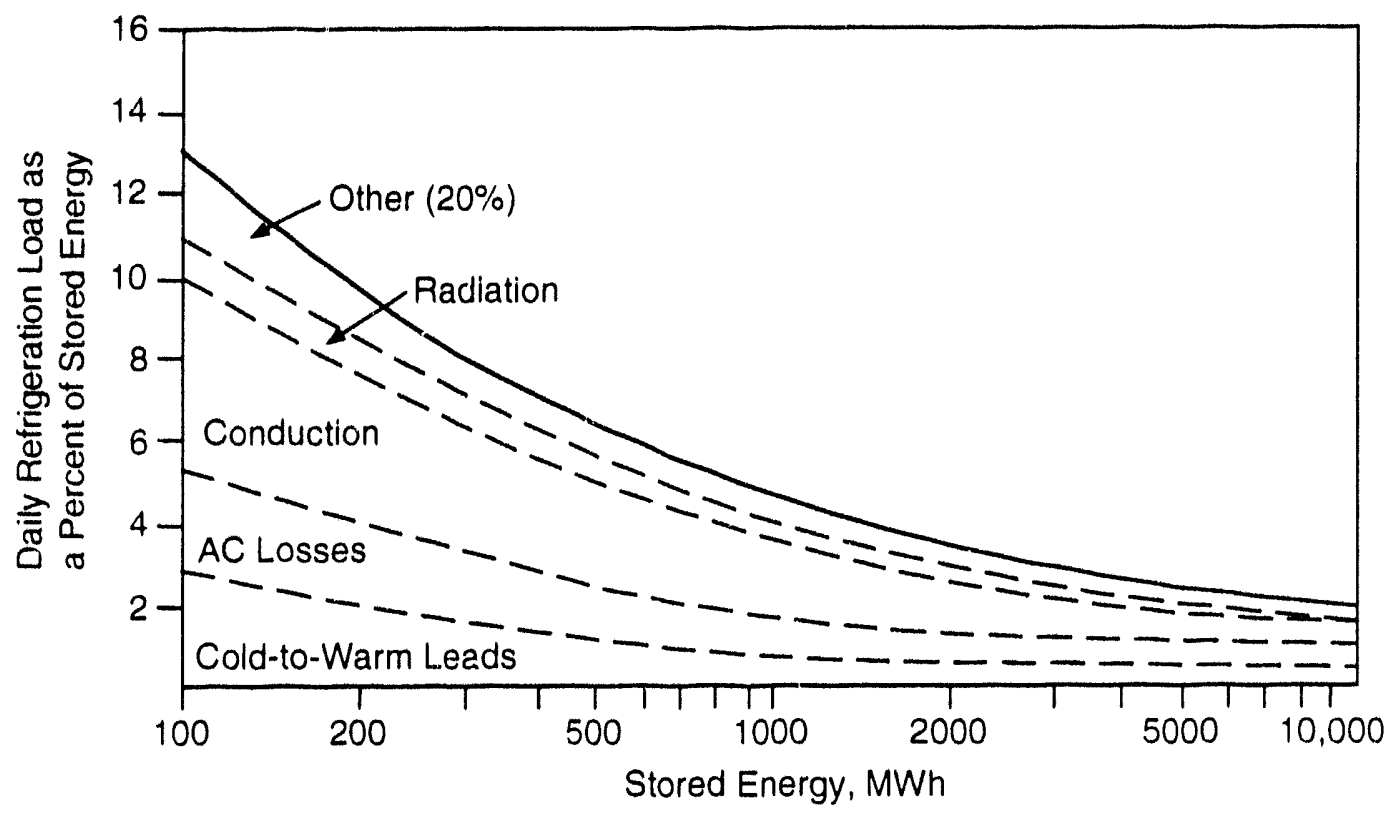

FIGURE 3-4 Components of Daily Refrigeration Load as a Function of SMES Stored Energy for SMES Coils Constructed in Competent Rock

a $10 \mathrm{MWh}$ SMES unit by up to 50\%. In addition, power consumption for refrigeration might be red sced by up to a factor of 50. This could greatly improve SMES efficiency, especially for smaller units.

Operating at $77 \mathrm{~K}$ could simplify SMES operation and increase reliability. In addition, cool-down time and the forces associated with cool down would be drastically reduced, and conductor stability would be greatly increased. However, should a fault develop, the slower propagation velocity of the normal zone could make protection of the SMES magnet much more difficult. In addition, the present brittleness of HTS could make dealing with the forces associated magnet energization and cool-down more difficult.

Presently, the cost of HTS is estimated to be about one order of magnitude more than NbTi conductors [10]. Although the likely future cost of HTS is not presently known, developers hope to achieve a cost $(\$ / \mathrm{kg})$ comparable to $\mathrm{NbTi}$. Most $\mathrm{J}_{\mathrm{c}}$ measurements of $\mathrm{HTS}$ at $77 \mathrm{~K}$ are made in self field. The $\mathrm{J}_{\mathrm{c}}$ in self field of a present HTS operating at $77 \mathrm{~K}$ is at least one order of magnitude less than that of an $\mathrm{NbTi}$ conductor operating at $4.2 \mathrm{~K}$; the performance ratio is even worse at SMES design operating fields of 5-6 T. Therefore, the cost of HTS in an SME is likely to be significantly higher than for the present NbTi conductor used in present SMES designs.

Although an all-HTS-based SMES design does not seem very promising at present, an HTS-based current lead could offer a significant reduction in the refrigeration load associated with the warm-to-cold leads in present SMES designs. Several groups have demonstrated that an HTSbased warm lead operating between $77 \mathrm{~K}$ and 4.2 or $1.8 \mathrm{~K}$ could reduce the refrigeration load 
associated with the warm-to-cold leads in present SMES designs by up to a factor of ten. Argonne National Laboratory is working with Superconductivity Inc. (SI) to develop HTS-based current leads for SI's $1 \mathrm{MWs}$, small-scale SMES designs being marketed to solve power quality problems (see Section 5). 


\section{Large-Scale SMES}

\subsection{Introduction}

Large-scale SMES has been under development since the 1960s, when the potential to store electrical energy in a large magnet was realized by researchers in Europe and the United States. In 1963, J. Stekly proposed a SMES design, and in 1970, M. Ferrier proposed using a single, large, centrally located SMES unit to satisfy all of Electricité de France's cyclic power needs.

The present-day, large-scale SMES, incorporating a multiphase Graetz bridge to provide the interface between a large magnet and the electric utility grid was proposed by Professors Peterson and Boom in 1971. In 1972, this prompted the U.S. Atomic Energy Commission to commission Los Alamos National Laboratory to determine whether or not such a concept would be economically justified and to create a program plan to develop the concept. The initial economic analysis showed that if cold structure was used to contain the large Lorentz forces associated with a SMES unit, the SMES unit would be too expensive to compete with existing storage options such as pumped hydro or conventional gas turbine generation. This led to development of the warm structure concept of using struts to transfer the Lorentz forces to free soil or rock.

Subsequently, Los Alamos was contracted by the U.S. Department of Energy to develop a reference design for a large-scale, low-aspect-ratio, 1,000 MWh SMES unit incorporating warm structure and built deep in soil. The study concluded that an economically competitive unit would be in the size range of 1,000 to 5,000 MWh [11]. In 1981, the Electric Power Research Institute (EPRI) funded Bechtel to evaluate SMES. This study concluded that a shallow design would be more cost-effective [12]. Subsequent work in 1984 and 1985, supported by DOE at EPRI, funded Bechtel to improve the design [7,9]. Figure 4-1 depicts a generic plan view of this concept, while Figure 4-2 shows the relative cost-effectiveness of a large-scale SMES unit for diurnal load leveling as a function of the number of hours per day of discharge capacity. The $y$-axis shows the revenue requirements for various SMES options, battery storage, underground pumped hydro storage, compressed-air energy storage (CAES), and a conventional gas turbine. All values are in $1985 \$ / \mathrm{kWh}$. The slope of each curve evaluated at $1 \mathrm{~h} / \mathrm{d}$ of operation is proportional to the powerrelated cost $(\$ / \mathrm{kW})$ of each technology.

As is evident in Figure 4-2, pumped hydro, CAES, and gas turbines all have high powerrelated costs relative to the PCSs used in battery storage and SMES. Thus, shorter peaking applications (a few hours of discharge per day) favor these storage options. The curves are asymptotically proporit nal to the energy-related costs $(\$ / \mathrm{kWh})$ of each technology for large hours of discharge per day. These curves illustrate that SMES and batteries have large energy-related costs relative to the other technologies (in the case of gas turbines, the energy-related cost is equal to the fuel cost to produce $1 \mathrm{kWh}$ of electricity). 


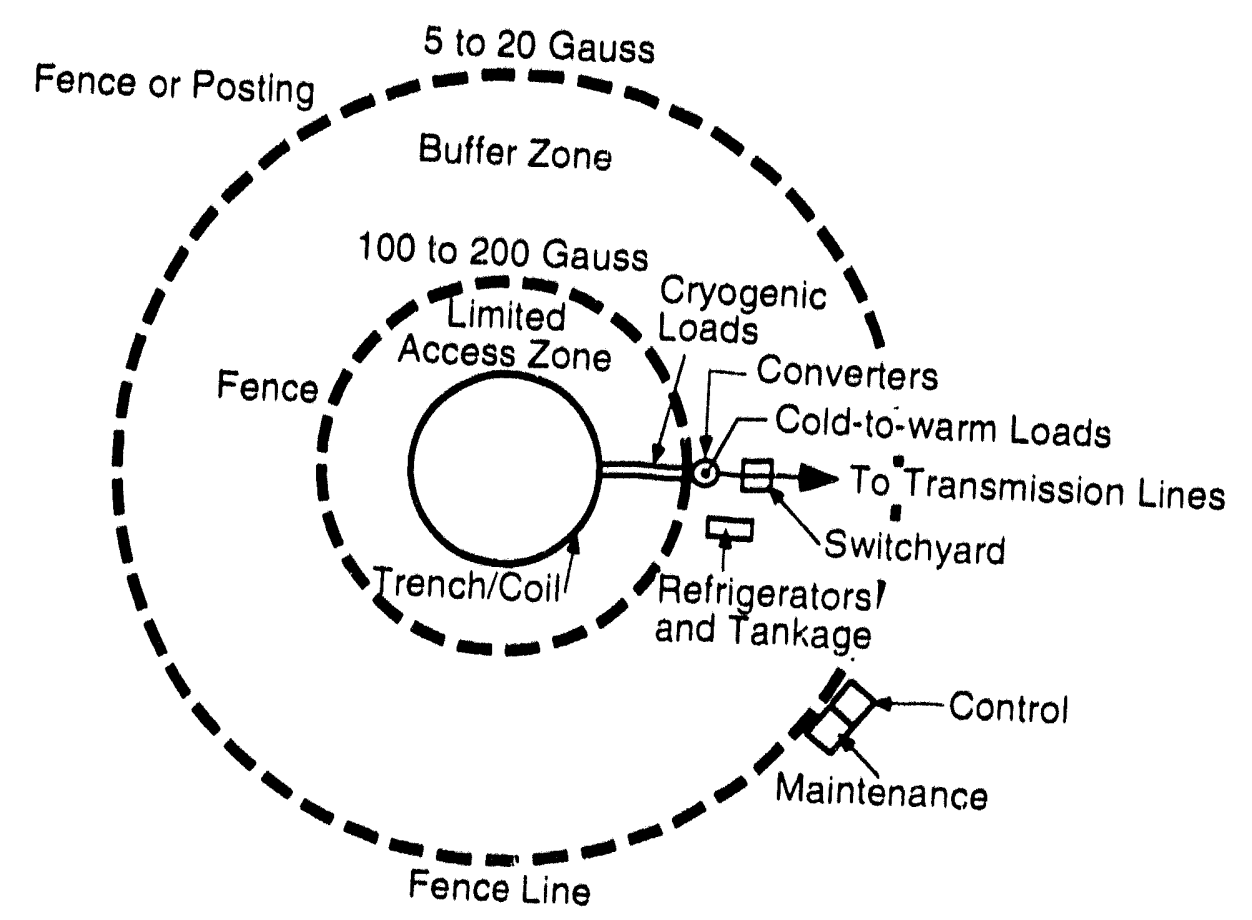

FIGURE 4-1 Artist's View of Generic SMES Plant

Figure 4-2 also illustrates the large economy of scale associated with SMES energy-related costs. This makes SMES cost-competitive out to more hours per day of operation as the storage while a 10,000 MWh SMES is cost-competitivetitive up to two hours per day of discharge, discharge). For the assumption used in thetive up to approximately six hours per day of constructed in soil has a lower revenue require analysis summarized in Figure 4-2, an SMES different set of assumptions, it is not clear whether than for one constructed in rock. Under a energy-related cost larger or smaller than a comparable SMES constructed in soil will have an the United States, there are more sites suitable for conste SMES constructed in rock. However, in

The analysis above includes only the SMES benefits attributable to diurnal load leveling In addition, because of the ability of an SMES to respond very rapidly to changing load conditions (an SMES can switch from full charge to full discharge in about two cycles), an SMES unit can Satisfy spinning reserve, dynamic response, and black start-up roles. In principle, a battery with a chemical reaction, it can not sustain a rapid change a battery derives its electrical energy from a an SMES unit should not degrade with repange in power level. Furthermore, unlike batteries, should be low. In principle, a flywheel SES couted cycling, and operation and maintenance costs more cost-effective than an SMES at smaller sizes. 


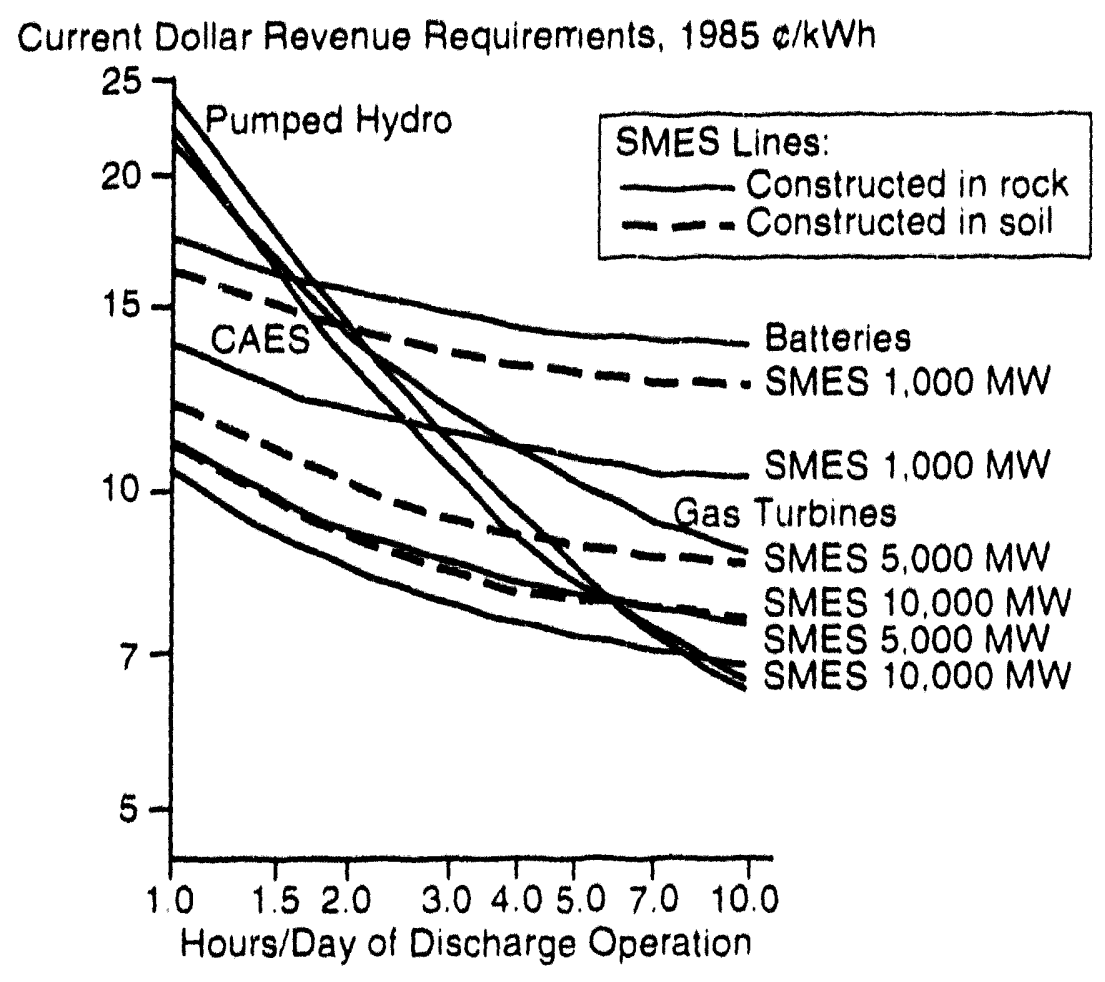

FIGURE 4-2 Relative Cost-Effectiveness of Large-Scale SMES for Diurnal Load Leveling as a Function of Daily Discharge Period

In parallel with the above analytical effort. Los Alamos built and installed a 30 MJ SMISS unit on the Bonneville Power Authority (BPA). This unit was successfully operated to stabilize at large. long-distance ac transmission operating between BPA in the Pacific Northwest and Los Angeles. In 1986, Japan performed an analysis comparable to the Bechtel study. except for a 5,000 MWh SMES built in rock. In 1986. EPRI proposed building a 10 MWh Engincering Test Model. In 1987, the Strategic Defense Initiative Office (SDIO) and EPRI initialted work to design and build a $20 \mathrm{MWh}$ Engineering Test Model (ETM). So far, two designs have been developed and costed, but the selection of which design to build and the decision of whether to go ahead with construction of the ETM have not been made.

To date, most SMES development work has consisted of engineering design and economic feasibility analyses in the United States and Japan. With the exception of two small-scale units having a storage capacity of several MJs, no large-scale SMES device has been built. Table +-1 summarizes several small SMES units that have been built, while Table 4-2 summarizes proposed SMES devices for which de: 'wh studies are in progress or construction has begun. 
TABLE 4.1 Summary of SMES Units Built [13]

\begin{tabular}{lccccc}
\hline Country & $\begin{array}{c}\text { Stored } \\
\text { Energy }\end{array}$ & $\begin{array}{c}\text { Power } \\
\text { Rating }\end{array}$ & $\begin{array}{c}\text { Time } \\
(\mathrm{s})\end{array}$ & Comments \\
\hline U.S. & $8.4 \mathrm{kWh}$ & $10 \mathrm{MW}$ & 3 & Used to stabilize Pacific Intertie 1983-1984 \\
Japan & $3.280 \mathrm{Wh}$ & $5.70 \mathrm{~kW}$ & 0.3 .40 & Verification of SMES pertormance and control \\
\hline
\end{tabular}

TABLE 4-2 Ongoing SMES Design/Cost Studles and SMES Construction

\begin{tabular}{|c|c|c|c|c|}
\hline Country & $\begin{array}{l}\text { Store } \\
\text { Energy }\end{array}$ & Power Rating & $\underset{(s)}{\operatorname{Tim} \theta}$ & Comments \\
\hline U.S. & $20 \mathrm{MWh}$ & $20 / 400 \mathrm{MW}$ & $7200 / 100$ & Engineering test model \\
\hline Germany & $\begin{array}{l}0.44 \mathrm{kWh} \\
0.08 \mathrm{kWh}\end{array}$ & $\begin{array}{l}1 \mathrm{MW} \\
0.25 \mathrm{MW}\end{array}$ & $\begin{array}{l}3 \\
1\end{array}$ & $\begin{array}{l}\text { Test load response, } \\
\text { compensation of load fluctuations }\end{array}$ \\
\hline Japan & $\begin{array}{l}700 \mathrm{Wh} \\
100 \mathrm{kWh}\end{array}$ & $20 \mathrm{~kW}$ & 126 & $\begin{array}{l}\text { Study cooling/stability issues, } \\
\text { SMES demonstration }\end{array}$ \\
\hline Former USSR & $28 \mathrm{kWh}$ & $20 \mathrm{MW}$ & 5 & Test on Moscow grid \\
\hline
\end{tabular}

\subsection{In-Ground SMES Designs}

Three in-ground SMES designs have evolved: a trench design for construction in soil and two tunnel designs for construction in rock. In 1979 Los Alamos proposed a single-tunnel, inrock design for a large-scale SMES. This design used a solenoid with a $\beta$ of $0.1-0.2$. Subsequently, Los Alamos developed another design for locating the SMES coil in open trench built in soil. Because the maximum working stress in soil is approximately a factor of four less than in rock, working stress must be reduced. To accomplish this, the Los Alamos design calls for a somewhat lower magnetic field and uses a lower aspect ratio $(\beta=0.01)$ for the solenoidal coil. As shown in Figure 4-3, for a given storage capacity, the SMES built in soil is approximately twice as big in diameter as the comparable SMES unit built in rock. In addition, the fringe field for the trench design extends far beyond that for the rock design.

The primary advantage of the in-soil design is that acceptable sites are readily available. In contrast, an in-rock design permits construction only in competent rock which greatly limits site 


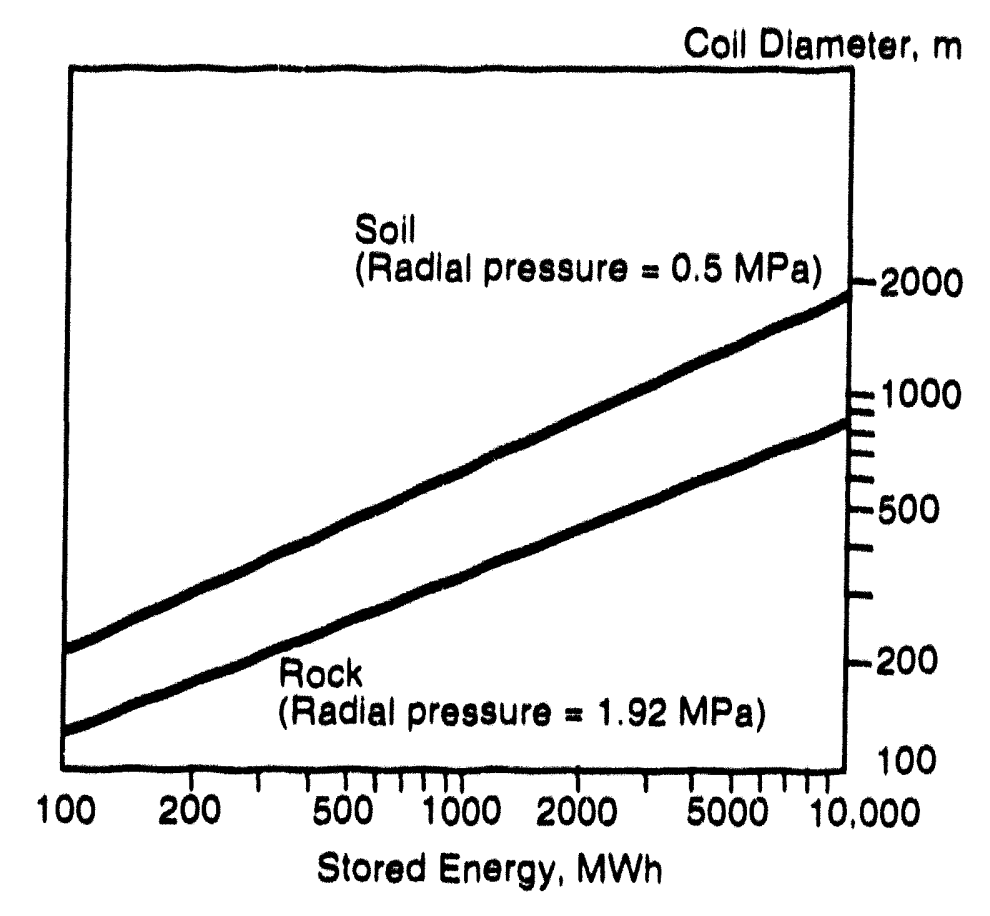

FIGURE 4.3 SMES Coll Diameter for Construction in Soll and Rock versus SMES Stored Energy

availability. In the United States, the greater availability of in-soil sites more than offsets the corresponding larger SMES dimensions. In contrast, in Japan, the premium placed on land favors an in-rock design. The Japanese have developed a 3-tunnel, in-rock design employing a $\beta$ of 0.1 .

Figure 4-4 shows an artist's drawing of the in-soil trench design for a large-scale SMES. The trench is dug at surface level, load-bearing, reinforced-concrete columns provide structural support. Upon completion of the SMES unit, a concrete roof is used to enclose the trench. A carbon-steel vacuum line is used to seal the cavity.

Preliminary design and cost studies have been performed for a 5,000 $\mathrm{MWh} / 1,000 \mathrm{MW}$ SMES unit for both the American in-soil, trench design and the Japanese, 3-tunnel, in-rock designs. Table 4.3 summarizes the results of these studies.

The Japanese design appears to be driven by two concerns: (1) the high cost of land in Japan, and (2) the high cost of helium in Japan. The higher allowed working stress of rock versus soil permits use of a higher magnetic field and a more optimal $\beta$. Consequently, the radius and aspect ratio for the American and Japanese designs differ by a factor of 5 and result in the Japanese design having a factor of 25 smaller footprint and having approximately a factor of 5 smaller coil volume. To operate at a higher magnetic field, the Japanese design employs a 


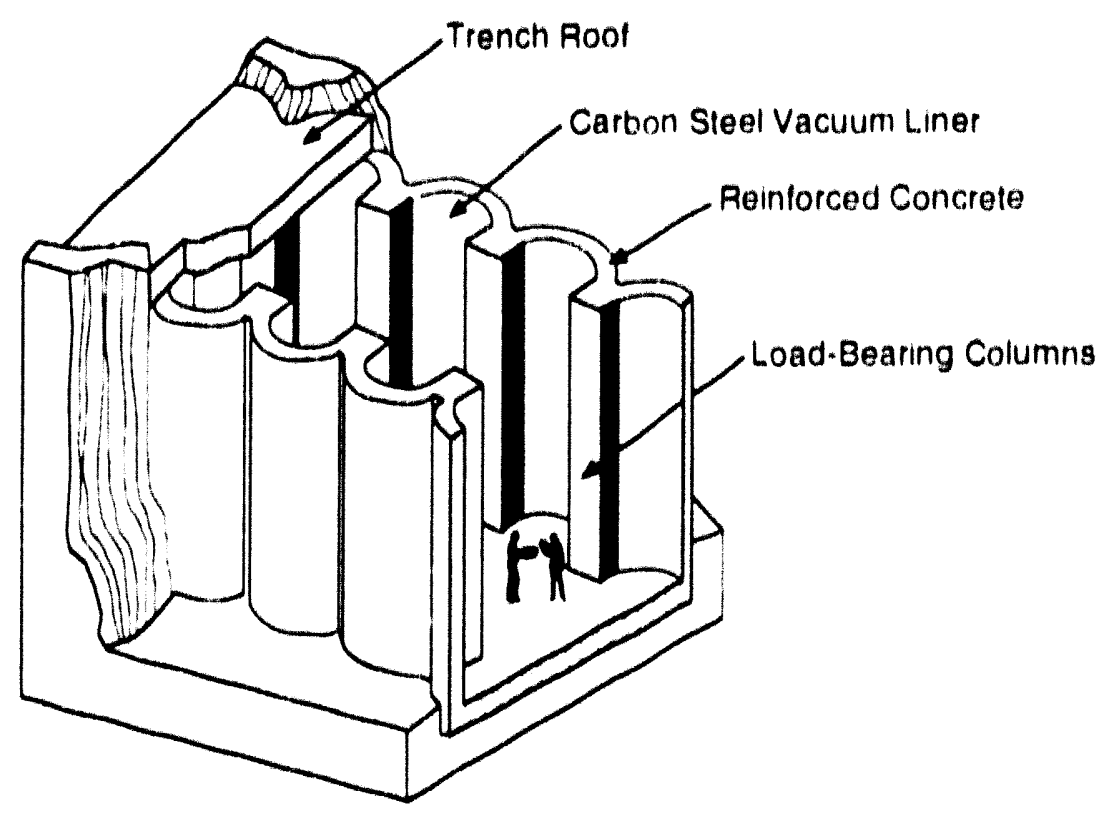

FIGURE 4.4 Artist's View of Trench Construction for In-Soil SMES Unit

TABLE 4.3 Comparison of Japanese and American Large-Scale SMES Designs

\begin{tabular}{lll}
\multicolumn{1}{c}{ SMES Parameter } & \multicolumn{1}{c}{ Japan } & \multicolumn{1}{c}{$\begin{array}{l}\text { United } \\
\text { States }\end{array}$} \\
& & \\
Configuration/Medium & 3. tunnelirock & Irench/soil \\
Diameter (m) & 200 & 1000 \\
Aspect Ratio & 0.1 & 0.02 \\
Maximum Field (T) & 8.4 & 5.0 \\
Maximum Current (KA) & 707 & 200 \\
Type of Superconductor & $\mathrm{Nb}_{3} \mathrm{Sn}$ & $\mathrm{NbTi}$ \\
Operating Temperalure (K) & 4.2 & 1.8 \\
Round-Trip Elficiency (\%) & 90 & 92 \\
Total Cost (Billions of 1985 U.S. Dollars) & 1.5 & 1.0
\end{tabular}


$\mathrm{Nb}_{3} \mathrm{Sn}$ conductor which operates at the traditional 4.2 K. Although $\mathrm{Nb}_{3} \mathrm{Sn}$ conductor is more costly than NhTi conductor, operating closer to the optimum $\beta$ and at a higher field reduces the required IS by approximately $30 \%$.

The American design calls for the conductor to carry a very large current: the current flowing in the Japanese design is enormous. This means that coil protection, should a normal zone develop, is a major concem. One technique proposed Io prevent coil meltdown is to forcibly dump all the liquid helium when the formation of a normal cone within the coil is detected (a voltage develops across the coil). This would quickly drise the entire coil normal, thereby spreading energy dissipation throughout the coil. In addition. the conductor would be imledded in a mussive amount of high-purity aluminum. which would provide an alternative current path and enough thermal mass to prevent an excessive temperature rise within the coil assembly. Demonstratton of adequate coil protection is once of the goals of the ETM project.

Both designs predict a very high $(>=90 \%)$ round trip efficiency. Approximately $5 \%$ of the loss is expected to occur within the PCS: the remainder is associated with the refrigerattion power needed to maintain superconductivity. Both designs have a cost in excess of one hilliun 198.5 U.S. dollars. Although the Japanese design is estimated to cost substantially more than the American design, it is not clear whether or not the difierence is due to the use of different designs. different construction practices in each country, and/or the difficulty in converting costs from yen to dollars. $" 1$ In either case, a large-scale i VIES device is a very expensive item, comparable in cost to a $1.000 \mathrm{MW}$ nuclear power plant.

\subsection{Engineering Test Model}

The Engineering Test Model Project is being funded jointly by the Defiense Nuclear Agency and EPRI. The ETM is being designed to satisfy (wo missions: (1) diumal load leveling (10 MW for 2 hours. 20 MWh) and (2) power source for a free-electron laser in suppon of SDIO $(400 \mathrm{MW}$ for $100 \mathrm{~s}, 11.1 \mathrm{MWh}$ ). Two competing teams have been formed to design and huild the ETM. The first team is headed by Bechtel and includes General Electric, General Dynamics, and General Atomics. The second team is headed by Ebasco and includes Westinghousc Electric and the University of Wisconsin. Although a 20 MWh SMES unit is too small to be economically justified for providing diurnal load leveling, the unit has the same magnitude of current llowing through the coil and generates the same magnetic field as the full-scale design. Hence, the unit can he used to test and validate all the concepts proposed for a full-scale SMES unit. Consequently. scale up from the ETM to a full-scale SMES unit is not expected to pose any new problems.

Five sites have been identified for the ETM: (1) Badger Site in Wisconsin. (2) Hanover Site at BPA in Washington, (3) White Sands Missile Range Site in New Mexico, (4) Orogrande Site in New Mexico, and (5) Monahans Site in Texas. An Environmental Impact Statcment is

11 The cost assumptions for the Japanese and American designs differ. Dr. Hassemzahl reestimated the cust fir the Japanese design at 3 billion dollars using U.S. cost rules. 
being prepared for eacis site. The environmental studies concluded that, unlike many large construction projects, the SMES used in the ETM is environmentally benign. The ETM should produce no hazardous wastes, air or waller pollution, noise, or radical changes fo the landscape

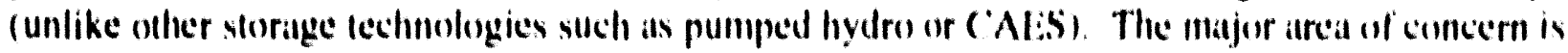
stray magnetic lields. The ETM will produce a background magnetic ficld equal to the earth's magnetic field (0.5 gauss) at a horizontal distance of about $8(x)$ in from the center of the soil and all a vertical distance of about $1,(00) \mathrm{m}$ above the eoil. When operating as diumal storage, the L:TM will produce an essentially de magnetic ficld. However. when providing a pulse of energy to the free electron laser, significant ac magnetic fields will be produced. (iiven the present concern related to electromagnetic fields produced by high-voltage transmission and distribution lines. magnetic fields prisluced by the ETM could raise a public concem.

Except for the conductor asiembly. the Iwo contractors have developed similar desiens for the ETM. Buth use a low-aspect-ratio solenoid. huried in an open trench in soil. Detailed desighs have been completed and costed, and both conductur assemblies have been validated. On lhe hasis of the cost models developed for the ETM, the Iwo contractors independenily estimalle thit the capital cost for a $5(0)()$ MWh SMLS unit may he higher than in previous estimates. 1: Presently. the cost and schedule: details are being relined.

Table $4-4$ summarizes key aspects for each design. The Bechtel design calls for a coil current of $2(x) \mathrm{kA}$ (similar to the original Bechecl design). A cable :in-conduit conductor with a hollow core is used. Figure 4.5 shows a cross section for the Bechel $200 \mathrm{k} A$ conductor. The Ehasco design uses a monolithic conductor consisting of a high-purity aluminum stabiliaer containing eight NhTi conductors each in a copper matrix as shown in ligure t.6. The labasco design requires the eonductor assemble 10 carry $50 \mathrm{k} \Lambda$.

TABLE 4.4 Summary of Bechtel and Ebasco ETM Designs

\begin{tabular}{llc}
\multicolumn{1}{c}{ Parameter } & Bechtel & Ebasco \\
Energy (MWh) & 20.4 & 21 \\
$I_{\max (K A)}$ & 200 & 50 \\
Inductance (L) & 367 & 60.5 \\
Diameter (m) & 129 & -100
\end{tabular}

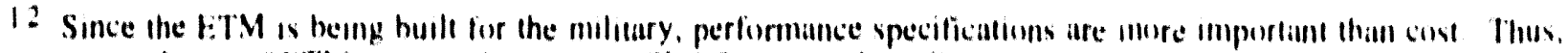
extrapulation of I:TM cost to a larger scale SMI:S may not be vallid 


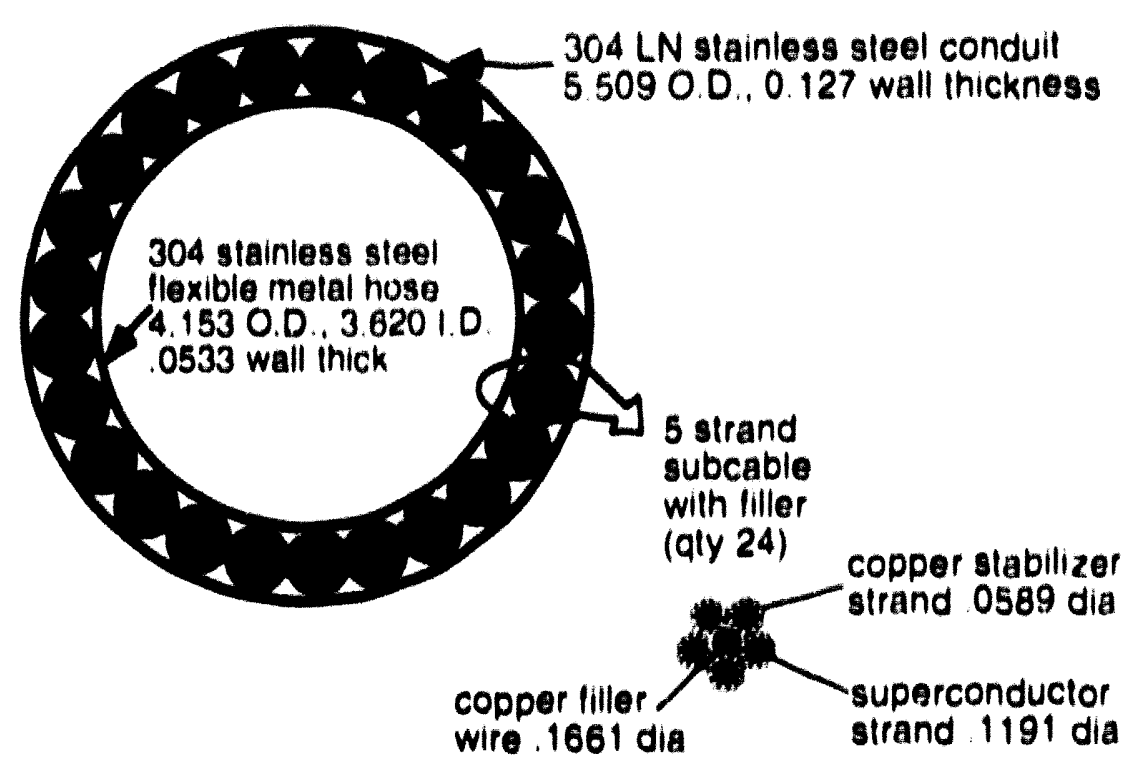

FIQURE 4.5 Cross Section lor Bechtel $200 \mathrm{kA}$ Conductor for ETM

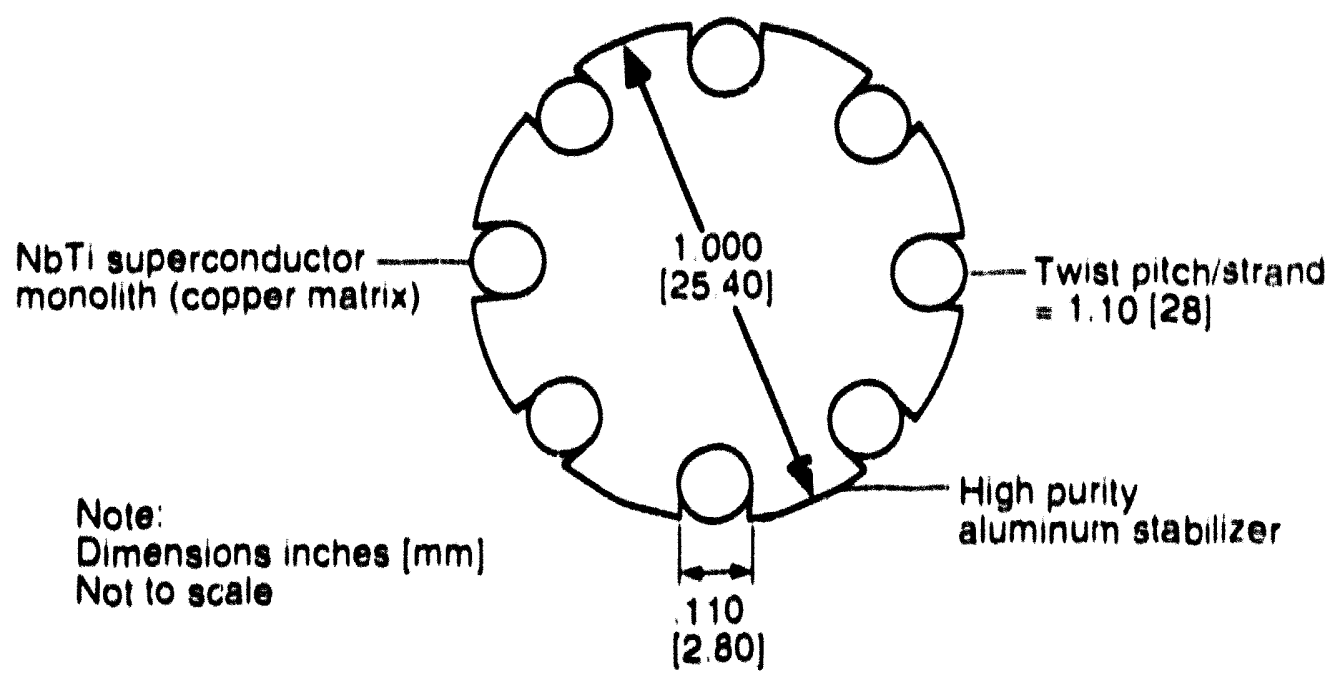

FIGUAE 4.6 Cross Section for Ebasco 50 kA Conductor for ETM 
An SMES Utility Interest Group has been formed by EPRI. Initially, the group involved the five utilities bidding to host the ETM and the two design teams. The sole purpose of the group was to promote the ETM project. Meetings were held twice per year. Subsequently, the group has expanded to include other SMES applications: the membership has been expanded to include utilities, contractors, suppliers, vendors, and researchers, Seventy-live members altended the June 1992 meeting to discuss a wide range of SMES-related issues.

Presently, the outlook for actual construction of the ETM is ulicertain. Because of the conclusion of the cold war, the Defense Nuclear Agency is on record as no longer needing the ETM because the free electron laser project is no longer going firward. However, in the past. SMES has enjoyed much congressional support which has allowed the SMISS program to survive previous altempes fo cuncel funding. 


\section{Small-Scale SMES}

\subsection{Introduction}

With the increasing use of electronic equipment. power quality and custom power issues are gaining importance in the delivery of electric power. Electronic equipment such as computers. electronic processing controls. and variable-sped motor controllers call be quite sensitive to voltage sags and momentary loss of power.

Voltage sags can be caused by lightning strikes and routine switching thal takes place on transmission and distribution systems. As utilities become more inlereonnected, the exlent of the problem grows. However, these conditions unly oceur for brief periods. Approximately 85.9(1) of the momentary loss of power or voltage sag events persist for 5-30 eycles (less than one hall second). A small-scale SMES. Mywheel, or possibly ballery system together with a PCS could eliminate the consequences of such events. An article in Business Week $|1+|$ estimated such power conditions are costing industry in the United States 26 hillion dollars per year. However. the present lack of standards is inhibiting defining and solving this issue.

One standard that has been developed is the CBEMA voltage standard for mainframe computer systems. This standard is illustrated in Figure 5-1. The upper eurve shows the maximum voltage a compuler must he designed to withstand for various durations Thus, a mainframe should be able to tolerate a $6 \%$ over voltage indefinitely and a $15 \%$ over voltage for I eycle. If the voltage exceeds these limits, voltage breakdown within the equipment might oceur. Conversely, the machine should be designed to operate at $1.3 \%$ under voltage indefinitely and at $30 \%$ under voltage for I eycle. If the voltage is less than these limits. the computer power supply might not have enough stored energy to maintain the desired internal voltage levels. Although mainframe computers are usually designed to meet the CBEMA standard, no comparahle standard exists for other equipment. Thus, when a problem occurs, there is no means available (o determune whether the fault lies with the utility, customer, or eyuipment minufiaturer.

\subsection{Superconductivity Incorporated}

A director of electric marketing and customer service in the Wisconsin Power and Light Company observed the growing concern of commereial and industrial customers with power quality-related issues and started a company named Superconductivity Incorporated (SI) ${ }^{13}$ (1) develup a solution (o) the problem.

13 For more information on SI. see the Directory of Ceramic Superconductor Research and Related Reseirch Bearing Upon the Electric Power Secoror. 


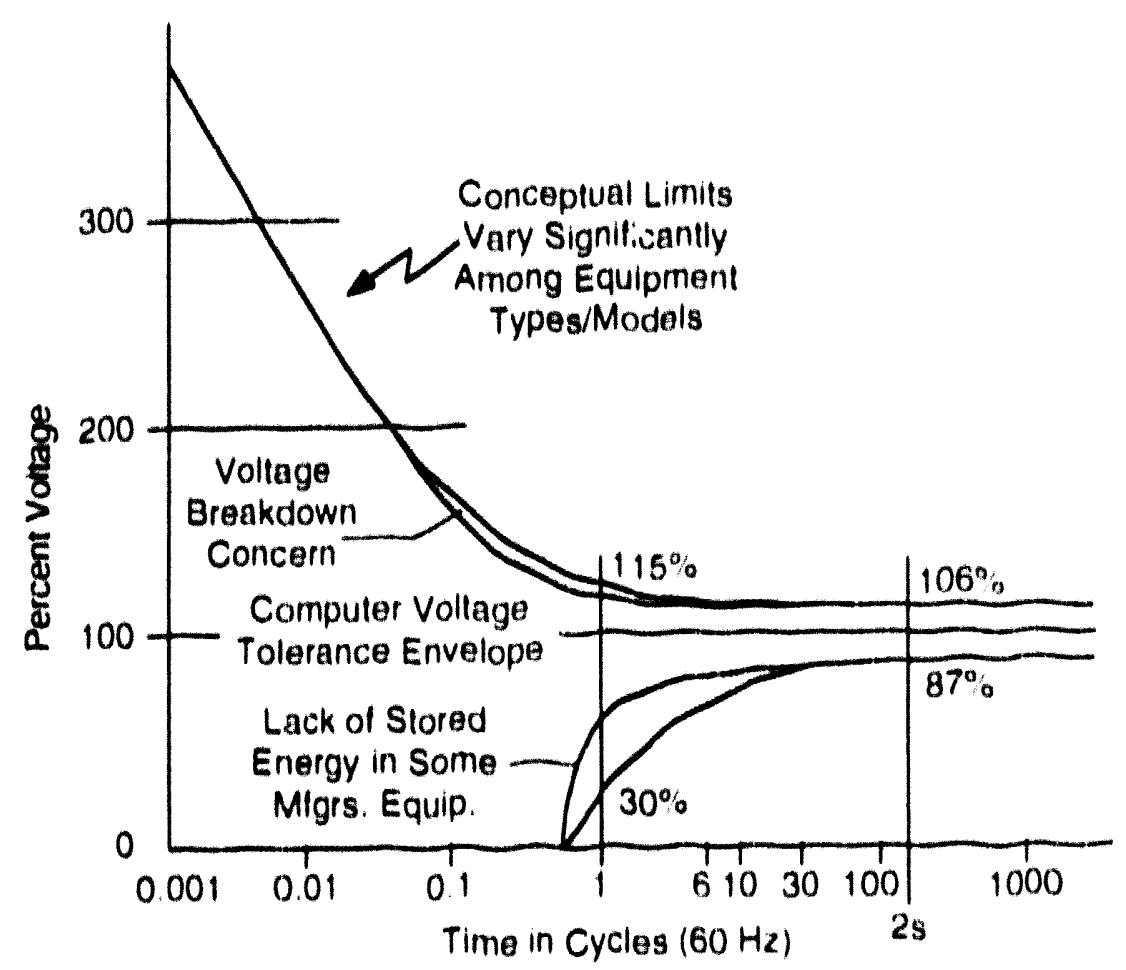

FIGURE 5.1 CBEMA Voltage Standard for Maintrame Computers

\subsubsection{Superconducting Storage Device}

A small-sciale, L.TS-hatsed SMES unil developed by SI has a rating of I MJ of ellergy capacity and I MW of power |15|. Because discharge oceurs in a very short period of lime (less than a second), ac losses within the superconductor are a much bigger concern than in a latrge-sciale SMES unit. ${ }^{1.1}$

The SMESS unit consists of a solenoid made from low-ile-loss, multililamentary Nhli wire. It employs cold structure and incorporates a I MW PCS provided hy Asea Brown Boveri (ABB). The device provides up lo several seconds of backup power to the prosected load during vollage sags or momentary power losses. Figure 5-2 shows a cutaway drawing of the magnel and cryostill assembly for Sl's commerciat I MJ Supereonducting Storage Device (SSD) system. The magnet assembly is immersed in a pool of liquid helium and is connected to the PCS via a pair of current leads. The magnet is in the form of a solenoid and carries a current of helween $1 .(0)()($ alld

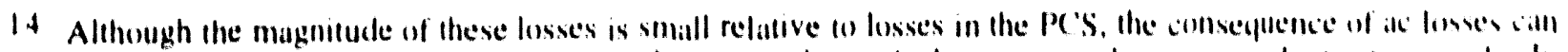

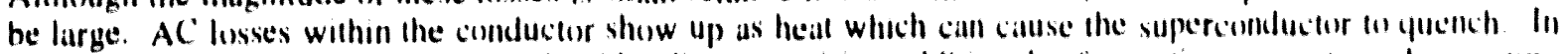
addition, the heat is deposited in the liquid helium. requiring additional refrigeration capacily and operating expense. 


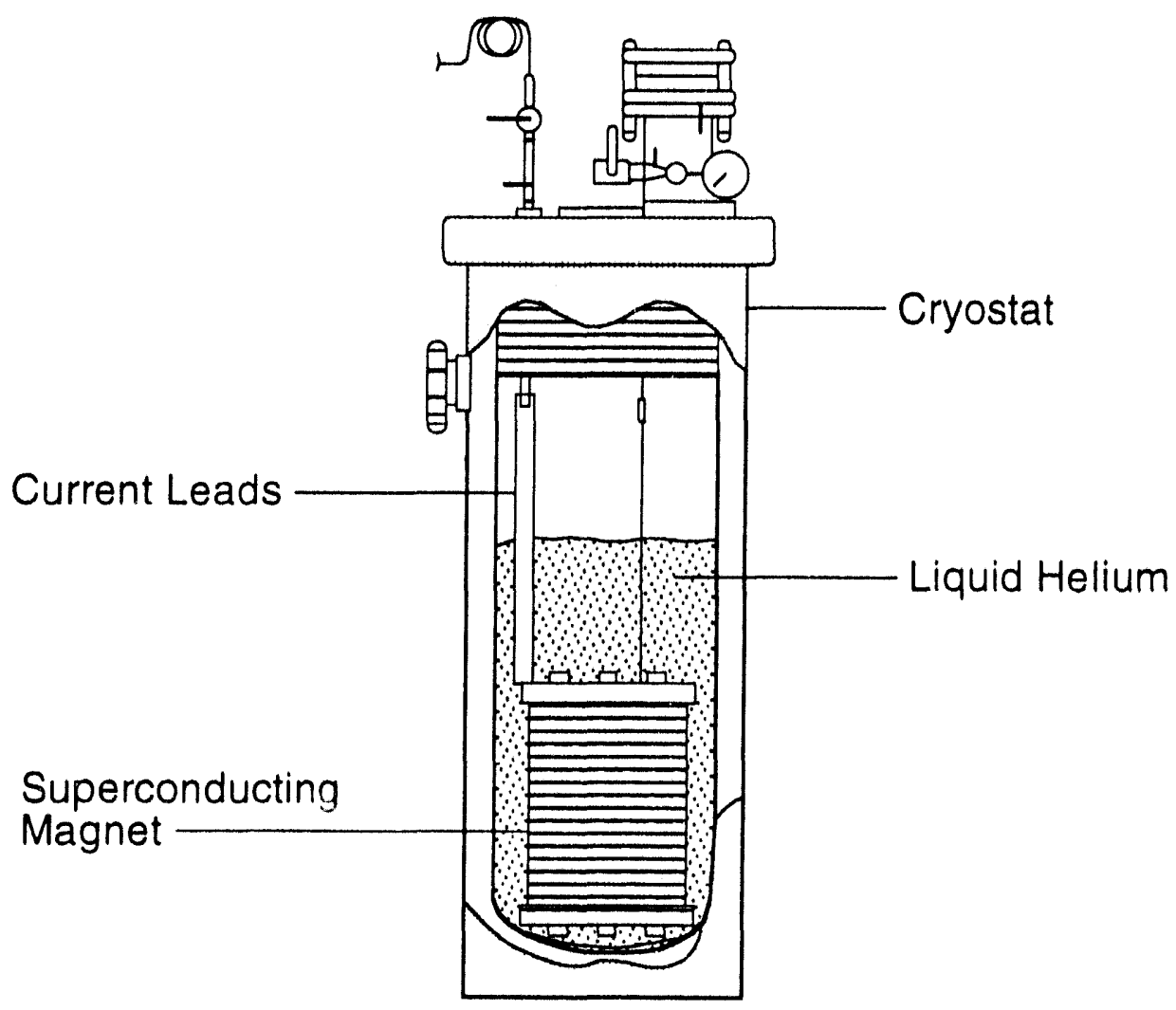

FIGURE 5-2 Cutaway Drawing of Magnet and Cryostat Assembly for Superconductivity Incorporates $1 \mathrm{MJ}$ Superconducting Storage Device

$1,500 \mathrm{~A}$. The wlenoid is $89 \mathrm{~cm}$ long and $46 \mathrm{~cm}$ in diameter and is housed in a cryostat that is $203 \mathrm{~cm}$ long with an outside diameter of $76 \mathrm{~cm}$. The relatively large cryostat provides a large reservoir of liquid helium to carry over operation of the SSD should the refrigerator fail.

Figure 5-3 shows a block diagram for the complete system. The system consists " , solid-state isolation switch, transformer, inverter, voltage regulator, magnet, magnet power supply, refrigerator, and SSD system control. Under normal conditions, the utility grid feeds the magnet power supply, which charges the magnet. Charging occurs over a period of several minutes to prevent overload of the utility grid. The magnet current circulates through the magnet and the normally closed switch $S$ in the voltage regulator. The load is connected directly to the utility grid, and the SSD is isolated from the load (should something happen to the SSD system, the load is protected).

When a voltage sag or loss of power is detected, the solid-state isolation switch disconnects the load from the utility grid and switches in the SSD. The SSD controller begins discharge of the magnet. Switch $S$ opens, causing current from the magnet to charge the capacitor across the inverter. When the voltage reaches a preset level, switch $S$ closes. The inverter converts this 


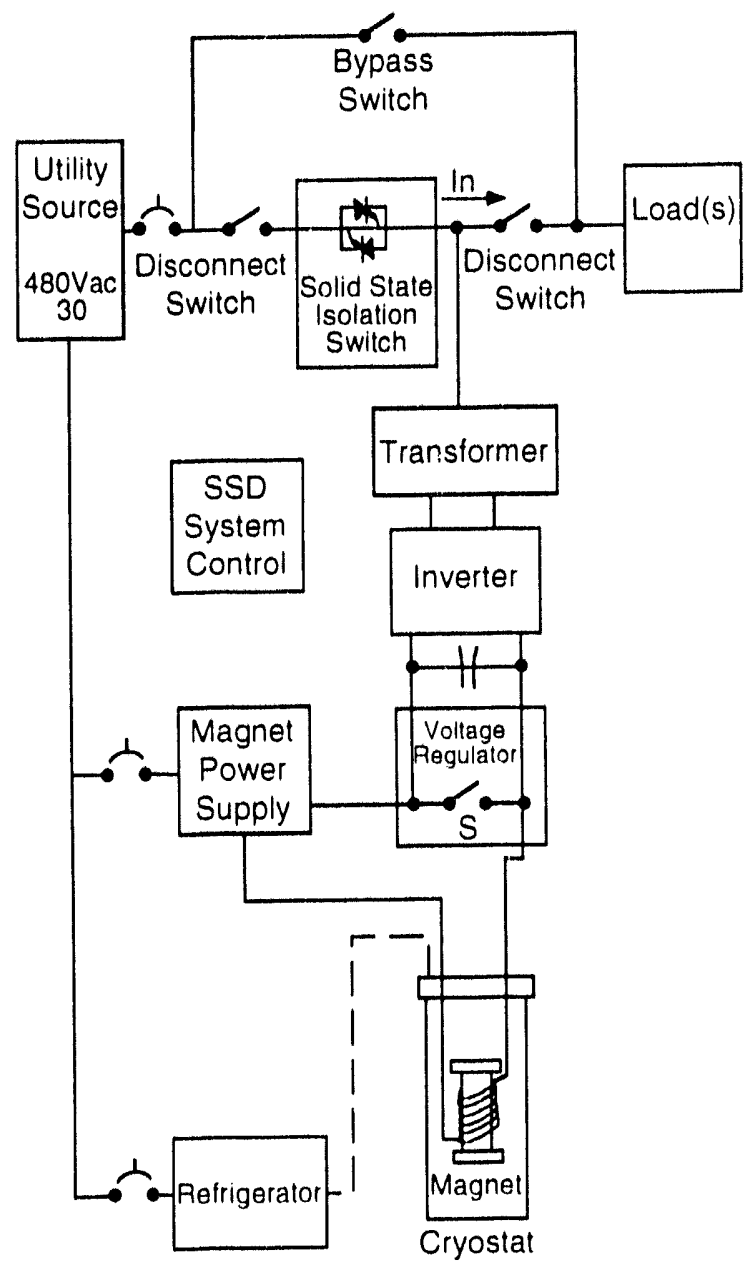

FIGURE 5-3 Block Diagram of the Superconductivity Incorporated $1 \mathrm{MJ}$ Superconducting Storage Device

energy to ac power causing the capacitor to discharge. When the voltage reaches a preset minimum value, switch $\mathrm{S}$ opens, and the cycle begins again. Essentially, energy is withdrawn from the magnet in the form of current pulses. As the magnet discharges, the duration of the pulses increases to maintain a constant power level, while the capacitor across the inverter maintains a constant voltage during discharge. The inverter converts the dc voltage across the capacitor to a 60 -cycle ac voltage. A transformer is used to match the ac voltage to the line voltage. Upon resumption of normal power conditions, the solid-state isolation switch reconnects the load to the grid, disconnects the SSD from the load, and begins recharging the magnet. Because the entire system operates at electronic speeds, switch over to the SSD begins within $450 \mu \mathrm{s}$. Full power is reached in 2-4 ms (a fraction of a cycle).

One of SI's innovations is to package the entire system as a black box as far as the user is concerned. The entire system is delivered inside a tractor trailer which is delivered to the on-site location. This provides a compact, mobile system with only two electrical connections to the 
outside world. In addition, SI provides all maintenance for the SSD system. Recent versions of the system contain a supply of up to 60 hours of liquid helium. The system is monitored remotely at SI's Wisconsin headquarters. Should a fault occur in the helium system, SI boards a plane, flies to the site, and repairs the unit without even involving the customer. Consequently, the customer is never made aware of the liquid-helium nature of the device.

The present system has several drawbacks, which SI is working to eliminate. First, the present cryostat has a very conservative and simple design; consequently, it has a fairly large room-temperature refrigeration load of $35 \mathrm{~kW}$. SI is designing a heat shield to improve cryostat performance and is working with Argonne National Laboratory to develop HTS-based current leads. These two changes should permit SI to install a smaller refrigerator or retain the present refrigerator and provide extended helium reserves. Second, the magnet current normally flows through the solid-state switches resulting in continuous losses of approximately $4 \mathrm{~kW}$. A persistent switch might reduce this load. Another big load comes from the conventional airconditioner used to remove heat generated by the PCS and other electronics from the trailer. Although refrigeration, both cryogenic and conventional space conditioning, contributes to a fairly high operating cost, the SSD is economically justified because it prevents costly outages. SI is working on reducing operating costs, but, in most cases, operating costs are not a crucial issue.

\subsubsection{Case Studies}

The initial design work for the SSD was performed during 1988 and 1989. Subsequently, the SSD unit has undergone extensive testing at various utility sites, starting in the summer of 1990. A $460 \mathrm{kVA}$ prototype unit was tested and used for demonstrations by Iowa Public Service Corporation, Wisconsin Public Service Corporation, Pacific Gas and Electric, and Puget Sound Power and Light Company. Establishing the correct control strategy for an SSD is important. If the SSD unit activates too early, it could trigger on false events and run out of energy during an actual event. If the SSD unit activates too late, it might not provide adequate carryover during switch over. Consequently, a different control strategy must be developed for each site. After initial shakedown to establish the best control strategy, most problems that occur are related to the PCS system. According to SI, there have been no failures of magnet-related components in any SSD unit to date.

\section{Georgia-Pacific Paper Mill in Bellingham, Washington}

This «nit was upgraded to provide $730 \mathrm{kVA}$ and was attached to a set of solid-state, variable-speed-drive motor controllers at a Georgia-Pacific paper mill in Bellingham, Washington during summer 1991. These motor controllers would shut down the motor during voltage sags or momentary power outages causing paper to cascade all over the plant floor. The SSD unit was connected in-series between the grid and the motor controller to provide a source of uninterrupted power to the motor controller. The device worked successfully, but was removed upon completion of the testing phase. To prevent possible failure of an SSD unit from interrupting 
service, subsequent SSD designs call for the SSD unit to be installed in parallel with the utility grid, as shown in Figure 5-2.

\section{Bay Area Rapid Transit in San Francisco, California}

An SSD unit was installed and extensively tested at Pacific Gas and Electric's (PG\&E's) test facility. On the basis of these tests, the use of an SSD is being examined to solve a loss-ofpower problem due to voltage sags on the Bay-Area-Rapid-Transit (BART) system. PG\&E has issued a Request for Proposal to solve the voltage sag problem on BART at two sites: (1) a site unconstrained by availability of space and power, and (2) a site within the tube that goes under the San Francisco Bay. Three SMES proposals (including one from SI), one battery proposal, and one proposal to use pulsed-duty rectifiers were received. The proposals have been evaluated and a final report is being prepared. According to Melissa Reading at PG\&E, the footprint for the battery system is much larger than the footprint for the SMES systems. However, the SMES systems cost more. The report will be published by PG\&E and EPRI and should be out during fall 1993. PG\&E hopes to begin testing, probably of an SI SMES unit, during 1994.

\section{IBM East Fishkill Facility in Hopewell Junction. New York}

The first commercial SSD unit was leased by Central Hudson Gas and Electric and was installed at the IBM East Fishkill Facility in Hopewell Junction, New York during spring 1992. The particular building that the SSD was attached to contained a megatester unit, used to perform accelerated lifetime tests on Very-Large-Scale-Integrated (VLSI) circuits. The megatester was very sensitive to voltage sags below $85 \%$ of nominal voltage. A voltage sag would cause the megatester to shut down, invalidating the test and, in many cases, causing damage to both the megatester and VLSI circuit under test. Consequently, voltage sags were quite costly. This prompted Central Hudson Gas and Electric to work with IBM to solve the problem.

It was specified by IBM that the solution should be mobile because chip testers are frequently moved from one site to another. They also required a moderately compact solution ruling out installation of an Uninterrupted Power Supply (UPS). The use of batteries would require construction of a costly addition to the existing building to house the batteries. Furthermore, IBM was interested in a research and development (R\&D) type project. Consequently, a $750 \mathrm{kVA}$ SSD unit was selected.

Between June and November of 1992, the SSD unit successfully provided ride-through of 20 events. During a February, 1993 ice storm, the SSD prevented 10 voltage sags in one day. The SSD system consumes approximately $41,614 \mathrm{kWh}$ of real power and $33,608 \mathrm{kVarh}$ of reactive power per month. Monthly electric bills have averaged $\$ 2,500$ ), about what a UPS would cost. Although IBM and Central Hudson Gas and Electric would like to reduce operating costs, they have expressed overall satisfaction with the performance of the installed SSD unit and they

have even considered adding a second unit. 
Sonoco Plastic Bag Extrusion Plant on Carolina Power and Light System

An SSD unit was installed in March, 1993 in a Sonoco plastic bag extrusion plant located in Carolina Power and Light's service area. This plant contains $36 \mathrm{dc}$ extruders, winders, and controls [16]. The de drives shut down when the voltage falls to $88 \%$ of nominal, causing the plastic to freeze. The company estimates that each outage costs approximately $\$ 10,000$, and there are $20-25$ outages per year.

The bag plant facility provides new challenges to operation of the SSD unit. In particular, the dc drivers produce lots of harmonics, which could cause excessive ac losses in the SSD. In addition, the capacitors used to correct the power factor could resonate with the SSD causing excessive voltage and/or current flow. The drive loads which change from one cycle to the next could affect the SSD control strategy. Finally, Carolina Power and Light frequently switches capacitor banks at high speed, which could affect SSD operation. So far, the SSD has provided carryover for approximately 40 events.

\section{Cyanco Liquid Cyanide Plant in Winnemucca. Nevald}

An SSD unit was installed in May, 1993 at Cyanco's liquid cyanide plant located in Winnemucca, Nevada. The unit is being leased by Cyanco and was installed in-line : $:$ ith sci. 1 variable-speed motor controllers. This unit is just beginning actual operation.

\subsubsection{Market Evaluation for SSD}

At present, the SSD is serving a niche market in the United States, and SI is the only company active in the field. SI believes that there is a market for several hundred SSD units worldwide. Several companies have expressed an interest in entering the market. General Atomics, Westinghouse, General Dynamics, and IGC in the United States; Siemens AG and Ansaldo in Europe; and several battery manufactures are watching to see if a large enough market develops to justify their entry. In principle, either batteries or flywheels (with or without magnetic bearings) could compete with small-scale SMES for this market. Should the cost of high-performance capacitors drop significantly, they could also be a competitor. A PCS would be a common component in all of these systems.

Figure 5-4 compares the energy and power density for several proposed storage system: $: 15$ Conventional lead-acid batteries have the lowest specific power density, which determines the

15 This plot excludes the effect of the PCS, which would be common to all systems. 


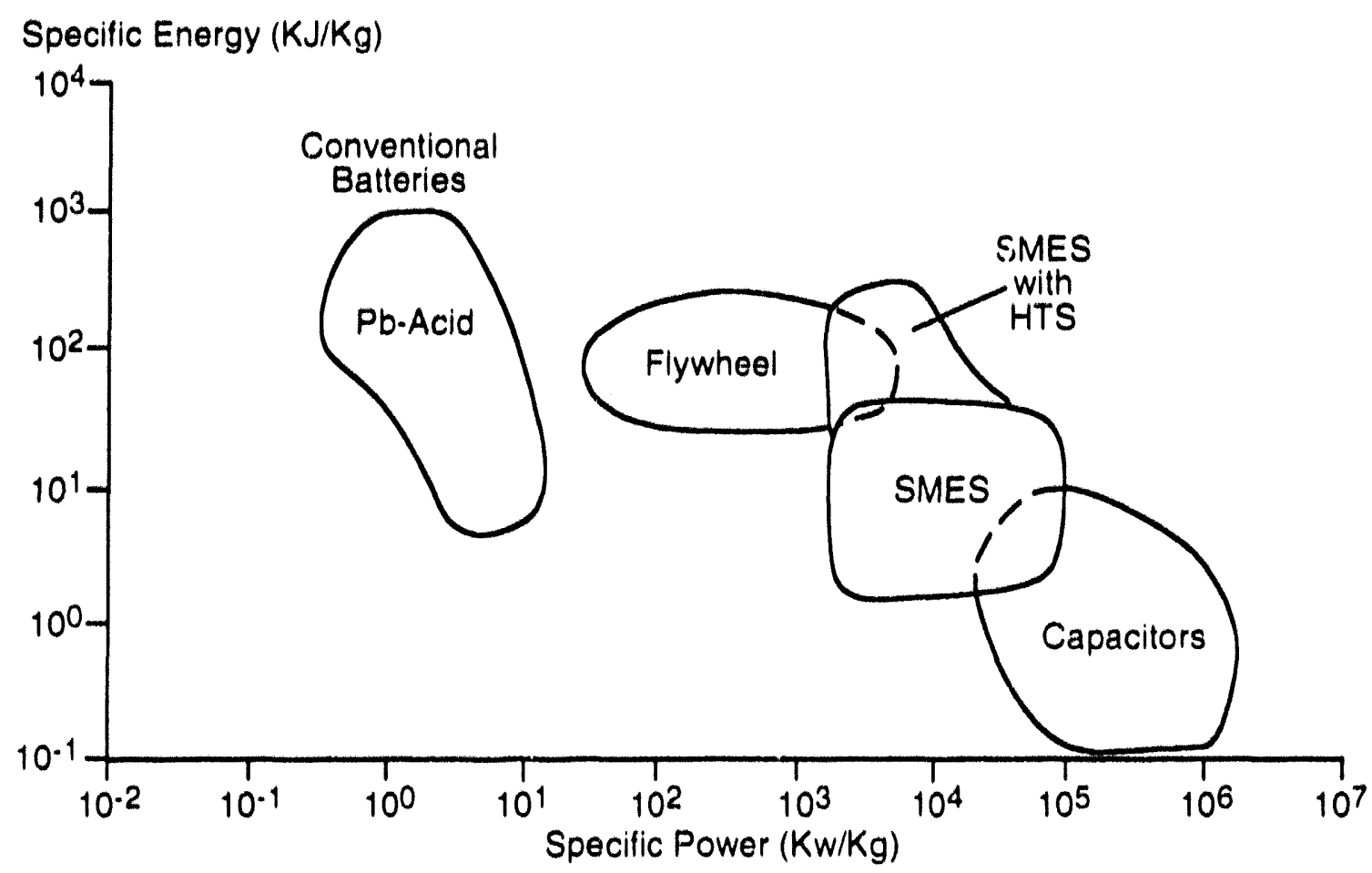

FIGURE 5-4 Comparison of Specific Power and Energy Density for Several Proposed Storage Systems

battery mass required for this application. However, battery manufacturers have expressed confidence that this value could be inceeased significantly, particularly for periods limited to several seconds, were there a large enough potential market. The mass for capacitors and SMES units is determined by their specific energy density. Flywheels have the best match of specific power and energy. The increase in specific power for an HTS SMES is due to the likely greater stability associated with operating at $77 \mathrm{~K}$ which should permit larger ac losses to be tolerated during rapid charge and discharge.

Because no standards have been established, the real problem associated with power quality is whose fault it is and who should pay for it: equipment manufacturer, electric utility, or the customer. This affects how an SSD or similar product is marketed. Present rate structures do not adequately address the power quality issue. A simple example illustrates this: assume that a voltage sag causes a piece of equipment with a power rating of $1 \mathrm{MW}$ to be out of service for eight hours. Assume such an outage occurs 20 times per year. Taking a representative cost of electricity of $\$ 0.15 / \mathrm{kWh}$, the annual cost to the utility in lost revenue is $\$ 24,000$. Assuming that an SSD unit costs $\$ 600,000$, this would result in a simple payback of 25 years, too long for most companies to 
even consider. ${ }^{16}$ In contrast, if we assume that the cost to the company of each outage is $\$ 10,000$, similar to the experience at the Sonoco Bag Plant, with the same 20 outages per year, the cost savings from the customer's perspective is $\$ 200,000$. This pays back the cost of an SSD unit in only three years, making the SSD a very reasonable investment.

Initially, as part of R\&D effort, utilities have leased the SSD unit. More recently, customers have begun leasing SSD units directly from SI. In the future, customers may opt to purchase their own SSD unit or pay the utility a special fee to provide improved power quality. This would allow the utility to buy or lease the SSD and make it available to selected customers. However, in the long run, it might turn out to be more cost-effective for equipment manufacturers to make equipment more tolerant to voltage sags and/or momentary power outages or to provide an internal backup capability.

\subsection{Potential Applicalion of HTS to Small-Scale SMES}

The earliest application of HTS will probably be as a current lead operating between $77 \mathrm{~K}$ and $4.2 \mathrm{~K}$ in an SSD unit. The current leads in the present SSD unit represent a significant part of the refrigeration load. HTS-based leads could greatly reduce both conduction and 12R losses relative to conventional current leads. At present, SI would use the HTS-based current leads to extend the ability of the liquid helium system to carry over in the event of a loss of refrigeration. rather than decrease the refrigeration capacity. A collaborative effort between Argonne National Laboratory and SI is presently underway to develop such a lead.

The present LTS-based SSD consumes approximately $2720 \mathrm{MJ} / \mathrm{d}$, while an HTS-based SSD might only consume $148 \mathrm{MJ} / \mathrm{d}$. As a percentage of storage capacity (1 MJ), both of these numbers are huge compared to a large-scale unit where the daily consumption for refrigeration is only a few percent of the SMES stored energy. However, in addition to electricity consumed for the SSD refrigeration system, a significant amount of electricity is also required for the air conditioner used to cool the trailer housing in the SSD system.

An analysis by W.J. Schafer and Associates $|17|$ indicates that an all-HTS-hased SSD design could be more compact, simpler (it may not even need a refrigeration system), and could greatly reduce power consumed by the refrigeration system. For purposes of their analysis, they assumed that HTS can be manufactured in $\mathrm{km}$ lengths with a $J_{c}$ of at least $20 \mathrm{kA} / \mathrm{cm}^{2}$ when operating in a magnetic field of $4 \mathrm{~T}$. They also assume that the conductor has enough mechanical strength to be twisted so as to reduce ac losses. They estimate that the presently available conductor using the powder-in-silver-tube technique can only achieve the desired current density when operated at $20 \mathrm{~K}$ and would have a cost of $\$ 2,000 / \mathrm{kg}$. Figure $5-5$ shows the results of their analysis and shows that an HTS-based SSD using today's technology would probably cost about $30 \%$ more than the present SSD unit. Figure 5-5 also illustrates that the PCS represents over half the cost of the present SSD unit.

16 To date. SI has not quoted a price for an SSD unit. All SSD units have been leased, not sold. The present ledse price is $\$ 25,00()$ ner month, plus a $\$ 25,0(0)$ pickup and delivery charge. 


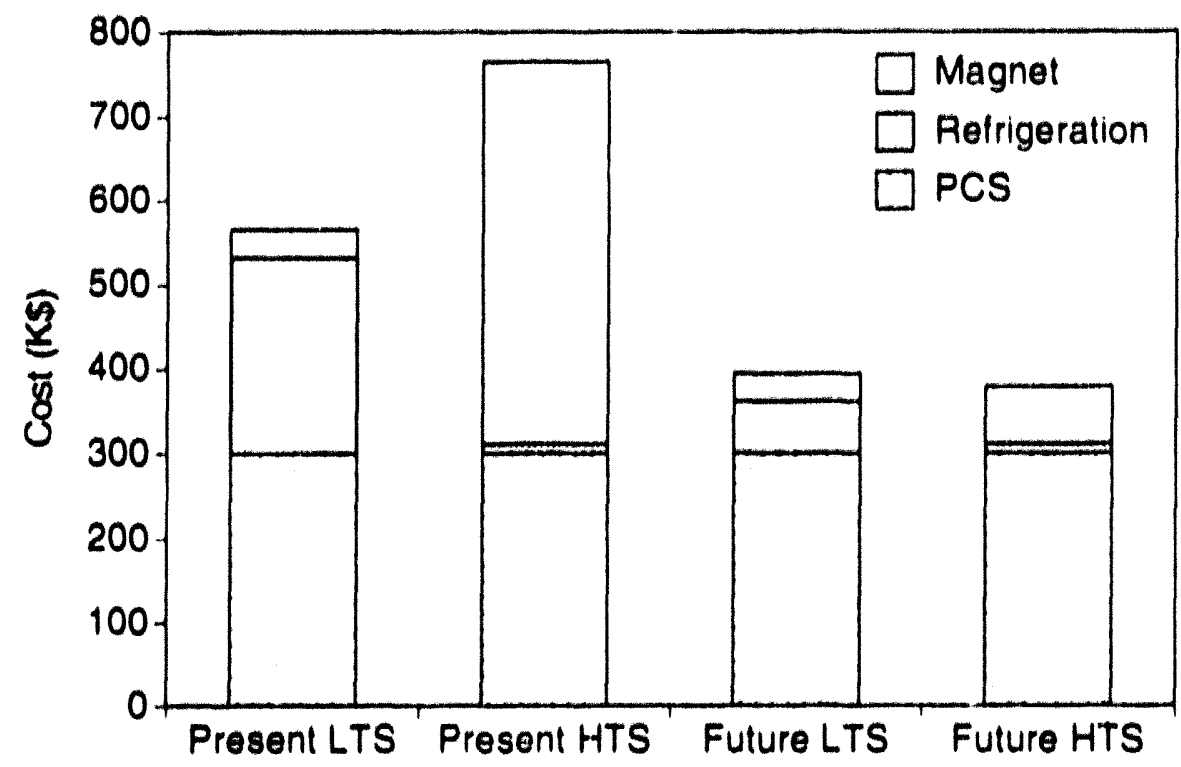

FIGURE 5.5 Comparison of Costs for a Present and Future LTS.Based and HTS-Based SSD Unit

Assuming that the HTS could be improved so as to achieve the desired current density at 77 $K$, and assuming that the cost of HTS conductor could be reduced to $\$ 120 / \mathrm{kg}$ (comparable to $\mathrm{Nb}_{3} \mathrm{Sn}$ conductor), an HTS-based SSD would be approximately $30 \%$ cheaper than the present SSD unit. However, the analysis by W.J. Schafer and Associates showed that by using a more efficient refrigerator and by installing a heat shield, the costs of the present SSD unit could also be reduced by about $30 \%$. The use of an HTS-based current lead would most likely further reduce the cost of the SSD unit. However, the PCS represents approximately $75 \%$ of the total SSD cost for the future HTS- and LTS-based designs. Thus, reducing the cost of the PCS would have the greatest effect on total system cost. 


\section{Medium-Scale SMES}

\subsection{Introduction}

A number of potential applications have been proposed for medium-scale SMES. These include smaller versions of a large-scale SMES for load leveling at a distribution substation or customer-side-of-the-meter :t a large commercial or industriai facility, as well as for spinning reserve and fast-power reserve: At the other extreme are larger versions of a small-scale SMES for providing improved power quality or custom power for larger facilities such as for electric train or transit systems. In addition, several new applications for SMES on transmission systems have emerged. These include transient stability enhancement. power oscillation damping. and voltage regulation. Each of these requires that the PCS be capable of absorbing and generating hoth real and reactive power.

\subsection{SMES on Transmission Systems}

A recent issue of the EPRI Joumal $|18|$ contained an article hypothesizing the transmission network of the future based upon the Flexible AC Transmission System (FACTS) concept in which SMES would play an integral part. FACTS would incorporate power conversion technology throughout, with SMES absorbing and generating both reactive and/or real power. The FACTS system would enable transmission systems to hecome more reliable, more highly automated, self-correcting, and more productive. In addition. FACTS would increase the capacity of existing lines and strengthen ties among power pools. According to the article. a FACTS system could save North American electric utilities billions of dollars by reducing the need for future generation capacity and transmission lines.

The first step toward demonstrating the pussibility of such a system took place in 1984 when a 30 MJ SMES unit was used to stabilize the Pacific Intertie between the Pacific Northwest and Los Angeles (see Section 4.1) |19|. Subsequently, Japan examined using an SMES to improve Japanese transmission lines. More recently, Hydro Quebec has performed extensive modeling of how an SMES might be used on their system.

Hydro Quebec performed a full-scale simulation of how an SMES unit might affect their system [20]. Hydro Quebec has $28 \mathrm{GW}$ of installed hydro capacity operating at remote localtions throughout their service area. These units are tied together with a $735 \mathrm{kV}$ transmission system. Presently, the system has nine synchronc si, condensers totaling 3,150 MVar of capacity, II static var compensators in the 4,400 MVar total dynamic range, 13,545 MVar of series compensation, and a $2,000 \mathrm{MW}$ de line operating at $+.450 \mathrm{kV}$ and spanning a distance of $1.500 \mathrm{~km}$. They choose to model a 1,000 MJ (278 kWh) SMES unit with a 640 MVA PCS. They assumed that the PCS consisted of a four quadrant GTO thyristor based ac-de converter with a capability to absorb and inject up to 640 MW of real power, to absorb and inject up to 640 MVar of reactive power, or 
anything in between. A preliminary analysis indicalled that SMISS would he somewhat more expensive than conventional lechnology, hut would provide superior performance.

The simulation examined (wo possible uses of an SMISS unit: (1) acting as a voltage regulator. and (2) acting as a virtual generator. An SMISS unit can provide vollatge stabilizaltion by absorbing and injecting reactive power along a long transmission line lo improve system vollage and to increase the capability of a given line to tratnsmit power. The analysis showed that an SMIES unit provided betfer stabilization than a conventional static var compensator (SVC), especially during a large disturbance when the line is heavily loaded (i.e., the line vollage is reduced). This is because an SVC is essentially a capacitor. Consequently, the amount of charge stored within the SVC is propontional to voltage. As the line voltage declines during periods of heavy loalding. the ability of the SVC to inject the additional current needed to stabilies the vollage decreases. In contrast, a SMES unit is essentially a de current source and is unallected hy the lime voltatge

When a SMES acts as a virtual generalor it improves the transient stahility and syslem damping on a transmission line. What happens is that during a latge disturbance. vach an a temporary short circuit caused by a lightning strike or two lines touching during a wind storm, lhe force (e.g., steam) driving the generator can cause the generator to acecelerate and become out of phase with the rest of the system. A SMISS unit can be used to ahsorth this excersive killelle energy during the subsequent oscillations and return energy to the system. if necessary, Io improve the system transient stability and to increase damping of system oscillations after the disturbance subsides. Consequently, oscillations of the system are reduced, and the effective transmission capacity of the system is increased. The Hydro Quehes studies showed hat the SMISS unit was most effective when lixialed near the generator most susceptible to the risk of loss of synchronism.

For several years, Hydro Quebec has bect carrying on analyses of possible uses of anl SMES on their system. Beciause of the large number of possible locilions for sitting an SMI:S unit and because of the large number of different lypes of disturhances which cinn oceur on such a large system. further extensive studies are needed of how and where to Use an SMES unit and whal the total henefits might be. Although initial results indicale that an SMES is more expensive hut provides superior performance compared to conventional lechnolongy, it is loo carly fior delinitive conclusions; the analysis continues.

\subsection{SMES for Customer Load Leveling}

Use of a medium-scale SMLS unit lo provide load leveling for a large commercial or industrial customer has been proposed numerous times. One of the problems in justifying ant SMES for such an application, is that the unit cost of a SMLS in dellars per $k$ Wh increases will decreasing stored energy. Although a large-sciale SMLS unit with a calpacity of $5 .(1)(x)$ MWh might prove cost-eflective relative (o) a gas turbine or another storage lechnology, such a unit is unlikely to be cost-effective al smaller sizes under normal eonditions. What is needed is to either identily all application where the SMLS provides exceptional value (e.g., a small-sciale SMISS used (o) improve 
power quality) or where the cost of the SMES unit can be justified for another application (e.g. such as where the magnet is required for magnetic separation)

A Japanese study 1211 illustrates the need to reduce the cost of an medium-scale. L.TS hased SMES Io justify use for customer load leveling. This study eximined an antual 3().story office building. They calculated the patterns of energy use with and wilhout an SMISS unit with a capacily of $6 .+\mathrm{MWh}$ and $1,+\mathrm{MW}$. They used actual lime-of-use ralles for energy and demand costs to calculate the break-even cost of the SMES unit at 1.58 billion yen. Next, they calculated that cost of a solenoidal SMES unit to be three times the allowed break-even cost. and that the cost of a toroidal SMES unit would the six tumes the allowed break-even cost. They also calculated that the round-trip efficiency of such an SMES unit would be anly 75\%, slightly hefler than for a compurable buttery storage system.

\subsection{SMES for Stationary Transportation Applicatlons}

Severul groups have proposed using a medium-scale SMES unit to either load level (or (o) reinforce volfage on an electric train system. A joint collaboration teitween the Paul Sicherrer Institute (PSI) and the Swiss branch of ABB proposed building a 250) $\mathrm{kWh} / 20 \mathrm{MW}$ demonstration SMES unit for use on the Swiss electric train network. The unit would provide energy fo assist trains in accelerating out of a station and absorb energy generated by trains deceleratting into a statton. However, this project has not proceeded due to a lack of lunding.

As mentioned in Section 5.2. P(idl: issued a request for proposals to examine means for solving a volfage sag problem on the BART system. The functional specification was issued on December 11, 1992 calling for proposed systems that are capable of delivering $8 \mathrm{MJ}^{17}$ of energy at a voltage of $8(x) \mathrm{V}$ de (this implies a current capability of $4,()()() \mathrm{A}$ ). SI. Westinghousc, and General Dynamics responded with propessals incorporating a medium-scale SiMES unit. Bechtel submitted a design hased upon the use of standard-starting. lighting. and ignition (St.I) balleries used in conventional automobiles. There was also a proposal to use conventional pulsed-duty rectifiers.

According to Melissa Reading. PCi\&le project manager, the battery proposal had the lowest cost. However, because of the limited ability of batteries to provide high levels of power, this option turned out to be bulky and may not be aceeptable for use in tunnels where space is at a premium. In addition, the limited lifetime associaled with ballery systems would require replacement of the batteries after several years. BART expressed some concern ahoul coming up with additional capital equipment money to repli ot the batteries at a future date. The pulse rectitier option had a cost slightly less than the SMES options, hut it would require the installation of additional cable capacity along the line. Surprisingly, the eost for the three SMISS proposals differed by almost a fiactor of three. A linal report is expected to be out during fiall 199.3.

17 This is stlll a very small SMLSS test unll A larger, medium-scale unil minght be needed fur the entire BNRT system. 
Early in 1994. PGikE would like to begin testing. first at the Hayward test facility and then on an above-ground section of BART. Although a final decision has yet to be made, the SMES option is favored at present. Because the Westinghouse and Cieneral Dynamics units are not commercially available, the SI unit would the the likely chosice.

\subsection{Potential Impacts of HTS-Based SMES Systems}

An HTS-based SMES could have lower cost and increased efficiency compared with an L.TS-based system. As shown in Figure 3.1, refrigeration and helium-related cosfs account for over 50\% of capital cost for a 10 MWh SMES unit. In addition, the refrigeration unit consumes over $15 \%$ of the energy stored in a I(X) MWh SMI:S unit each day. Consumption is even larger for smaller units. An HTS-based SMES operating at $77 \mathrm{~K}$, ralher than $1.8 \mathrm{~K}$. could eliminate the majority of refrigeration and helium-related capital costs and reduce refrigeration consumption up to a fuctor of almost $6(0)$. In addition, operation at higher temperutures would permit the use of cryocoolers which could greally improve reliability and reduce muintenance costs.

Another option would be to operate an HTS-based SMES at approximately $20 \mathrm{~K}$ and take advantage of the ability of hismulh-based material to operate at high-magnetic fields to build an SMES that operates at $20 \mathrm{~T}$ instead of $5 \mathrm{~T}$.

As shown in Tuble 6.1, operating at a higher magnetic lield could result in a more connpact design and reduced conductor requirements |22|. However, the larger magnetic forces would require more cold mass structure and the brittle nature of HTS material would require careful design to muke sure that the conductor is not subjected to excessive stress.

TABLE 6.1 Comparison of Radius, Surtace

Area, and Conductor Requirements lor an

SMES Operating at $5 \mathrm{~T}$ and $20 \mathrm{~T}$

\begin{tabular}{|c|c|c|}
\hline \multirow[b]{2}{*}{ Parameler } & \multicolumn{2}{|c|}{ Operating Magnelic Fiold } \\
\hline & $5 T$ & $20 \mathrm{~T}$ \\
\hline $\begin{array}{c}A(m) \\
\text { is }(A m) \\
A\left(m^{2}\right)\end{array}$ & $\begin{aligned} & 20.8 \\
& 8.3 \quad 10^{8} \\
& 238.7\end{aligned}$ & $\begin{array}{l}8.25 \\
5.23 \quad 10^{8} \\
37.6\end{array}$ \\
\hline
\end{tabular}


Recently, W.J. Schafer Associates, Inc, and Cieneral Dynamics, with funding from the U.S. Department of Energy, performed a preliminary design study for an HTS-based SMES 231 . They considered medium-scaled SMES units with a capacity ranging from 2 to 20() MWh 17.2 10 720 (iJ) and a power rating of $4.4(0) \mathrm{MW}$. To reduce fringe fields, only toroidal designs were considered. An operating magnetic tield of 10 t' was chosen to take advantage of the ability of bismuth-strontium-calcium-oxide superconductor to operate al high-magnetic ficld all $20 \mathrm{~K}$. They adopted a modular design that maintained the HTS material under compression (see Figure 6-1) A conductor current of $10 \mathrm{kA}$ was selected 10 reduce conductor length and to reduce the peak voltage across the coil.

Because of the higher operating magnetic field. the coil dimensions for the HTS design were approximately a factor of two smaller than for the comparable LTS design. In addition. Ihe HTS unit had a reduced refrigeration requirement. Operating at $20 \mathrm{~K}$ reduced the refrigeration requirements by about $60 \%$ relative to an LTS design operating at $4.2 \mathrm{~K}$. Were it possible 10 operate the HTS SMES at $77 \mathrm{~K}$, refrigeration requirements would be reduced by approximately 90\%. However, use of HTS current leads could reduce the refrigeraltion load in an LTS SMI:S by approximately $25 \%$ for a 2 MWh unit.

Assuming that the HTS conductor had a $J_{c}$ of $20.0(0) \mathrm{A} / \mathrm{cm}^{2}$ at $20 \mathrm{~K}$ in a magnetic lield of 10 $\mathrm{T}$ and a conductor cout of $\$ 10(0) / \mathrm{kg}$ (equal to that of NbTi conductor), the study found that the cost of an HTS bused SMES would be approximately a factor of two to three more expensive than the comparable LTS-based SMES. ${ }^{\text {in }}$ Since the cost of the conductor became dominant at smaller

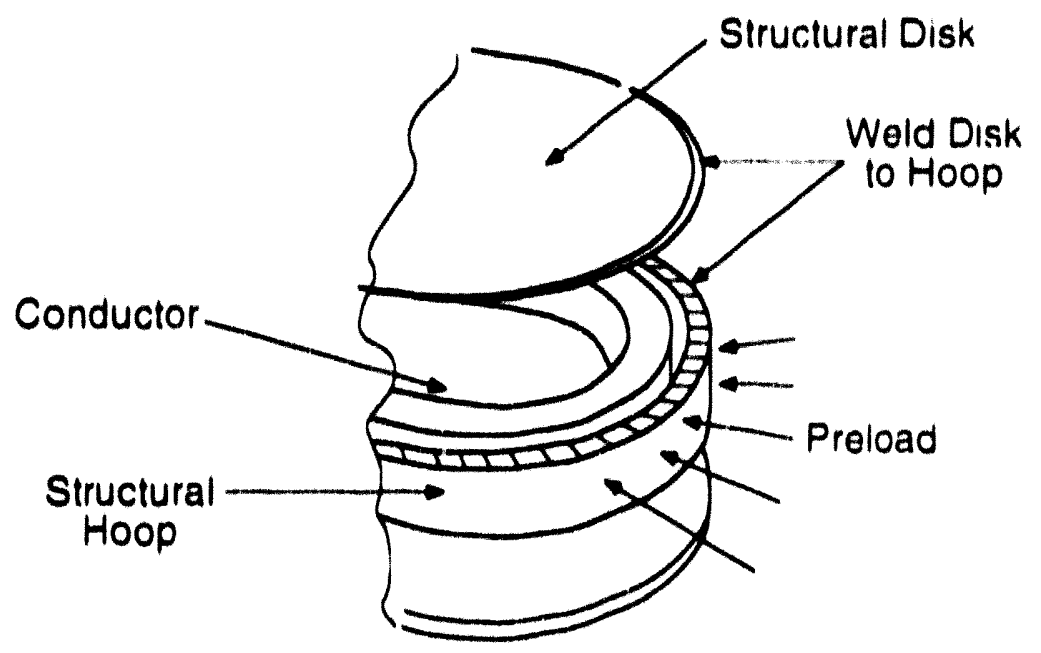

FIGURE 6.1 Illustration of Design for an HTS.Based SMES Module by W.J. Schater Associates, Inc.

is This is due to the lower Je values and lower mechantical strength associaled will presemt day powder-ill-bilver. tube BSCC(O) wire and tape. 
SMES sizes - the cost of the conductor amounted to 8()$\%$ of total cost for a 2 MWh unit and $50 \%$ of the total cost for a 200 MWh unit - the only practical way to reduce the cost of an SMES unit would be to reduce the cost of the conductor. As shown in Equation 3.4, the only way to reduce the cost of the conductor is 10 achieve a higher de value or to reduce the HTS dollar per kilogram cost below that for NhTi. The analysis also concluded that increasing the strain tolerance of HTS conductor would reduce the amount of cold structure required, thereby reducing the cost somewhat. 


\section{Flywheel Storage with Superconducting Magnetic Bearings}

\subsection{Introduction}

Flywheels store energy in the form of rotational kinetic energy. If a flywheel is connected to a variable-speed, motor/generator unit through some form of PCS, electrical energy can be converted to kinetic energy, stored in a flywheel, and converted back to electrical energy. An early application of this principle, first used in the 1960s, was to smooth out cyclical power requirements for synchrotron accelerators. Argonne National Laboratory installed a $400 \mathrm{MJ}$ flywheel system on the Zero Gradient Synchrotron (ZGS) Accelerator to smooth out power pulses. The ZGS ramped up on a 4-s cycle. The flywheel was capable of delivering $20 \mathrm{MVA}$ to the ZGS per cycle and atsorbed $12 \mathrm{MVA}$ during recharge. The flywheel was a solid, laminated steel structure approximately $60 \mathrm{~cm}(2 \mathrm{ft})$ high by $300 \mathrm{~cm}(10 \mathrm{ft})$ in diameter and operated at a maximum speed of $800 \mathrm{rpm}$, corresponding to a tip speed of approximately $230 \mathrm{~m} / \mathrm{s}(750 \mathrm{f} / \mathrm{s})$. Windage and bearing losses amounted to approximately $1 \mathrm{MW}$. Similar devices were installed at accelerators at Brookhaven National Laboratory and Lawrence Berkeley Laboratory.

The energy stored in a flywheel is equal to $\mathrm{J} \omega^{2} / 2$ where $\mathrm{J}$ is the moment of inertia of the flywheel, and $\omega$ is its angular velocity. For a given flywheel mass and geometry, the amount of energy that can be stored in a flywheel increases quadratically with increasing $\omega$. However, as $\omega$ increases, the centripetal force also increases until it exceeds the molecular forces holding the flywheel together, causing the flywheel to shatter. The centripetal forces increase with increasing radius. Thus, the largest force occurs at the outer edge of the flywheel. The corresponding hoop stress is proportional to the square of velocity of the flywheel at the tip, sometimes referred to as tip speed, and the density of the material. Consequently, the maximum allowed tip speed determines the maximum value for $\omega$. For a given $\omega$ and flywheel mass, stored energy is maximized for a structure having the largest value of $\mathrm{J}$. The simplest form of a flywheel is a solid, rotating, steel cylinder. However, a flywheel in the form of a bicycle wheel with most of the mass concentrated at the rim has a $\mathrm{J}$ almost twice as large. The goal in flywheel design is to concentrate most of the flywheel mass at the rim while maintaining structural integrity.

During the late 1970s to mid 1980s, much effort was devoted to developing compact, efficient flywheels with relatively high specific energy for use in vehicles. Various composite materials, flywheel shapes, and motor/generator configurations were developed and tested. By 1985, a number of flywheels had been built and tested. Composites such as carbon/epoxy and Kevlar produced flywheels with the greatest specific energy, some exceeding $200 \mathrm{Wh} / \mathrm{kg}$ for the rim portion of the flywheel. These flywheels had tip speeds in excess of $1,000 \mathrm{~m} / \mathrm{s}$, three times the speed of sound. One flywheel failed at a tip speed of $1,405 \mathrm{~m} / \mathrm{s}$. At these speeds, the flywheel must operate in a vacuum to prevent thermal burn up. To keep losses due to molecular drag low, a vacuum of approximately $0.1-0.01 \mathrm{~Pa}\left(10^{-4}-10^{-5} \mathrm{Torr}\right)$ is required. 
Figure 7-1 illustrates one such system that was assembled at Oak Ridge National Laboratory. The various components of this system were tested independently; the complete system was never tested because of funding constraints. The carbon/epoxy composite flywheel was designed to operate at a tip speed of $1,200 \mathrm{~m} / \mathrm{s}(33,000 \mathrm{rpm})$, to weigh $12.7 \mathrm{~kg}(28 \mathrm{lb})$, and to store a total of $8 \mathrm{MJ}(2.2 \mathrm{kWh})$. The flywheel was coupled to a motor/generator containing permanent Samarium-Cobalt $(\mathrm{SmCo})$ magnets. The motor/generator weighed $8.2 \mathrm{~kg}(18 \mathrm{lb})$, had a constant power output of $50 \mathrm{~kW}$, and an efficiency of $95 \%$.

In principle, such a system could be scaled up to provide a transportable storage system with a capacity ranging from several $\mathrm{kWh}$ up to $10 \mathrm{MWh}$. However, a potential disadvantage of such a flywheel system is bearing loss. Bearing loss is a constant loss independent of how rapidly the device is cycled, much like the refrigeration load in an SMES system. This is not a problem for hybrid-vehicle applications where the flywheel is cycled rapidly (a typical cycle lasts several minutes). However, for diurnal load leveling, or an all-flywheel vehicle, round-trip efficiency would be too low to even consider. For example, if a permanent magnet were used to provide axial support and a mechanical bearing were used to provide radial support, bearing loss over a daily cycle would amount to approximately $48 \%$ of the energy initially stored in the flywheel.

One way to improve the system would be to use an electromagnet to provide axial support and a passive magnet with electronic feedback for radial support. A conventional bearing would be used to provide emergency support should the flywheel touch down. Proposed designs for flywheel vehicle applications indicate that such techniques might reduce bearing losses by up to a factor of 10 or more when the vehicle is parked. Losses would be higher during actual driving because of additional forces imposed on the flywheel due to road conditions. The use of HTS bearings might reduce stationary losses even further as well as eliminate the need for the electronic feedback circuit. However, the Superconducting Magnetic Bearing (SMB) would require a refrigeration system.

\subsection{HTS Magnetic Bearings}

Using conventional magnetic bearings to reduce frictional losses poses a severe limitation. In 1842, Earnshaw [24] proved that a system consisting only of magnets and paramagnetic materials is unstable. Consequently, the passive magnet used to provide radial support in a conventional flywheel requires sensors and an electronic feedback system to constantly correct the inherent instabilities present in such a system. In addition to adding to the complexity of the device, the electronics consumes energy contributing to inefficiency. However, HTS can be introduced into such a system to provide stability without the need for electronic feedback.

Ore of the most common pictures associated with high-temperature superconductivity is the demonstration of a permanent inagnet being stably levitated above a piece of HTS, usually yttriumbarium-copper-rxide (YBCO) immersed in liquid nitrogen [25]. The fact that the magnet stably 


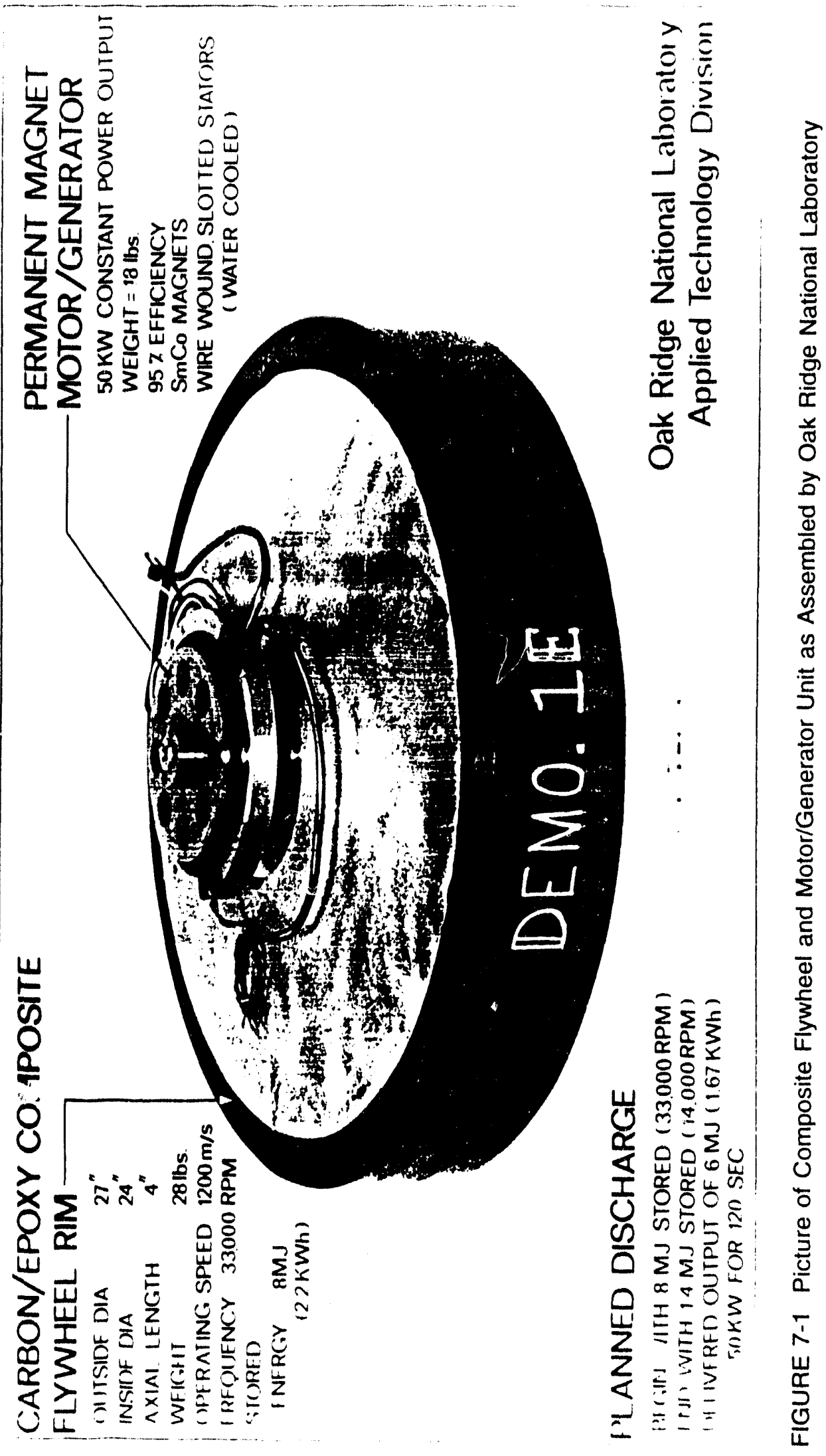


rotates above the YBCO appears to violate Earnshaw's theorem. However, the YBCO acts as a diamagnet with hysteresis and is thus not subject to Earnshaw's theorem [26]. When a permanent magnet is moved across a piece of YBCO, the magnetic hystersis behavior of YBCO causes a drag to oppose the motion. However, this drag only occurs if the motion of the magnet causes a change in the magnetic field in the volume occupied by the HTS. Consequently, this drag can be made negligibly small in an SMB by ensuring rotational symmetry of the magnetic field $|27|$. This behavior makes the building of highly efficient SMBs possible.

Soon after the discovery of HTS material, several researchers built small devices incorporating SMBs. F. Moon and P.-Z. Chang at Cornell University [28] built a device with a small rotor suspended by an SMB that achieved a rotational speed of $120,000 \mathrm{rpm}$. About the same time, R. Takahata and T. Yotsuya at Koyo Seiko Co. Lid. [29] built and tested a similar device at speeds up to $10,000 \mathrm{rpm}$. However the performance of these devices was hampered by two factors: (1) low levitation force, and (2) low stiffness. The levitation force in a simple SBM provides no more that $0.01 \mathrm{MPa}$ (1.5 PSI) of lift relative to the cross section of the magnetic rotor $[30]$.

The levitation and stiffness of an SBM depends upon its $J_{\mathrm{c}}$ flowing within single grains of material, unlike HTS wire where transport current must flow from one grain to another. This makes it easier to produce suitable bulk material with high $J_{c}$ values. Present SMBs are made from high-quality, melt-textured YBCO. Consequently, further improvements in $J_{c}$ will likely be difficult. However, it is possible to provide additional lift through the use of permanent magnets or electromagnets.

The ability of an SMB to prevent lateral displacements of the rotor depends upon its stiffness. A recent measurement at the Texas Center for Superconductivity at the University of Houston ( $T_{C} S U H$ ) has obtained a stiffness of $1-3 \mathrm{~N} / \mathrm{mm}$ for lateral displacements and up 10 $12 \mathrm{~N} / \mathrm{mm}$ for axial displacements in their SMB device. In the case of stationary flywheel applications, the load is expected to be essentially steady. Consequently, mechanical bearings could be installed to prevent catastrophic bchavior should the device fail, while the SMB would provide stabilization under normal operating conditions.

Using an electromagnet to provide lift and an SMB to provide stabilization, $T_{C}$ SUH has measured lifting capacities in excess of $0.41 \mathrm{MPa}(60 \mathrm{psi})$ [31]. This value should be more than enough to permit building a modular flywheel for various utility applications. Recently, Koyo Seiko, using a similar technique, levitated a $2.5 \mathrm{~kg}$ motor/shaft and spun it at $42,(0)() \mathrm{rpm}$ using improved YBCO material [32]. Figure 7-2 illustrates Koyo Seiko's device.

\subsection{Flywheel Storage System}

A modular, flywheel storage system for electric utility applications offers several advantages. First, the units could be assembled at the factory and delivered to the site by truck for 


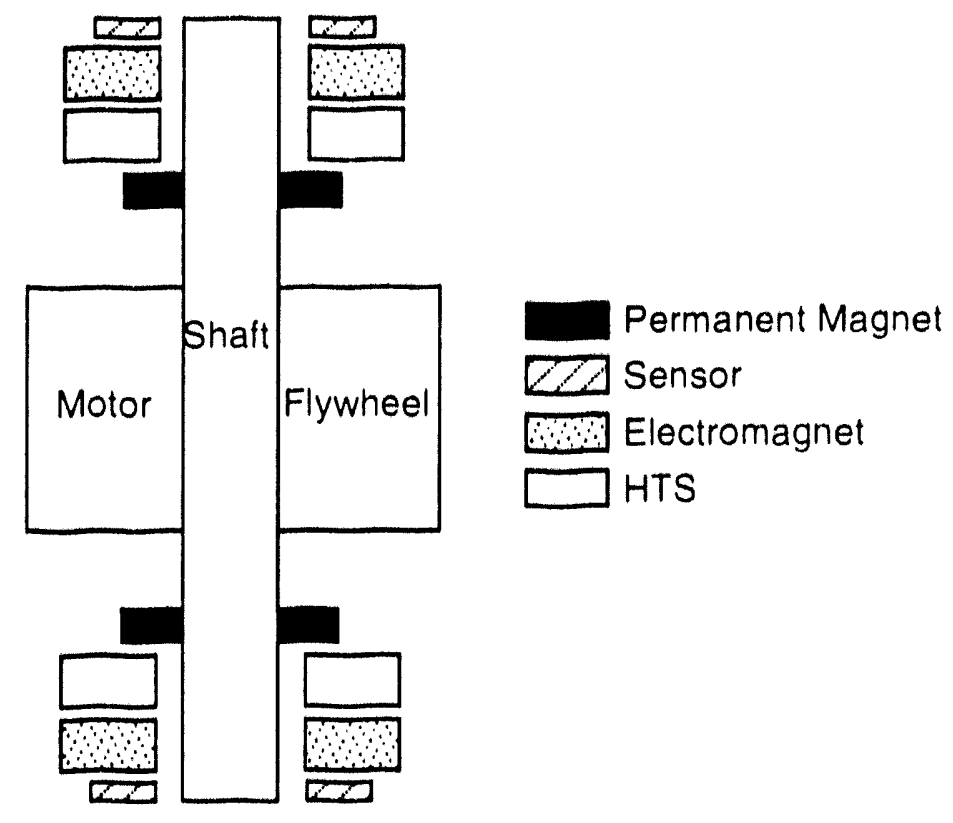

FIGURE 7-2 Illustration of Magnetic Bearing Built by Koyo Seiko Company in Japan

final installation. This would reduce on-site construction costs. Second, flywheel balancing, critical to flywheel performance, could be performed under controlled conditions by using sophisticated equipment. The concept is to build a module that would fit on a tractor trailer truck. In the United States, this limits the total size of the device to an approximate diameter of $275 \mathrm{~cm}$ $(9 \mathrm{ft}$ ) and a weight of approximately $30 \mathrm{t}$. A specially designed tractor trailer might extend these limits somewhat.

Oak Ridge National Laboratory examined what a module might look like. A module would consists of a flywheel that is $2 \mathrm{~m}$ in diameter by $2 \mathrm{~m}$ tall that rotates about the vertical axis and a motor/generator set. Each flywheel assembly would be housed in a sealed vacuum system with a connection to an external pumping system. The flywheel would be supported by combination of permanent and electromagnets with electronic feedback control.

The Oak Ridge design for the flywheel $r$ ii ploys a fiber with a maximum working stress of 4,800 MPa (700 ksi) and a corresponding laminate maximum working stress of 3,400) MPa $(500 \mathrm{ksi}){ }^{19}$ The density of the laminate is $1,600 \mathrm{~kg} / \mathrm{m}^{3}\left(0.058 \mathrm{lb} / \mathrm{i}^{3}\right)$, resulting in a maximum allowed tip speed of $1,465 \mathrm{~m} / \mathrm{s}$. Operating the flywheel at $1,200 \mathrm{~m} / \mathrm{s}$ translates to a working stress

19 The best available fibers have a maximum working stress of approximately 6,90) MPa (1,00) ksi). However, these fibers are much more expensive and probably would not be cost-effective in a stationary application where the specific energy of the device is of less importance than in vehicle applications. 
equal to $67 \%$ of the maximum working stress, assuming that the inner radius of the rim is $0.6 \mathrm{~m}$, the flywheel rim mass is $6.400 \mathrm{~kg}$, and the energy stored in the rim is $880 \mathrm{kWh}$.

To effectively use the energy stored in the flywheel, the motor/generator set must be capable of working efficiently over a large speed variation. Since the energy stored in the llywheel is proportional to $\omega^{2}$, a speed variation of a factor of two will allow recovery of $75 \%$ of the energy stored in the flywheel, while a factor of three would recover 9()$\%$, but might require use of a more complicated and expensive motor/generator set. With a flywheel speed variation limited to a factor of two, the usable energy stored in the rim is $660 \mathrm{kWh}$. In addition, a small amount of energy will be stored in the remainder of the flywheel assembly and in the motor/generator set. If we assume that the balance of the system doubles the total mass, then one truck can easily carry two flywheel modules with a usable energy capacity of approximately 1.4 MWh.

An important consideration for a diurnal storage device is its efficiency. Three factors affect overall efficiency: (1) conversion losses in the motor/generator system, (2) losses in the PCS, and (3) losses within the flywheel. Within the flywheel system, there are three sources of loss: (1) windage drag loss, (2) flywheel bearing loss, and (3) motor/generator bearing loss. These three are very similar to the refrigeration power consumption in an SMES system in that they occur whether or not the flywheel system in being actively used. A diurnal flywheel system would most likely employ a combination of permanent magnetic and active magnetic bearings. Recent advances in design promise to greatly reduce bearing losses in stationary hywheels. A magnetic bearing developed by AVCON in California uses homopolar permanent magnets to provide primary bearing suppont with a lift force of up to $2 \mathrm{MPa}$ (285 psi) relative to the air gap |33|. A set of small electromagnets is used to compensate for instahility. Such a system holds promise for very low bearing loss while providing large stiffness, up to $85,000 \mathrm{~N} / \mathrm{mm}(500,000 \mathrm{lb} / \mathrm{in})$. According to calculations by Pat McMullely at AVCON, such a system should have a diurnal loss of approximately $2.4 \%$ per day $[34]$ (this includes all the energy required to run both the electromagnet and the associated electronics). ${ }^{20}$ AVCON is setting up experiments to validate these numbers under laboratory conditions.

The advantage of such a storage system to the utility would be the relatively high specific energy of the system, a long shelf life, no degradation with repeated cycling, high round-trip efficiency, potential for fast discharge, and environmental acceptability. These are many of the same benefits that an SMES unit would have. However, because the flywheel units are modular. the units could easily be moved from site to site as needed and the economics of the system would be unaffected by the amount of energy stored. Thus, if a module was cost-effective for diurnal load leveling, the storage system could be almost any size. In contrast. SMES units are cost effective for diurnal load leveling only in very large sizes $(5,0)(0) \mathrm{MWh}$ or more). To date, no cost

20) This number refers to stationary applications only and is much lower than previous values reported in the literature. This number would be much higher under conditions experienced in a hybrid or all-flywheel vehicles because the magnitude of destabilising forces during driving would be much higher. requiring much higger electromagnet systems to compensate. This system uses approximately $2 \%$ of the energy required by previous all-electromagnetic designs. 
analyses for stationary flywheels have been published and much work remains to be done to validate such a concept.

\subsection{Argonne/Commonwealth Edison Flywheel Project}

Recently, Argonne has teamed with the Commonwealth Research Corporation, part of Commonwealth Edison Company, to perform research on losses associated with SMBs. Their goal is to achieve an overall flywheel efficiency of $90 \%$ for diumal operation. Assuming a roundtrip efficiency for both the PCS and the motor/generator of $96 \%$ each, bearing losses can be at most $2.4 \% / \mathrm{d}$ or $0.1 \% / \mathrm{h} .21$ Efforts to date have been concerned with measuring bearing losses in an SMB. Several SMBs have been built and spin-down measurements made.

The coefficient of friction $\mu$ is a measure of bearing losses and is defined below:

$$
\begin{aligned}
F_{L} & =M g \\
F_{D} R_{D} & =J \alpha \\
\alpha & =2 \pi d f / d t \\
J & =M R_{\gamma}^{2} \\
\mu & =\frac{F_{D}}{F_{L}} \\
& =\frac{2 \pi R_{\gamma}^{2} d f / d t}{g R_{D}}
\end{aligned}
$$

where:

$$
\begin{aligned}
F_{L} & =\operatorname{lift} \text { force }(N), \\
F_{D} & =\operatorname{drag} \text { force }(N), \\
M & =\text { supported mass }(\mathrm{kg}), \\
g & =\text { acceleration of gravity }\left(\mathrm{m} / \mathrm{s}^{2}\right),
\end{aligned}
$$

21 An implicit assumption is that molecular drag losses can be made small with a sufficiently good vacuum system. 


$$
\begin{aligned}
R_{D} & =\text { drag radius }(\mathrm{m}), \\
J & =\text { moment of inertia }\left(\mathrm{kg} \mathrm{m}^{2}\right), \\
\alpha & =\text { angular acceleration }\left(\mathrm{s}^{-2}\right) \\
R_{\gamma} & =\text { radius of gyration }(\mathrm{m}) \\
\mathrm{f} & =\text { frequency }\left(\mathrm{s}^{-1)},\right. \text { and } \\
t & =\text { time }(\mathrm{s}) \text {. }
\end{aligned}
$$

The percent energy loss per second in a flywheel is given by:

$$
\begin{aligned}
\frac{d E / d t}{E} & =\frac{d\left[1 / 2 J \omega^{2}\right] / d t}{E} \\
& =\frac{2 d f / d t}{f} \\
& =\frac{2 \mu g}{2 \pi f R_{\gamma}} \frac{R_{D}}{R_{\gamma}}
\end{aligned}
$$

If we assume all the flywheel mass is in the rim, then $R_{D}=R_{\gamma}$ and $2 \pi f R_{\gamma}$ equals the tip speed $v$. Assuming an allowable energy loss of $0.1 \%$ per hour and a flywheel tip speed of $1.000 \mathrm{~m} / \mathrm{s}$ the required $\mu$ value is:

$$
\begin{aligned}
& \mu=\frac{d E l d t}{E} v \\
& 2 \mathrm{~g} \\
&=\frac{0.001(\mathrm{~h}) 1,000(\mathrm{~m} / \mathrm{s})}{29.8\left(\mathrm{~m} / \mathrm{s}^{2}\right) 3,600(\mathrm{~s} / \mathrm{h})} \\
&=1.4 \times 10^{-5}
\end{aligned}
$$

If we assume that the SMB loss is deposited in the cryogen and if we assume it takes $14 \mathrm{~W}$ of refrigeration to remove each $W$ of heat deposited at $77 \mathrm{~K}$ to room temperature, then the required $\mu$ is $1.0 \times 10^{-6}$. Argonne has built and tested several SMBs. Early results using a $1.10 \mathrm{gm} \mathrm{YBCO}$ samples yielded a $\mu$ value of approximately $3 \times 10^{-6}$. Recent results using bigger and better YBCO samples has yielded a $\mu$ value of approximately $9 \times 10^{-7}$, which would achieve the desired bearing loss. 
Present efforts are geared toward showing that the low coefficients of friction obtained on small samples at relatively low speeds $(<100) \mathrm{m} / \mathrm{s}$ ) and a single permanent magnet less than $10 \mathrm{~cm}$ (4 in.) in diameter can be extended to larger samples and composite permanent magnets $20 \mathrm{~cm}$ $(8 \mathrm{in.})$ in diameter operating at $1,000 \mathrm{~m} / \mathrm{s}$. Taking the example presented in Section 7.3 and a lift force of $0.4 \mathrm{MPa}$, as achieved by T.SUH, a magnet area equal $1010 \%$ of the flywheel area $(60 \mathrm{~cm}$ in diameter) would be required. For lower lift force values, multiple supports could be used. A simple calculation using the Bean Critical State Model shows that the energy loss $\triangle E$ in the SMB due to drag is given by:

$$
\Delta E=K(\Delta B)^{3} \Delta A / J_{c}
$$

where:

$$
\begin{aligned}
\Delta A & =\text { the area }\left(\mathrm{m}^{2}\right) \\
K & =\text { a geometrical constant. } \\
\Delta B & =\text { magnetic field inhomogeneity }(T / m), \text { and } \\
J_{c} & =\text { critical current density }\left(\mathrm{A} / \mathrm{m}^{2}\right) .
\end{aligned}
$$

Thus, the key to obtaining small losses is to obtain a large lift value permitting the use of a small $\Delta A$, a large $J_{c}$, and a small $\Delta B$. The challenge will be to achieve very small $\Delta B$ in a larger, composite magnets.

In parallel with this project, the team is attempting to gain funding for construction of a fullscale module. This effort would involve Argonne, the Commonwealth Research Corporation. Intermagnetics General (HTS supplier), $T_{\mathfrak{c}} \mathrm{SUH}$ (SMB research), and Allied Signal (flywheel manufacturer). To date, no cost analyses have been published.

The SMB would eliminate the need for an electronic feedback system and the associated power loss associated with a conventional magnetic bearing system. This could reduce the complexity of the system and possibly reduce bearing losses. However, the SMB system would require a liquid nitrogen refrigeration system and its associated power requirements. The goal of both the conventional magnetic bearing with electronic feedback and the SMB is to achieve a similar level of efficiency. Which system, if any, wins, will have to be determined on the basis of cost-effectiveness, as will the viability of the complete flywheel system. Such a system holds promise for use in many of the applications proposed for SMES. The primary advantage would be for smaller sizes where the cost of an SMES becomes quite expensive. 


\subsection{European Community Flywheel Project}

KFK/INPP in Karlsruhe and CNRS in Grenoble are involved in a research project sponsored by the Commission on the European Communtity to develop an SMB for applicition in nywhed energy storage. The project involves eight industrial companies. A number of genmetries involving permanent and SMBs of various shapes have heen examined to optimize bearing stiffness in three dimensions. A prototype bearing has been built and integrated into a $2.9 \mathrm{~kg}(6.4 \mathrm{lb})$ thywheel $335 \mid$. A maximum levitation force of $6.5 \mathrm{~N}$ (2ero gap) was measured. The stored energy at $15.000 \mathrm{rpm}$ was $4.8 \mathrm{Wh}$ and the maximum power was $1.5 \mathrm{~kW}$. The goal is to develop a $22.7 \mathrm{~kg}(50 \mathrm{lb})$ flywheel rotating at $50,000 \mathrm{rpm}$ with a storage capacity of $200 \mathrm{Wh}$ capable of delivering $5 \mathrm{~kW}$ of power. 


\section{Summary and Conclusions}

\subsection{Superconducting Magnatic Energy Storage}

The discovery in the early 1960s of the inter-metallic superconductors, such as NhTi. made possible construction of large superconducting magnets, such as the 12 -ft bubble chamber at Argonne National Laboratory. Design and construction of such magnets demonstrated that they would be able to store large amounts of electrical energy.

The present-day SMES concept was horn in 1971 when Professors Boom and Peterson alt the University of Wisconsin proposed using a multiphase (iractz bridge to provide an interface between a superconducting magnet and the electric utilit.' grid. Such a system was proposed for utility load leveling where it would have a number of advantages relative to other existing and proposed technologies:

- The ability to respond 10 changing loads rapidly, within one or two cycles.

- The potential for a round-Irip efficiency greater than $y() \%$.

- No performance degradation upon repeated cycling (long life)

- Little, if any, adverse environmental impact.

Early analyses showed that the use of conventional, iron/sted structure to comlatin the large magnetic forces in an SMES would be uneconomical and led to the proposal of using struts io Iransfer the force to free surrounding rock or soil. Even with the use of this so-culled warm structure, economics only justified construction of very large units. 5.0(0) MWh or larger. In 1985, a cost analysis indicated that a $5,(0)(X)$ MWh unit would have a capital cost of approximatcly I billion 1985 U.S. dollars. Cost analyses performed in 1992 in support of the Energy Test Module (ETM) project to construct a 20 MWh SMES decisonstration unit indicated that the cost of a $5,000) \mathrm{MWh}$ unit may be higher. This would drive the SMCS break-even size even larger.

There is much utility interest in a large-scale SMFS, and demonstrattion projects are in various states of planning in Europe, Japan. and the United States. The ETM project in the United States is farthest along. Designs for the ETM have been worked out on naper and various components have been built and tested. However, the ending of the cold wast has eliminated the military-related mission for the ETM that threatens to jeopardize receiving authorizalton lo begin construction.

Several sinall test units have heen built employing cold structure. The largest was the $30 \mathrm{MJ}(8.3 \mathrm{kWh})$ unit installed in 198.3 to demonstrate stabilization on a large transmission line 
operating between the Pacilic Northwest and Los Angeles. The unit was a technical success, hut was retired due to budget considerations and the existence of a parallel de line which provided similar benefits.

At present, the only commercially available SMI:S unit is a I MJ/I MW SMI:S unit huilt by SI to improve power quality by preventing voltage sags and momentary loss of power. Instead of having hours of energy capacity as in a load-leveling application. the Sl unit has al most several seconds of energy sapacity. Consequently, even though the unit cost for energy stornge is quite large (approximately $\$ 1,(0)(0 / \mathrm{k} W / 1)$, energy storage aceounts for less than half of the unit's cost: the remainder is related to the cost of the PCS. Furthermore, the value of an SMISS unit for power quality improvement can be much grealer than for losd leveling in certailn industrics. juslifying the high cost.

Allempts to justify medium-siaed SMIS:S units for load leveling have lailed hecause the

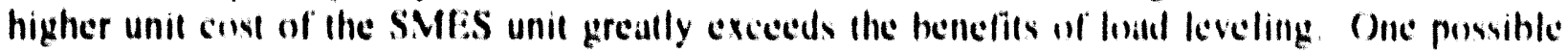

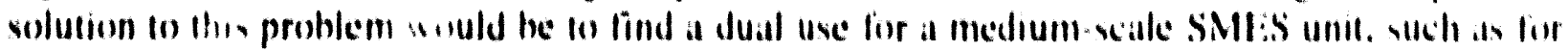
magnetic ore separation and load leveling. To dale, this aption has not been analysed. Anether solution would he to identify an application having a higher value (e g. improving power quality). Hydio Quehec has identified several possible applicallons for a I.(0)(1) MJ SMlis unil and a quadrant.(iTO, 640 MVA PCS Io provide transient stability improvememt, power ascillation damping. and voltage regulation on their transmission system. They have lonnd that such a unit could deliver beller performance than conventional allernallives, but would probahly cow more. A thred possible application for a medium.scale SMISS is heing pursued hy Peiel: They are examining use of SMES Io prevent voltage sags on the BART syolem. The Ilydro (juchere and BART applicutions rely on the ability of the SMISS unit for respond very rapidly (o) changing linad condilluns

So far. all SMES rescaurch hass centered on low-lemperature superconduclurs, such as Nhti and $\mathrm{Nb}_{3} \mathrm{Sn}$ operaling at 4.2 or $1.8 \mathrm{~K}$. However, an HTS-hased SMISS might have several advantages:

- Operating at $77 \mathrm{~K}$ could decrease relrigeration power hy up to a ficelor of o(l as well as reduce the capilal cost assincialed with the refrigeration= and helium. related components.

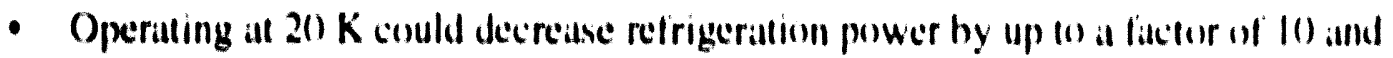
permit design for a higher operating magnetic field.

- An HTS-based SMLSS would be more stable, but should an instability develop. protection of the magnes would be more diflicult. 
- An early application of HTS could be as current leads operating between $77 \mathrm{~K}$ and the operating temperature in an LTS-based SMES. HTS-based current leads could reduce the heat load associated with conventional curtent leads by up to a fictor of 10.

- Major uncertainties remain in regard to how much HTS will cost and what the ultimate level of performance will be.

- Since the conductor represents approximately $20 \%$ of SMES capital cost for all sizes of SMES units. HTS will need to have a cost and performance equal to or better than $\mathrm{NbTi}$ or $\mathrm{Nb}_{3} \mathrm{Sn}$ to have an appreciable impact on SMES cost.

- For a 5.(0)0 MWh SMES, refrigeration- and helium- related capital costs are all most 14\% of total capital costs, and round-trip losses due to refrigeration are only $4 \%$. Furthermore, use of warm structure prohibits going to a higher magnetic field. Therefore, any savings due to operating at a higher (emperature will be incremental unless the cost and performance of HTS is better than that of L.TS.

- For a I MJ/I MW SMES, an analysis by W.J. Schafer and Associates found that unless HTS could be manufactured with a $J_{c}$ grealer than 20.000$) \mathrm{A} / \mathrm{cm}^{2}$ operating in a magnetic field of $4 \mathrm{~T}$ at $77 \mathrm{~K}$ and with a cost less than $\$ 120 / \mathrm{kg}$ (comparable to $\mathrm{Nb}_{3} \mathrm{Sn}$ ), an HTS-based SSD would have a cost comparable (1) simple improvements that could be made to the present SSD system. Over half of the present SSD cost is due to the PCS.

- Another analysis by W.J. Schafer and Associates examined construction of a modular, toroidal, medium-scale SMES unit with a storage capacity ranging between 2 and $200 \mathrm{MWh}$ using HTS operating at 10 T and at 20 K. with an assumed $J_{\mathrm{c}}$ of $20,000 \mathrm{~N} / \mathrm{cm}^{2}$ and a cost of $\$ 100 / \mathrm{kg}$ (comparable to $\mathrm{NbTi}$ ). The HTS-based SMES unit was a factor of two smaller and had refigeration requirements $60 \%$ less than a comparable LTS SMES unit. However, the HTS-based unit cost between a factor of two and three more than the comparable LTS unit depending upon the size of the unit.

- For an HTS-based medium-scale SMES to be cost-effective relative to an LTSbased SMES, Jo values and cost for HTS must be improved 10 values comparable to those for NbTi.

- Since the PCS represents a significant component of cost and inefficiency in all SMES systems, any improvements in the cost and performance of the PCS will improve the cost-effectiveness of the SMES unit. 


\subsection{Flywheel Energy Storage}

Flywheds have heen used to store encrgy for many years. Solid, sted llywheds attached to a motor/gencrator were used during the $196($ s) to smonth out power requirements for synchrotron accelerators. A $f(0)$ MJ thywhel unit was used to smooth wut a 4 -s poiver ramp all the Zero Gradient Synchrotron Accelerator at Argonne Nattonal Lahoratory. Similar devices were used at accelerators at Brookhaven National Laboratory and Lawrence Berkeley Laboratory.

Flywheds have many of the same advantages as an SMES unit:

- The ability to respond to changing loads within one or two cycles.

- No performance degradation alter repealled cycling (long life).

- Little, if any. adverse environmental impact.

In addition. there are no magnetic fields associated with a llywheel, and modular construction of flywheels at the factory could reduce the time and cost associated with on-site construction. Unlike SMES, such a llywhed might be cost-effective for load leveling applications at small sires.

The main disadvantage of tlywheds has heen low round-trip efliciency. A llywheel with conventional mechanical bearings loses approximately $2 \%$ of its stored energy per hour in hearing loss. This would rexult in a round-trip efliciency for diumal. load leveling of approximalely $50 \%$. too low to even consider. The use of magnetic bearings can reduce these losses by approximately one order of magnitude. However. hecause such a system is inherently unstable, some form of active electronic feedback control is required to maintain stability. This increases the complexily and cost of the device.

A superconducting magnetic bearing is stable and it eliminates the need for the active feedback system. A relatively small cryocooler is required to cool the bearing to $77 \mathrm{~K}$. The goal for such a bearing is a net loss. including the ellergy consumed by the eryocooler of $2.4 \% / \mathrm{d}$ $(0.1 \% / \mathrm{h})$. Coupled with an assumed motor/gencrator efficiency of $96 \%$ and an assumed PCS efficiency of $96 \%$, such a llywheel system would have a round-trip efficiency of $90 \%$. comparable with a large-scale SMES unit. Recent experiments at Argonne National Laboratory have demonstrated that this level of loss is achievable in small samples operating at relatively slow speeds. The work is concentrating on scaling up these results to higher operatting speceds and larger bearings.

Work is also proceeding toward improving the efficiency of conventional magnetic bearings. AVCON, a magnetic hearing manufideturer. hats calculatted that improvemeols in 
conventional magnetic bearings could improve their efficiency $2.4 \%$ per day for stationary applications. They are readying an improved magnetic bearing for testing during fall 1993.

- A flywheel system may turn out to be a viable competitor to an SMES system and other conventional storage options. Both an advanced active magnetic bearing and a superconducting magnetic bearing have the potential to reduce stationary losses to approximately $2.4 \%$ per day.

- No cost analyses of such flywheels systems have heen performed for stationary, load-leveling applications. Such a cost analysis needs to be done.

- Whether a superconducting magnetic bearing or an improved, conventional magnetic bearing wins out, will depend upon the relative cost-effectiveness, reliability, and efficiency of each system. Cost analyses remain to be done.

- Because the PCS and the motor/generator set represent significant components of cost and inefficiency in a flywheel system, any improvements in their cost and performance will improve the cost-effectiveness of the flywheel system.

\section{Q.3 Future Directions}

There is still much interest in building a large-scale SMES, particularly in Japan and the United States. Such an SMES unit would provide many benefits including diurnal load leveling, improvement in the dynamic response of generators, and alternative spinning reserve capacity. However, the high cost (in excess of one billion U.S. dollars) of a large-scale SMES unit makes it difficult to obtain adequate funding for such a project. If the present ETM project is terminated, the only large-scale SMES program will be in Japan, where the SMES Research Association is performing a conceptual design study of solenoidal and toroidal coils for an SMES unit with a storage capacity of approximately $20 \mathrm{MWh}$. They have concluded that a solenoidal design is preferred.

Many researchers in the United States, Europe, and Japan are examining the potential for small-scale and medium-scale SMES units. Because these units are significantly less costly than large-scale SMES units, obtaining the necessary funding to design and build such units is easier. Identification of niche markets for small-scale and/or medium-scale SMES units will help maintain interest in SMES techroingy and may spur development of innovative designs for improving SMES performance and reducing SMES cost. Furthermore, as such units are successfully operated in the field, utilities and other potential SMES customers will gain confidence in using superconducting technologies in a nonresearch environment.

The small-scale SMES unit produced by SI illustrates this approach. SI has managed to secure funding from venture capitalists to build the SSD unit. The SSD unit addresses the need to 
improve power quality in a niche market of customers for whom a momentary outage is very expensive. So far, SI has delivered four SSD units to commercial customers and has identified many other potential customers. SI has used the experience gained from operation of each SSD unit to make improvements in subsequent units. Groups in Europe and Japan are also examining the potential market for such units in their respective countries.

Interest in medium-scale SMES units is also grov'ing. In North America, Hydro Quebec is actively investigating use of a 1,000 MJ (278 kWh), 640 MVA SMES unit to improve transient stability, power oscillation damping, and voltage regulation on portions of their high-voltage transmission system. Similar efforts are underway in Japan and Europe. In Japan, several utilities have experimentally verified use of SMES units to provide stabilization and frequency control using small SMES units that have a storage capacity of less than $1 \mathrm{kWh}$. Presently, there is a MITI/ISTEC project to demonstrate a $100 \mathrm{kWh}$ SMES unit. In Europe, a collaboration between Siemens AG, Preussen Elektra AG and VARTA Batterie AG has begun a two-year feasibility project to examine use of a medium-scale SMES unit to provide spinning reserve for about $30 \mathrm{~s}$ while conventional thermal generating units ramp up to meet the increased load. Preliminary calculations indicate that an SMES unit with $2 \mathrm{MWh}$ of energy storage and a power rating of $125 \mathrm{MW}$ would be adequate.

Much progress has been made toward producing practical HTS-based wire and tape. Current efforts are concentrating on manufacturing techniques to produce long lengths of wire and tape and to reduce cost. Presently, efforts are underway to incorporate HTS-based current leads into the SI SSD unit. A likely early application of HTS may be as current leads in other lowtemperature SMES devices. As the performance of HTS increases and the cost decreases, all HTS-based SMES devices may become practical. However, it would be a mistake to wait until HTS-based wire and tape is fully developed to begin building and demonstrating SMES devices. Rather than resulting in revolutionary changes. HTS will more likely result in incremental improvements in the cost and performance of SMES devices, permitting the more widespread use of such devices. Thus, research on low-temperature SMES devices should continue to solve the various engineering and power electronics issues common to both low-temperature and hightemperature SMES devices in parallel with efforts to produce practical HTS-based wire and tape. 


\section{References}

1. Ferrier, M., Stockage d'energie dans un enroulement supraconducteur, Low Temperature and Electric Power, pp. 425-432, Pergamon, London, England (1970).

2. Boom, R.W. and H.A. Peterson, Superconductive Energy Storage for Power Systems, IEEE Transactions on Magnetics, Vol. Mag-8, No. 3, pp. $701-703$ (September 1972).

3. Mohan, N., Superconductive Energy Storage Inductors for Power Systems, Ph.D. dissertation, Electrical and Computer Engineering Deptartment, University of Wisconsin, Madison, Wisconsin (May 1973).

4. Hassenzahl, W.V., B.L. Baker, and W.E.Keller, The Economics of Superconducting Magnetic Energy Storage Systems for Load Leveling: A Comparison with Other Systems, Los Alamos National Laboratory, Report LA-5377-MS (August 1973).

5. Powell, J.R. and P. Bezler, A comparison of Warm- and Cold-Reinforcement Magnet Systems for Tokamak Fusion Reactors, in Proceedings oof Technology of Controlled Thermonuclear Fusion Experiments and the Engineering Aspects of Fusion Reactors, pp. 358-383, Austin, Texas (1973).

6. Boom, R.W., et al., Wisconsin Superconductie Energy Storage Project, Vol I, pp. III:1-5, University of Wisconsin, Madison, Wisconsin (1974).

7. Loyd, R.J., et al., Design Improvements and Cost Reductions for a 5,000 MWh Superconducting Magnetic Energy Storage Plant, Los Alamos National Laboratory, Report LA-10320-MS (October 1984).

8. Eyssa, Y.M., et al., The Potential Impact of Developing High $T_{c}$ Superconductors on Superconductive Magnetic Energy Storage, Advances in Cryogenic Engineering, Vol. 33, p. 69, Plenum Press, New York (1988).

9. Loyd, R.J., et al., Design Improvements and Cost Reductions for a 5,000 MWh Superconducting Magnetic Energy Storage Plant, Part 2, Los Alamos National Laboratory, Report LA-10668-MS (October 1985).

10. Hull, J.R., et al., Design and Fabrication Issues for Small-Scale SMES, Cryogenic Engineering Conference, Huntsville, Alabama (June 11-14, 1991).

11. Roger, J.D., W.V. Hassentahl, and R.I. Schermer, I-cuhdinrnal Load-Leveling Superconducting Magnetic Energy Storage System Reference Design, Los Alamos National Laboratory, Report LA-7885-MS, Vols. I-VIII (1980). 
12. Loyd, R.V., et al., Design Improvements and Cost Reductions for a 5,000 MWh Superconducting Magnetic Energy Storage Plant, EPRI Report EM-347 (April 1984).

13. Tanaka, T., SMES R\&D in Nortin America, Europe and Japan, ISTEC Journal, Vol. 4, No. 4, pp. 35-39 (1991).

14. Business Week (April 8, 1991).

15. Daugherty, M.A., et al., SSD Operating Experience, IEEE Transactions on Applied Superconductivity, Vol. 13, No. 1, pp. 204-206 (March 1993).

16. Emerick, J. and L. Ray, Appplication of the Superconducting Magnetic Energy Storage Device (SSD) at a Plastics Manufacturing Facility, Proceedings of the American Power Conference, Vol. 55, No. 2, pp. 1800-1803, Chicago, Illinois (1993).

17. Schoenung, S.M., et al., Cost Savings and Prospects for Applications of Micro superconducting Magnetic Energy Storage (SMES) Using High Temperature Superconductor, IEEE Transactions on Applied Superconductivity, Vol. 13, No. 1, pp. 200-203 (March 1993).

18. Douglas, J., The Delivery System of the Future, EPRI Journal, pp. 4-11 (October/November 1992).

19. Rogers, J.D., et al., 30-MJ Superconducting Magnetic Energy Storage System for Electric Utility Transmission Stabilization, Proceedings of IEEE, Vol. 71, No. 9, pp. 1099-1107 (September 1983).

20. Gavrilovic, M.M. and G. Bégin, SMES Systems for Transient Stability and Damping Improvement of Power Systems, Proceedings of the American Power Conference, Vol. 55, No. 2, pp. 732-738, Chicago, Illinois (1993).

21. Masuda, M., Industrial Use of Small SMES, Economical and Technological Aspect, Proceedings of International SMES Workshop, KfK, Karlsruhe, Germany (June 19-21, 1991).

22. Eyssam Y.M., X. Huang, and R.W.Boom, Design Considerations for High Temperature (High- $T_{C}$ ) Superconductive Magnetic Energy Storage (SMES) Systems, Cryogenic Engineering Conference, Huntsville, Alabama (June 11-14, 1991).

23. Schoenung, E.M., et al,, Design Aspects of Mid-Size SMES Using High Temperature Superconductors, IEEE Transactions on Applied Superconductivity, Vol. 13, No. 1, pp. 234237 (March 1993). 
24. Earnshaw, S., On the Nature of the Molecular Forces which Regulate the constituition of Luminferous Ether, Transactions of the Cambridge Philisophical Society, Vol. 7, pp. 97-112 (1842).

25. Hellman, E.M., et al., Levitation of a Magnet over a Flat Type II Superconductor. Journal of Applied Physics, Vol. 63, pp. 447-450 (January 1988).

26. Brandt, E.H., Friction in Levitated Superconductors, Applied Physics Letters, Vol. 53, pp. 1154-1556 (October 1988).

27. Weinberger, B.R. and L. Lynds, Low Friction in High Temperature Superconducting Bearings, Applied Physics Letters, Vol. 59, pp. $1132-1134$ (June 1991).

28. Moon, F.C. and P.-Z Chang, High-Speed Rotation of Magnets on High $T_{c}$ Superconducting Bearing, Applied Physics Letters, Vol 56, pp. 397-399 (1990).

29. Takahata, R. and T. Yotsuya, Koyo Engineering Journal, Vol. 138 (1990).

30. Moon, F.C., et al., Levitation Forces, Relaxation and Magnetic Stiffness of Melt-Quenched $\mathrm{YBA}_{2} \mathrm{Cu}_{3} \mathrm{O}_{x}$, Japanese Journal of Applied Physics, Vol. 29, pp. 1257-1258 (July 199()).

31. McMichael, C.K., et al., Practical Adapatation in Bulk superconducting Magnetic Bearing Applications, Applied Physics Letters, Vol. 60, pp. 1893-1895 (April 1992).

32. Takahata, R., H. Ueyama, and A. Kuba, Characterization of Superconducting Magnetic Bearings, Proceedings of 5th. International Symposium of Superconductivity, Kobe, Japan (November 16-19, 1992).

33. Section on Active Magnetic Bearings in Product Catalog for Advanced Controls Technology, Inc. (AVCON).

34. Personnal Communication with Pat McMullely, Advanced Controls Technology, Inc. (AVCON), 5210 Lewis Road, Suit 14, Agoura Hills, California, 91301.

35. Bornemann, H.J., et al., Proceeding of the European Conference on Applied Superconductivity (EUCAS '93), Oct. 4-8, 1992, Göttingen, Germany. 


\section{Appendix A:}

\section{Power Conversion Systems}

A Power Conversion System (PCS) is an integral part of a Superconducting Magnetic Energy Storage System (SMES) or a flywhed incorporating a superconducting magnetic bearing. Consequently, an improvement in the cost and/or performance of a PCS would benefit both SMES and flywheel systems. In the case of an SMES unit, energy is stored in the form of a circulating de current. The PCS converts input ac power to de power, which is used to charge the SMES unit. During discharge, the PCS converts de encrey stored in the SMES coil to ac power.

In the case of a flywhed, a motor/generator converts electrical energy to rotational kinetic energy, which is stored in a rotating flywheel. During charging and discharging of the device, the speed of the flywheel, and consequently the motor/generator, varies. This results in a variable frequency for the motor/generator. The PCS is used to match the frecuency of the motor/generator to the grid frequency.

The following discussion is presented relative to an SMES system. However, many of the issues discussed would also apply to a PCS for a flywheel system with one fundamental difference: the PCS for a SMES converts an ac grid voltage to a constant de voltage, which has some ripple, while the PCS for a flywheel converts an ac grid voltage to controlled de voltage that varies with time to simulate an ac voltage with a frequency lower than the grid frequency. This requires a somewhat more complicated control strategy for generating the gate pulses that control operation of the PCS. In addition, since flywheels operate at speeds up to $33,000 \mathrm{rpm}(550 \mathrm{~Hz})$, a transmission may be required between the tlywheel and the motor/generator so that the motor/generator operates at a frequency less than the grid frequency of 50 or $60 \mathrm{~Hz}$.

For an SMES unit, the voltage $V$ across the superconducting coil is equal to the inductance of the coil L times the time rate of change in coil current $\mathrm{dL} / \mathrm{dt}$. Consequently, the power $\mathrm{P}$ is equal to I $\mathrm{V}=\mathrm{IL} \mathrm{dl} / \mathrm{dt}$. Therefore, to maintain a constant power level during charge and discharge, the voltage across the PCS must vary. The minimum operating current level determines the PCS voltage rating, and the maximum operating current level determines the PCS current rating.

During much of SMES operation, the PCS is underutilized. Furthermore, complete discharge of the SMES would require infinite voltage to maintain a constant $P$. Therefore, either the SMES is only partially discharged or discharge occurs at less than full power. The maximum voltage level is usually limited to $12 \mathrm{kV}$ to prevent excessive turn-to-turn voltages from occurring within the SMES coil. This voltage is consistent with ratings of presently available power electronics components for two modules in series. A smaller voltage might used for a small-scale SMES where a PCS rating of I MVA or less may be all that is required. 


\section{A.1 Diode Bridge}

The simplest SMES charging scheme would be to use high-power diodes in a bridge rectifier together with a transformer. Except for the power level, this scheme is similar to power supplies in conventional electronics, such as VCRs, televisions, and personnel computers. Such diodes bridges were first developed during the 1950 s and are presently available for currents up to $7 \mathrm{kA}$ and voltages up to $6 \mathrm{kV}$. Diodes have very high power ratings, are capable of very rapid switching, have low losses, and are relatively inexpensive.

A diode conducts current whenever the voltage across the diode is positive and blocks current when the voltage is negative. Consequently, this circuit converts the input ac power to a rectified quasi-dc power with a fixed-average de voltage, but provides no voltage control and. consequently, no power control. Furthermore, this circuit does not permit discharge of the SMES unit or conversion of dc to ac.

\section{A.2 Silicon Controlled Rectifler (SCR)}

The simplest form of PCS that provides reversible conversion of ac to de with voltage and power control is a Graetz bridge. Figure A-I illustrates the basic circuit. Instead of a diode, a four-layer thyristor device called a silicon-controlled rectifier (SCR) is used. Like a diode, an SCR requires a positive voltage to conduct current. Unlike a diode, an SCR also requires a positive gate pulse to initiate current conduction. Once conduction begins, an SCR latches in an "on" state until the current is forced to zero and a negative voltage is applied across the SCR. SCRs were first developed during the early 1960s and presently can handle currents up to approximately $3.5 \mathrm{kA}$ and voltages up to approximately $6 \mathrm{kV}$. SCRs have significantly lower (about hall) power ratings, are more expensive, are slightly slower, and have somewhat higher switching losses than diodes. However, in many applications, the added control available in SCRs more than offisets these disadvantages and makes new applications possible.

To facilitate discussion of how a Graetz bridge works, Figure A-2 depicts the circuit for a single phase version of a Graetz bridge. Operation of an SCR is characterized by an angle $\alpha$, which is defined as the phase angle between the positive zero crossing point of the input voltage and the instant that the gate pulse is applied to the SCR. Since a positive voltage is required to initiate conduction in an SCR, alpha can range from zero to $\pi$; for an $\alpha$ of zero, an SCR behaves like a diode. However, by varying $\alpha$, an SCR bridge can control the level of rectified voltage, and consequently the magnitude and direction of power flow. 


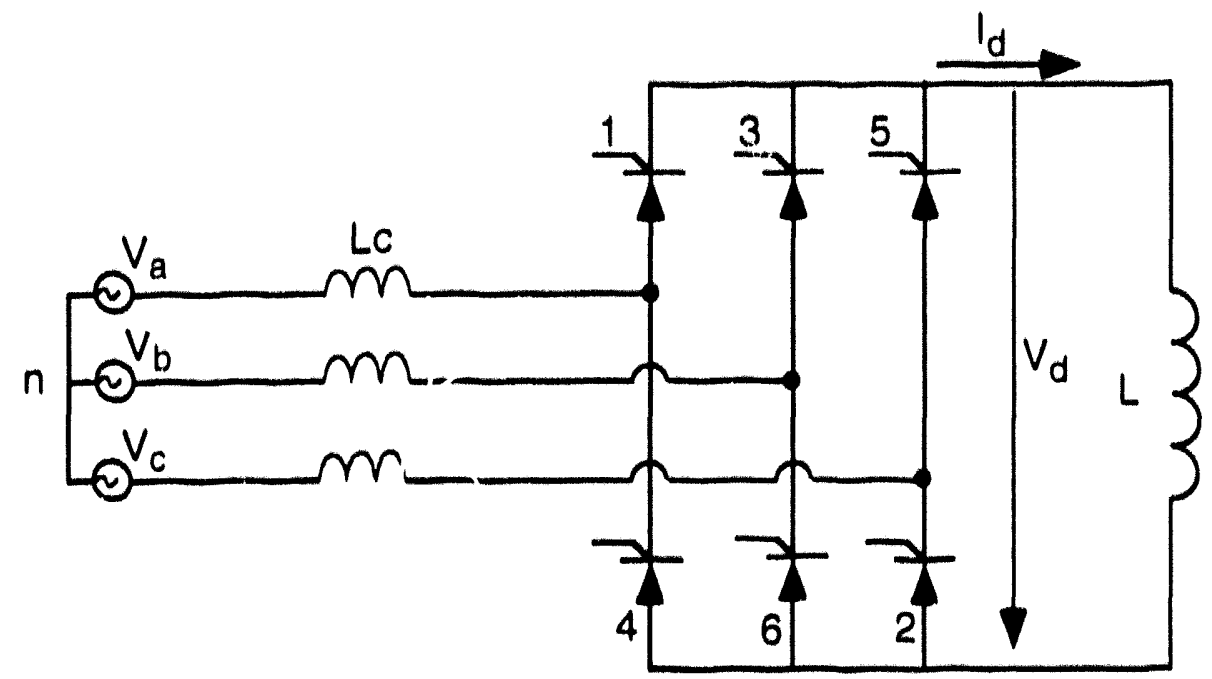

FIGURE A.1 Schematic of a Six-Pulse, Three-Phase Graetz Bridge

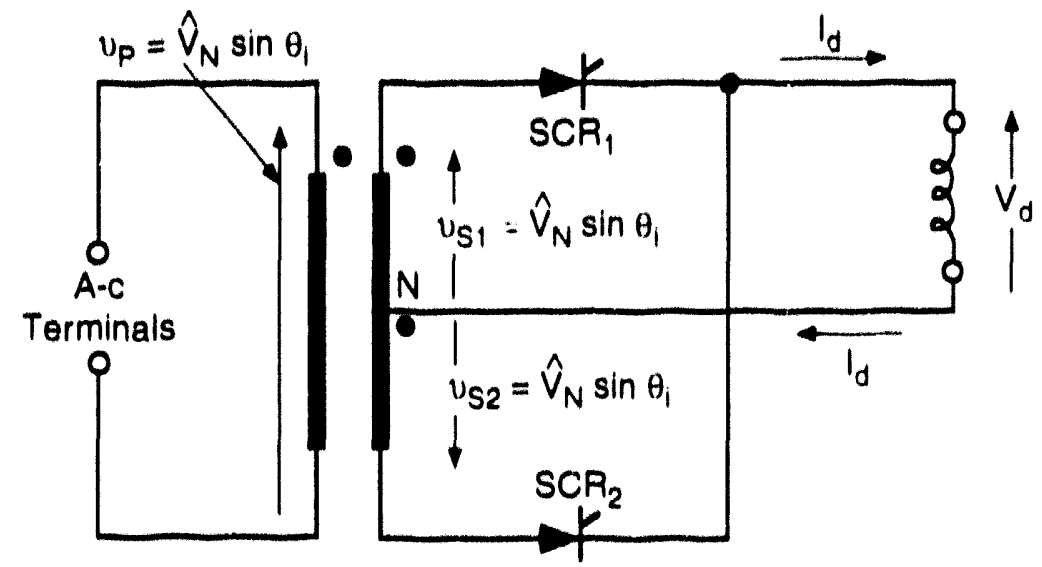

FIGURE A-2 Schematic of Single-Phase Graetz Bridge 
Figure A-3 illustrates the resulting wave forms for several values of $\alpha{ }^{\prime}$ Figure A-3(a) illustrates operation for $\alpha$ equal to zero. The top wave form shows the rectified de terminal voltage. At time $t_{0}, S C R_{1}$ is in the conducting state; there is no voltage across $S C R_{1}$; and the voltage across the de terminals is equal to the input voltage $V_{s 1}$. Meanwhile, $S C R_{2}$ blocks current flow and the full voltage $V_{s 2}$ appears across $S C R_{2}$. Since the SMES unit has a very large inductance, the current flowing into the de load (the SMES unit) is essentially constant during any cycle. This is illustrated in the second wave form.

At time $\iota_{1}, \mathrm{SCR}_{2}$ receives a gate pulse and begins to conduct. This forces the current to commutate from $S_{C R}$ to $S_{2}$. From time $t_{1}$ to time $t_{2}$, the rectified voltage is equal to $\mathrm{V}_{\mathrm{s} 2}$. At

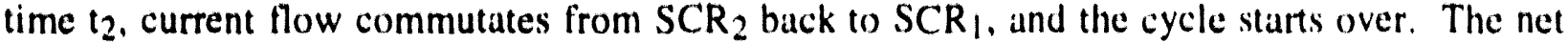
result is that a rectified quasi-de voltage appears across the de terminals of the SMES unit, while a constant charging current flows into the SMES unit. As seen in the third wave form, the supply voltage and supply current are in phase resulting in a power factor approximately equal to one. Thus, the SMES unit absorbs real power and essentially zero reactive power.

Figure A-3(b) illustrates operation of the Graetz bridge for $\alpha$ equal to 45 degrees $(\pi / 4)$. Now conduction in $S C R_{1}$ does not begin until time $t_{()}$. Prior to $t_{()}, S C R R_{1}$ is blocking current flow and $S C R_{2}$ is conducting. The voltage across the de terminals is equal to $V_{S 2}$ (a negative voltage). At $t_{()}$. current flow commutates fiom $S_{2} R_{2}$ to $S_{1} R_{1}$ and the voltage across the de terminals jumps to a positive value. $S C R_{1}$ continues conducting current until $S C R_{2}$ receives a gate pulse at time $t_{1}$, when current flow commutates from SCR 1 back to $S C R_{2}$. The net result is that a lower average rectified quasi-de voltage appears across the SMES terminals and the same constant current flows into the SMES unit. In this case, the supply current lags the supply voltage resulting in a lagging power factor less than one. Thus, the SMES unit absorbs a combination of real and reactive power.

Figure A-3(c) shows operation for an $\alpha$ of $90^{\circ}(\pi / 2)$. In this case, the average rectitied voltage across the SMES unit is zero and the supply current lags the supply voltage by approximately $90^{\circ}$. The net result is that the SMES unit absorbs no real power and a maximum reactive power.

Figure A-3(d) shows operation for $\alpha$ equal to $135^{\circ}(3 \pi / 4)$. In this case, the average rectified voltage across the SMES unit is negative. Since the same current still flows into the SMES unit, the SMES unit now delivers real power while still absorbing reactive power. Thus, by varying the firing angle, a Graetz bridge can reversibly charge and discharge an SMES unit. However, because the supply current always lags the supply voltage, the Graetz bridge always absorbs reactive power. Absorption of reactive power is a maximum for an $\alpha$ of $90^{\circ}$ and minimum

1 For purposes of this illustration, the effects of transformer have been ignored. In a real circuit, the inductance associated with the transformer windings would cause a slight reduction in the de terminal voltage. 
(a)
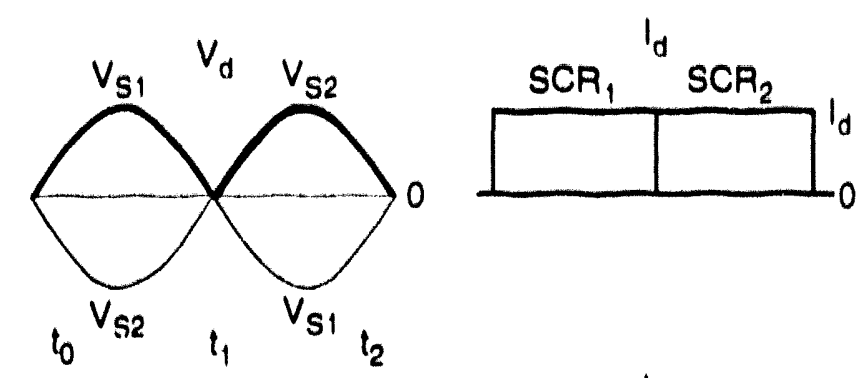

(b)
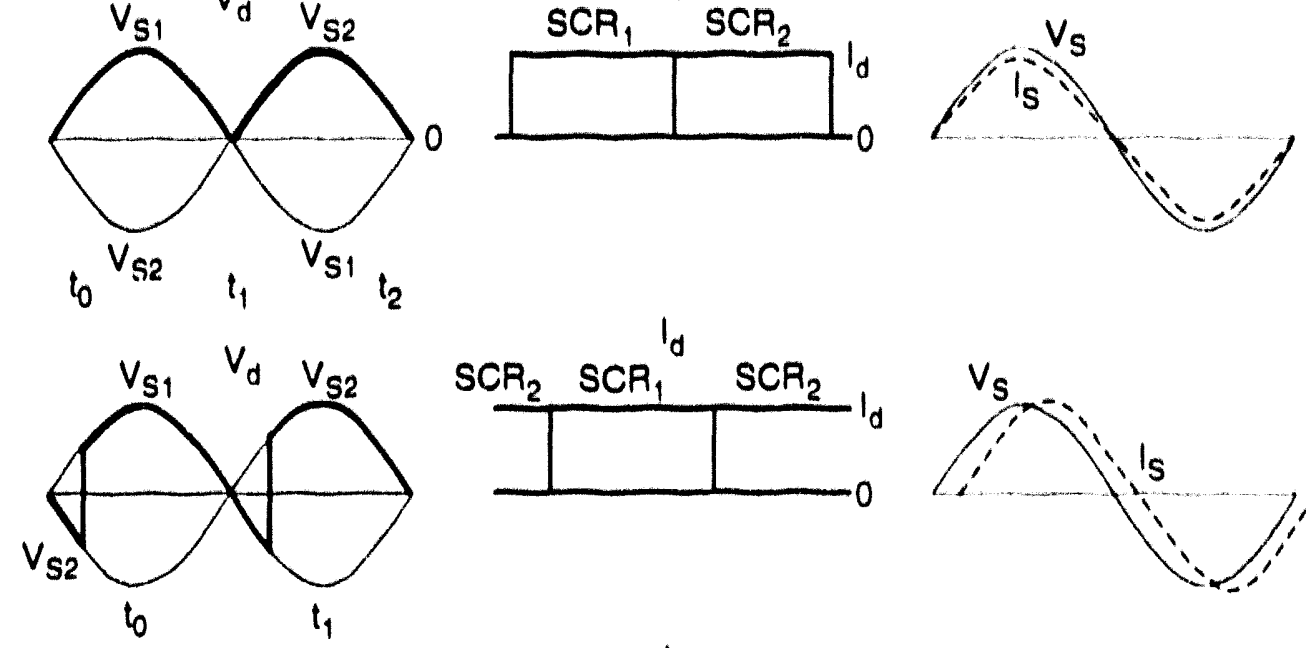

(c)
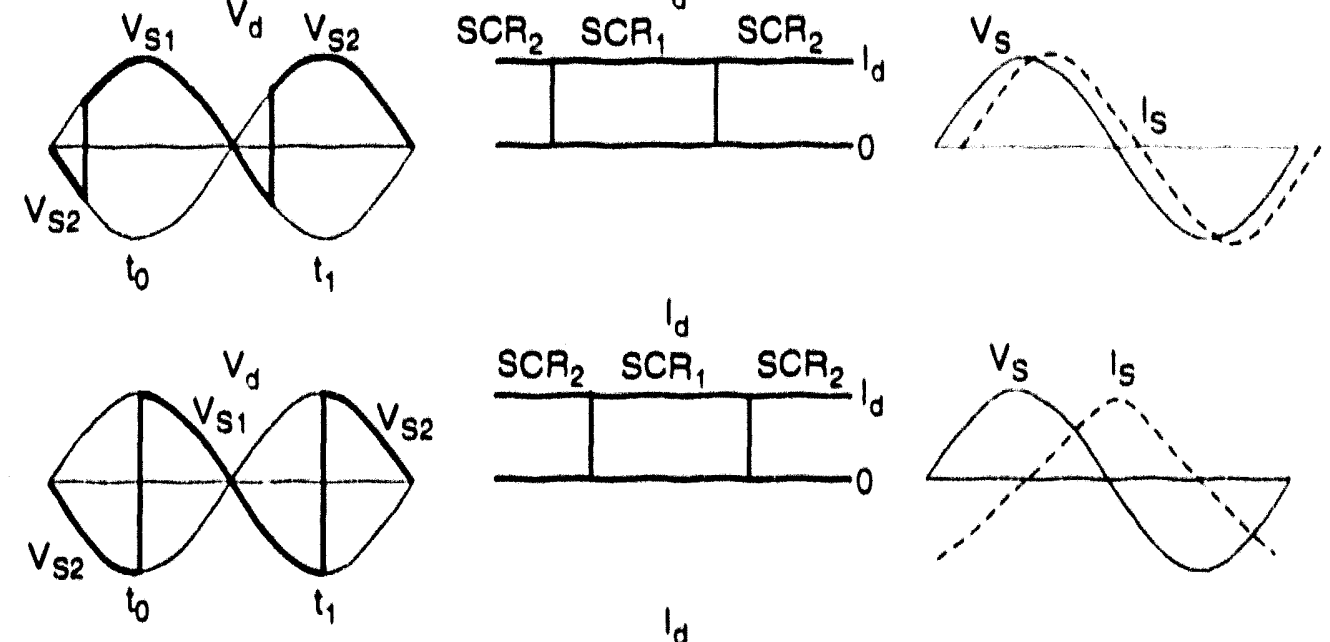

(d)
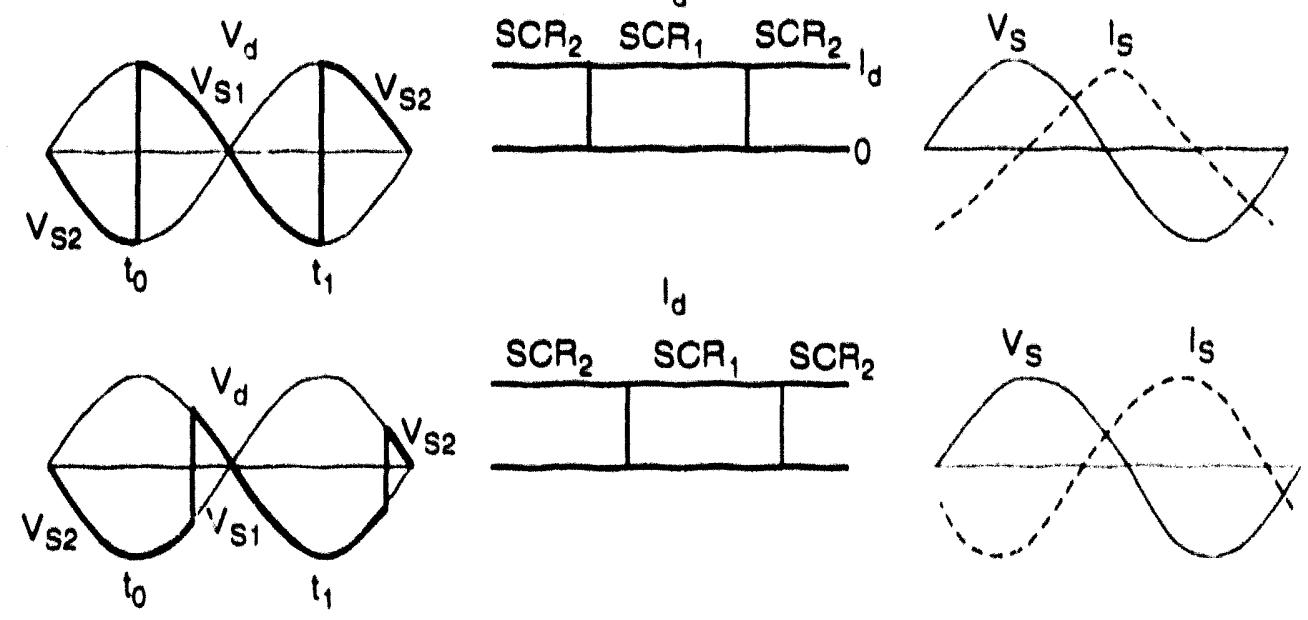

(e)
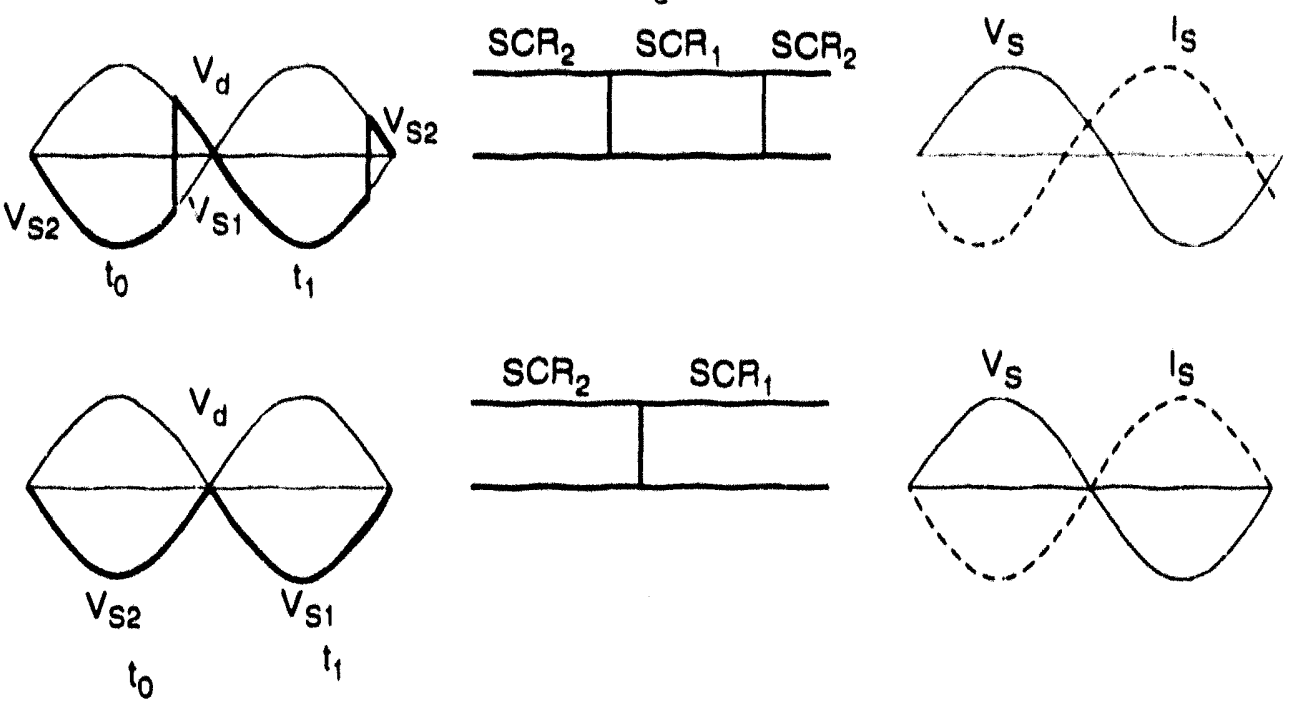

FIGURE A-3 Wave Forms Associated with a Single-Phase Graetz Bridge: (a) for $\alpha=0^{\circ}$, (b) for $\alpha=45^{\circ}$, (c) for $\alpha=90^{\circ}$, (d) for $\alpha=135^{\circ}$, and (e) for $\alpha=180^{\circ}$ 
for $\alpha$ equal $100^{\circ}$ and $180^{\circ}$. Equation $\mathrm{A}-1$ shows how the reactive, Q. and real power, P. flowing into a SMES unit vary with $\alpha$.

$$
\begin{aligned}
& P=P_{11} \cos \alpha \\
& Q=P_{0} \sin \alpha
\end{aligned}
$$

where $P_{0}$ is the power rating of the Graetz bridge. Because reactive and real power are delivered in a fixed ratio determined solely by the value of $\alpha$. consumed by an SCR-hused (iractz hridge the amount of reactive power increases as the PCS operates at a reduced level of real power.

There are several disadvantages associated with a simple Graetz bridge. First, such circuits generate large amounts of harmonics which may require installation of tilter circuits on the input and/or output sides of the bridge. Another method for reducing harmonics is to increase the number of SCRs in the circuit. The simplest three-phase Graetz bridge contains six SCRs and produces six current pulses. Figure A.4 illustrates how using Iwelve SCRs and gencrating Iwelve current pulses produces a wave form which more closely approximates a sine wave. thereby generating fewer harmonics. However, a twelve-pulse Graetz bridge costs more than a six-pulse Graetz bridge. Second, Gruetz bridges absorb significant amounts of reactive power when operating at reduced real power levels. One possible way to reduce consumption of reactive power is to run several Graetz bridges in series and run each bridge with a different $\alpha$. Such a technique can reduce, but not eliminate, reactive power consumption at partial load settings. Third, the amount of reactive and real power can not be independently controlled, and the SMES unit can
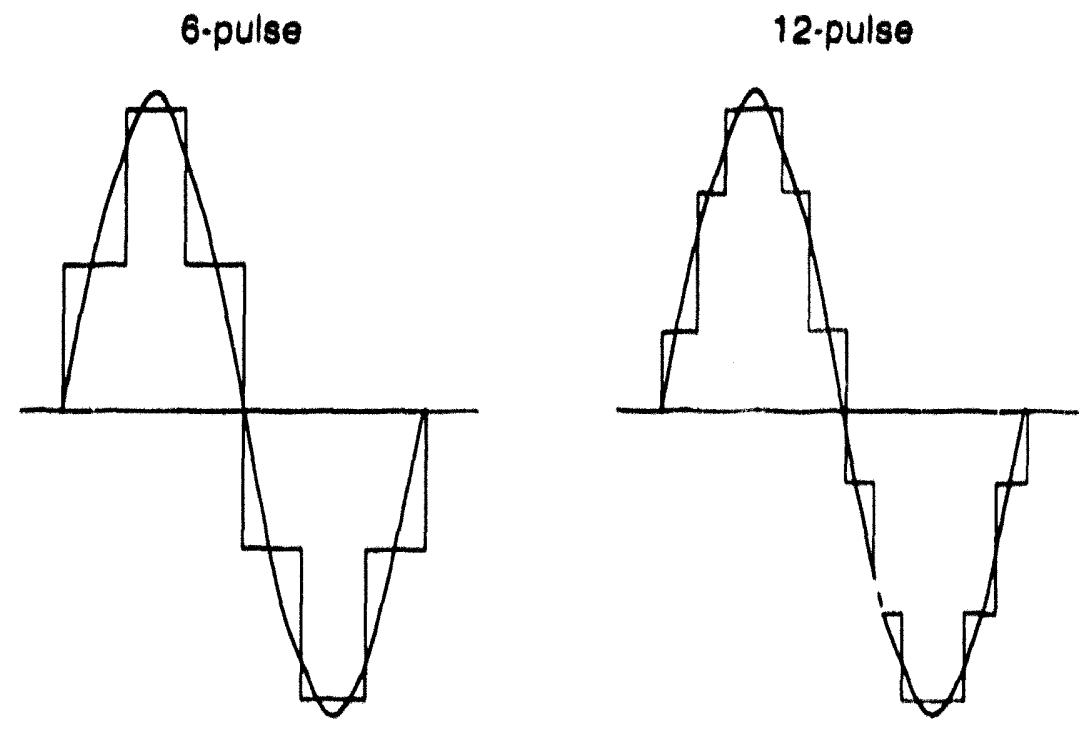

FIGURE A-4 Wave Forms (a) for a Six.Pulse Graetz Bridge, and (b) for a Twelve-Pulse Graetz Bridge 
never be used to generate reactive power. This is because the supply current must always lag the supply voltage in an SCR. To accomplish independent control of reactive and real power, a circuit must either use a Gite-Turn-Off (GTO) thyristor, or use a forced commutation circuil lo cause positive voltage levels atcross the SCR for negative values of $\alpha$.

\section{A.3 Gate-Turn-Off (aTO) Thyrlstor}

A GTO thyristor resembles an SCR, in that it requires a positive gate pulse and a positive biasing voltage to initiate current conduction. Unlike an SCR. current conduction in a CTO can be turned off by application of a negutive gute pulse any time. This permits design of a circuit where the supply current leads the supply voltage (i.e., negutive values of $\alpha$ are possible). GTOs were first developed during the $1980 \mathrm{~s}$ and can presently handle currents up $103 \mathrm{kA}$ and voltages up to $6 \mathrm{kV}$. GTOs have somewhat lower power ratings, slower switching speeds, higher losses, and higher costs than SCRs.

Because the GTO part of the bridge operates with negative $\alpha$ values, the voltage across the GTO at the time of turn may be negative. Therefore. a forced commutation circuit must be employed to drive the voltage positive at the time of application of the positive gate pulse. In addition, a negative gate pulse must be generated to tum off conduction. Typically, an RC snubber circuit is used across the gate to provide the necessary energy 10 accomplish these functions. Because a GTO may be operating at significant power levels at the time of ." 'iching. special precautions must be employed to reduce stray inductance in the snubher cire" 10 prevent excessive voltage from developing during switch off. Although GTO thyristors dite more expensive than SCRs, the forced commutation circuits ussociated with a CTO are simpler, less cosilly, and more compact than would be required for a comparable SCR-based circuit.

By combining a Gruetz bridge consisting only of SCRs in parallel with a Gractz bridge consisting of GTOs, four-quadrant, independent control of both reactive and real power is possible. (If would also be possible to use GTOs in both bridges, but this would he more expensive and entail somewhat higher losses.) A potential disadvantage of this design is that the two bridges operating in purallel have a higher current rating (up to $6 \mathrm{kA}$ ) than a comparable single bridge (up to $3.5 \mathrm{kA}$ ). However, except for small-seale SMES units, the PCS must provide large values of current to the SMES unit. For example, the two ETM designs call for a maximum SMES current of 50 and $250 \mathrm{kA}$, respectively. Thus, many bridges operating in parallel will be required to provide the necessary current, and the use of dual bridges should not be a disadvantage. 
If we assume that the SCR bridge operates at a control angle of $\alpha_{\text {| }}$ while the GTO bridge operates at a control angle of $\alpha_{2}$, then the real power and reactive power are given by:

$$
\begin{aligned}
& P=\frac{P_{0}}{2}\left[\cos \left(\alpha_{2}\right)+\cos \left(\alpha_{2}\right)\right] \\
& Q=\frac{P_{0}}{2}\left[\sin \left(\alpha_{1}\right)+\sin \left(\alpha_{2}\right)\right]
\end{aligned}
$$

where $P_{0}$ is the power rating for the combined SCR/GTO bridges.

If we further assume that $\alpha_{1}$ is muintained equal to $\beta-\alpha_{2}$ where $\beta$ is a fixed angle, then equation 4.2 reduces to:

$$
\begin{aligned}
& P=P_{0} \cos \left(\frac{\beta}{2}\right) \cos \left(\alpha_{2}-\frac{\beta}{2}\right) \\
& Q=P_{0} \sin \left(\frac{\beta}{2}\right) \cos \left(\alpha_{2}-\frac{\beta}{2}\right)
\end{aligned}
$$

For a give real power $P$ and reactive power $Q$. the following relutions cun be used to first determine $\beta$, then $\alpha_{2}$, and finally $\alpha_{1}$ :

$$
\begin{aligned}
\tan \left(\frac{\beta}{2}\right) & =\frac{P}{Q} \\
\cos ^{2}\left(\alpha_{2}-\frac{\beta}{2}\right) & =\frac{P^{2}+Q^{2}}{P_{1}^{2}} \\
\alpha_{1} & =\beta-\alpha_{2}
\end{aligned}
$$

Several years ago, two small experintental devices incorporating a smull SMES and GTObased PCS were built and used to demonstrate independent control of real and reactive power [A-1, A-2]. These units demonstrated that reactive power consumption could be reduced to a very Inw value independent of the level of real power being delivered or absorbed by the PCS and that re 11 and reactive power could be absorbed and generated independently.

\section{A.4 Emerging Devices}

Metal-oxide semiconductors (MOS) integrated circuits which are used in many small electronic devices, such as digital watches, have demonstrated high speed and low energy loss. Work is currently underway to develop a MOS-controlled-thyristor (MCT). In an MCT, MOS 
technology is incorporated into the top layer of a thyristor to reduce losses and increase switching speed. So far. MCTs with a voltage ratting of up $106 \mathrm{kV}$, a current rating of approximately $20 \mathrm{~A}$, and a peak swilching capacily of approximalely $120 \mathrm{~kW}$ have heen huilt. MCT's wilh a calpacily of several MW are expected fo be commercially available by around 1997. New packiging techniques could result in MCTs being more compact and less expensive thatn comparathe (iTO)s. The improved characteristics along with lower cost of MCTs could result in lle ase of MCTs in PCS used for SMES and llywheds incorporating superconducting magnetic hearings. 


\section{A. 10}

\section{References}

A-1 Skiles, J.J., et al., Experimental Experience with a Superconducting Magnetic Energy Storage (SMES) Device On Line, Proceedings of the American Power Conference, Vol. 51, Chicago, Illinois (1989).

A-2. Tominaga, T., et al., Power Control Experiments Using a PWM GTO Thyristor, IEEE/IAS, San Diego, October 1-5 (1989). 

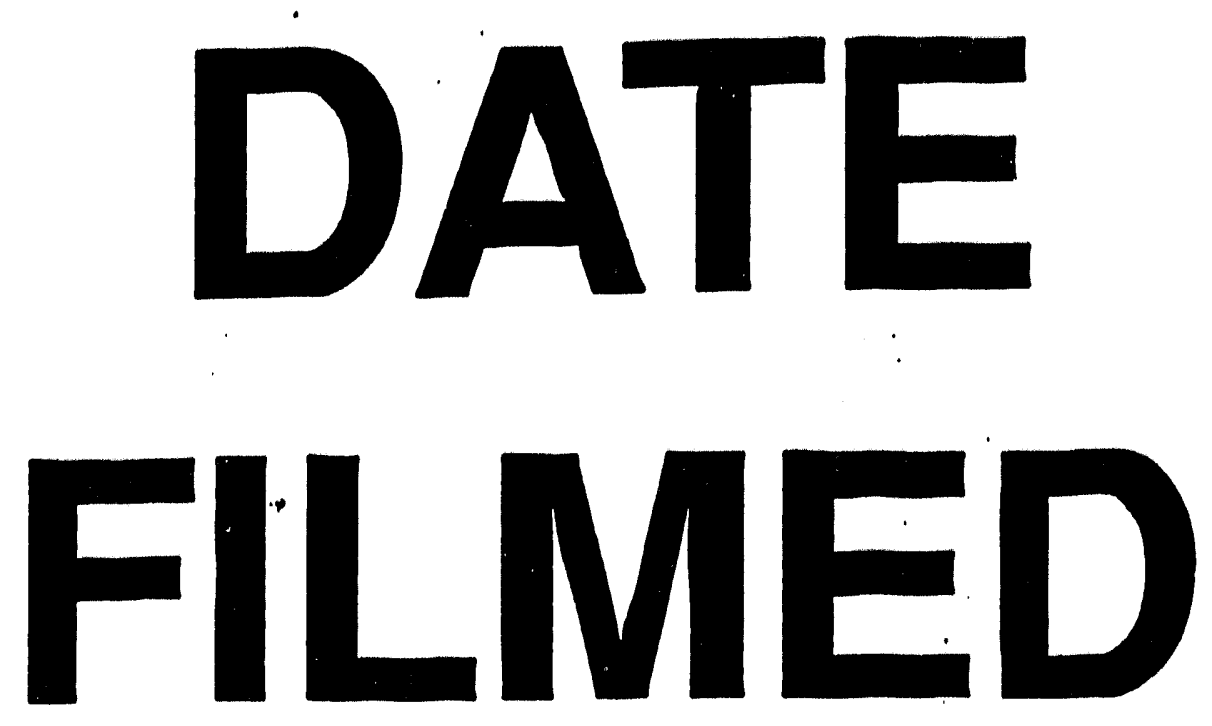

$12 / 27 / 93$
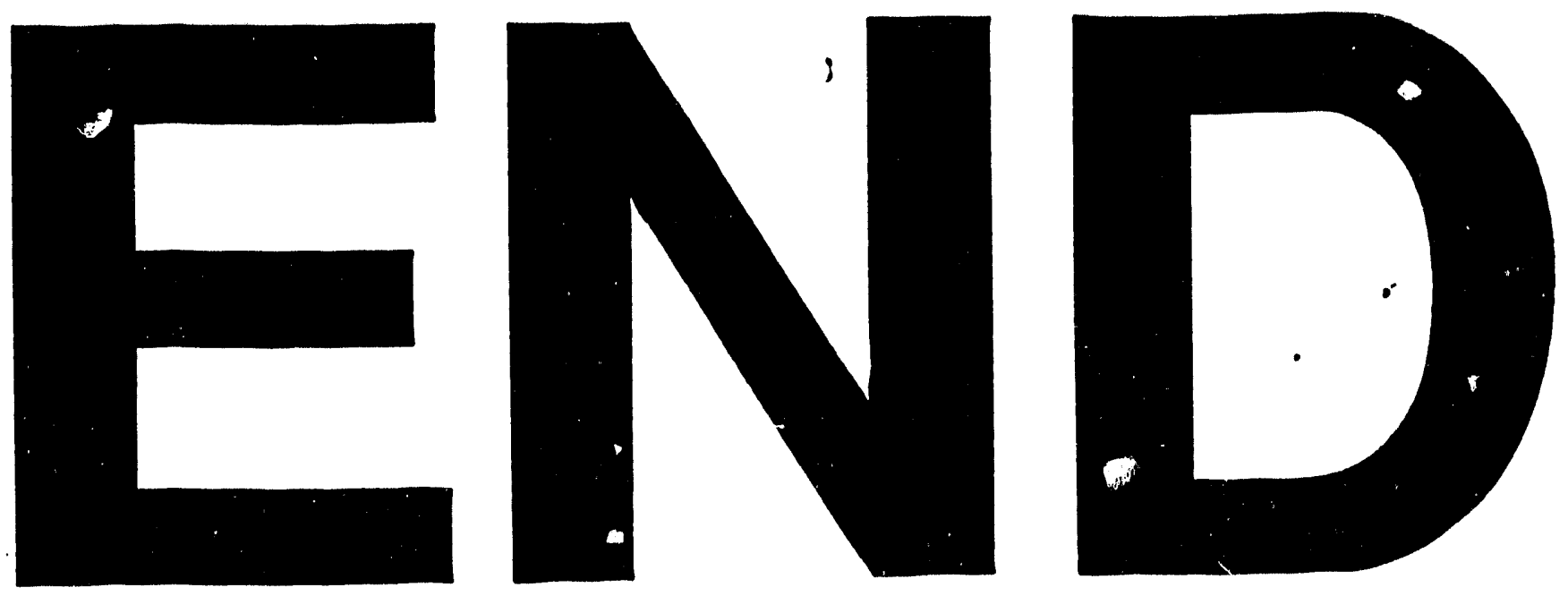
